\title{
Queer Intimacies: A New Paradigm for the Study of Relationship Diversity
}

\author{
Phillip L. Hammack, David M. Frost \& Sam D. Hughes
}

To cite this article: Phillip L. Hammack, David M. Frost \& Sam D. Hughes (2018): Queer Intimacies: A New Paradigm for the Study of Relationship Diversity, The Journal of Sex Research

To link to this article: https://doi.org/10.1080/00224499.2018.1531281

\section{Published online: 26 Oct 2018.}

Submit your article to this journal 지

View Crossmark data $₫$ 


\title{
Queer Intimacies: A New Paradigm for the Study of Relationship Diversity
}

\author{
Phillip L. Hammack \\ Department of Psychology, University of California, Santa Cruz \\ David M. Frost \\ Department of Social Science, University College London \\ Sam D. Hughes \\ Department of Psychology, University of California, Santa Cruz
}

\begin{abstract}
Recognition of sexual and gender diversity in the 21st century challenges normative assumptions of intimacy that privilege heterosexual monogamy and the biological family unit, presume binary cisgender identities, essentialize binary sexual identities, and view sexual or romantic desire as necessary. We propose a queer paradigm to study relationship diversity grounded in seven axioms: intimacy may occur (1) within relationships featuring any combination of cisgender, transgender, or nonbinary identities; (2) with people of multiple gender identities across the life course; (3) in multiple relationships simultaneously with consent; (4) within relationships characterized by consensual asymmetry, power exchange, or role-play; (5) in the absence or limited experience of sexual or romantic desire; (6) in the context of a chosen rather than biological family; and (7) in other possible forms yet unknown. We review research on queer relational forms, including same-sex relationships; relationships in which one or more partners identify as transgender, gender nonbinary, bisexual, pansexual, sexually fluid, "mostly" straight, asexual, or aromantic; polyamory and other forms of consensual nonmonogamy; kink/fetish relationships; and chosen families. We argue that a queer paradigm shifts the dominant scientific conception of relationships away from the confines of normativity toward an embrace of diversity, fluidity, and possibility.
\end{abstract}

As in manufacture, so in science-retooling is an extravagance to be reserved for the occasion that demands it.

$$
\text { —Kuhn (1996, p. 76) }
$$

In the late 19th century in Europe and North America, as the scientific study of sexuality commenced, the ideal form of human intimacy was conceptualized as occurring between individuals of different binary genders (i.e., a man and a woman; Katz, 2007), whose gender identities were presumed to correspond to their sex assigned at birth (i.e., cisgender

Correspondence should be addressed to Phillip L. Hammack, Department of Psychology, University of California, 1156 High Street, Santa Cruz, CA 95064. E-mail: hammack@ucsc.edu individuals). Same-sex ${ }^{1}$ attraction was viewed as a form of gender "inversion" - an intimate interest that violated a gender binary imagined as central to human nature (e.g., Ellis, 1925). A new type or "species" of person was invented - the "homosexual" - whose diversion from presumed notions of the ideal, codified in the cultural and scientific concept of

\footnotetext{
${ }^{1}$ Consistent with current use in cultural and scientific discourse, we use the term same-sex to refer to attraction or intimate relationships between binary cisgender individuals (i.e., individuals whose current binary gender identity matches their sex assigned at birth) who share the same sex. We recognize that some transgender binary individuals may understand and label their relationships as same-sex, but we discuss these relationships separately in the article because research suggests that these relationships are distinct from those in which both partners identify as cisgender (e.g., Pfeffer, 2017).
} 
normativity, resulted in the derogatory label "queer" (e.g., Butler, 1990; Foucault, 1978; Sedgwick, 1990).

Late-19th-century conceptions of heterosexuality and homosexuality reveal the way in which early scientific paradigms in Europe and North America were tainted by cultural ideologies that maintained hierarchies based on factors such as sex, gender, and race (e.g., Gould, 1996; Ordover, 2003). By creating a concept of normativity that not only described but also prescribed ideals of human development, some scientific fields such as psychology and biology supported existing cultural ideologies that privileged particular forms of intimacy (e.g., asymmetric heterosexual relations; Mohr, 2010). Scientists have since worked to challenge these underlying ideologies with respect to gender and sexuality (e.g., van Anders, 2015), and our aim is to offer a novel contribution to such efforts by proposing a queer paradigm that challenges normative assumptions of human intimacy.

In the 21 st century, normative conceptions of human intimacy have been challenged by the liberty and creativity revealed not just in same-sex relationships but also in other diverse and increasingly visible forms, such as polyamory (e.g., Hardy \& Easton, 2017), kink/fetish relationships (e.g., Ortmann \& Sprott, 2013), and relationships in which one or more individuals identify as transgender (e.g., Pfeffer, 2017). Yet the study of relationships has typically continued to depict the nature of intimacy in universalistic terms often concerned with charting and documenting (and thus reifying and reinscribing) (hetero)normative standards (e.g., Finkel, Simpson, \& Eastwick, 2017). As individuals increasingly challenge or reject received taxonomies of sexual and gender identity and dominant standards of normativity in relationships, we suggest the present is an occasion that demands the "retooling" philosopher of science Thomas Kuhn (1996) described in his canonical treatise on the nature of scientific revolutions.

The purpose of this article is to outline a queer paradigm for the study of relationship diversity in the 21 st century that challenges underlying assumptions about the nature and meaning of human intimacy. Our aim is to produce a paradigmatic statement to orient future empirical work and an initial attempt to synthesize knowledge of diverse relational forms that defy normative configurations of intimacy. We begin by outlining seven axioms of a queer paradigm that challenge normative assumptions. We then review and synthesize existing knowledge on the queer relational forms these axioms recognize. We argue that a queer paradigm better captures the diversity, fluidity, and possibility of human intimacy than the normative paradigm that has historically guided relationship science. A queer paradigm thus elucidates vital new knowledge that was once obscured through the lens of the normative paradigm, and it challenges us to rethink the meaning of intimacy more broadly.

\section{A Queer Paradigm}

Following Kuhn (1996), we view a scientific paradigm as the framework or worldview from which scientists operate as they produce knowledge. In contrast to the dominant scientific paradigm of the 20th century, which denigrated diverse forms of intimacy (see Herek, 2010), a queer paradigm takes as its mission the documentation of how individuals defy normative notions of intimacy as they express diversity and creativity in relationships. By "normative," we mean notions conceived as broadly common or standard across a population and framed as prescriptive or ideal (Normative, 2018). By "queer," we mean notions that challenge or deviate enough from the normative to historically warrant social or legal condemnation and/or political opposition. Here we honor the history of the term queer, which emerged in the late 19th century to describe same-sex intimacy that violated law and social prescription (e.g., Chauncey, 1994; Foldy, 1997). A century later, the term was reclaimed with an affirmative usage that views notions of normativity and the "naturalness" of gender and sexual categories as suspect and recognizes the value of queer identities and practices to critique the status quo (e.g., de Lauretis, 1991; Hostetler \& Herdt, 1998; Seidman, 1996; Warner, 1999).

A queer paradigm challenges the historic hegemony of normativity in the study of human relationships. The establishment of norms that not only describe but also historically prescribe relational forms denigrates diversity by delegitimizing that which is nonnormative and establishing hierarchies among forms of intimacy (Rubin, 2011c; Warner, 1999). Rubin (2011c) described a "charmed circle" of privileged forms of intimacy that includes heterosexual, married, monogamous, and procreative sex among individuals of the same generation. Forms of intimacy that fall outside of this circle create hierarchies of shame and include homosexual, unmarried, nonprocreative, cross-generational, and kinky sex (Warner, 1999). The social value placed on particular forms of intimacy creates a context of social and psychological injustice, whereby particular forms are unsanctioned, unrecognized, denigrated, or devalued (Evans, 2007; Plummer, 2003). To the extent that individuals internalize dominant narratives that privilege some forms of intimacy over others, they place themselves at risk for social and psychological challenges and health inequities (Frost \& Meyer, 2009; Hatzenbuehler, 2009, 2014; Meyer, 2003; Szymanski \& Mikorski, 2016).

A queer paradigm is anchored in seven axioms that challenge key assumptions prescribed by normative conceptions of human intimacy (see Table 1). These axioms represent postulates intended to guide new empirical inquiry on relationship diversity. First, a queer paradigm challenges the normative privileging of different binary cisgender attraction in intimacy. Since the late 19th century, the binary cisgender male-female (i.e., heterosexual) 
Table 1. Normative Assumptions versus Queer Axioms of Intimacy

\begin{tabular}{|c|c|c|c|c|}
\hline & $\begin{array}{l}\text { Normative } \\
\text { Assumption }\end{array}$ & Description & $\begin{array}{l}\text { Queer } \\
\text { Axiom }\end{array}$ & Description \\
\hline 1 & $\begin{array}{l}\text { Different binary } \\
\text { cisgender }\end{array}$ & $\begin{array}{l}\text { Intimacy occurs among individuals of different binary } \\
\text { cisgender identities (i.e., male/female) }\end{array}$ & $\begin{array}{l}\text { Same binary } \\
\text { cisgender, } \\
\text { nonbinary, and } \\
\text { transgender }\end{array}$ & $\begin{array}{l}\text { Intimacy may occur among individuals of any gender } \\
\text { identity (i.e., male/female, male/male, female/ } \\
\text { female), including transgender binary and nonbinary } \\
\text { identities (e.g., cis man/trans man; nonbinary/cis } \\
\text { woman) }\end{array}$ \\
\hline 2 & $\begin{array}{l}\text { Singularity and } \\
\text { staticity } \\
\text { across the life } \\
\text { course }\end{array}$ & $\begin{array}{l}\text { Intimacy is directed in a manner that is singular (i.e., } \\
\text { monosexual) and static across the life course (e.g., } \\
\text { attraction to a different binary gender endures and } \\
\text { reflects a static heterosexual identity) }\end{array}$ & $\begin{array}{l}\text { Plurality and } \\
\text { fluidity across } \\
\text { the life course }\end{array}$ & $\begin{array}{l}\text { Individuals may pursue intimacy with different, same, } \\
\text { or no binary gender identities (e.g., plurisexual, } \\
\text { bisexual, or pansexual; heteroflexible or mostly } \\
\text { straight); intimacy may change across the life course, } \\
\text { with changes in sexual identity labels, desires, and } \\
\text { interests }\end{array}$ \\
\hline 3 & $\begin{array}{l}\text { Monoamory and } \\
\text { dyadic } \\
\text { monogamy }\end{array}$ & Intimacy occurs between two individuals only & $\begin{array}{l}\text { Polyamory and } \\
\text { consensual } \\
\text { nonmonogamy }\end{array}$ & Intimacy occurs among multiple partners with consent \\
\hline 4 & $\begin{array}{l}\text { Role symmetry/ } \\
\text { equality }\end{array}$ & $\begin{array}{l}\text { Intimacy is characterized by equality and symmetry of } \\
\text { role and status }\end{array}$ & $\begin{array}{l}\text { Kink/fetish/ } \\
\text { BDSM }\end{array}$ & $\begin{array}{l}\text { Intimacy is characterized by consensual asymmetry } \\
\text { based on power exchange }\end{array}$ \\
\hline 5 & $\begin{array}{l}\text { Sexual and } \\
\text { romantic }\end{array}$ & $\begin{array}{l}\text { Intimacy is characterized by romance and sexual } \\
\text { activity }\end{array}$ & $\begin{array}{l}\text { Asexual/ } \\
\text { aromantic }\end{array}$ & $\begin{array}{l}\text { Intimacy may occur in the absence or limited } \\
\text { experience of sexual or romantic desire }\end{array}$ \\
\hline 6 & $\begin{array}{l}\text { Biological } \\
\text { family }\end{array}$ & Family structure consisting of biological offspring & Chosen family & $\begin{array}{l}\text { Family structure defined by identity and community } \\
\text { connection }\end{array}$ \\
\hline 7 & $\begin{array}{l}\text { Essential } \\
\quad \text { intelligibility }\end{array}$ & $\begin{array}{l}\text { Essential, timeless forms of intimacy can be known and } \\
\text { cataloged }\end{array}$ & Open possibility & $\begin{array}{l}\text { Forms of intimacy are always historically and culturally } \\
\text { situated and in states of constant contestation and } \\
\text { creative emergence }\end{array}$ \\
\hline
\end{tabular}

configuration has been idealized as the ultimate relational form in terms of cultural meaning and value (Blank, 2012; Katz, 2007). A queer paradigm challenges this idealization and recognizes that intimate relations may occur between individuals of the same binary cisgender identity (e.g., male-male, female-female), as well as between individuals who do not conform to a binary cisgender identity. In addition, a queer paradigm recognizes that forms of intimacy may occur in the context of a relationship in which one or more individuals identify as transgender. A queer paradigm recognizes that unique experiences may exist for these distinct types of configurations. In this article, we refer to these queer relational forms as same-sex intimacies to describe the former (i.e., two individuals of the same binary cisgender identity) and transgender intimacies to describe the latter (i.e., two individuals, one or both of whom may identify as transgender or gender nonbinary).

Second, a queer paradigm challenges the normative notion of static and singular desire across the life course. The normative assumption is that intimacy is most commonly directed toward a singular binary gender and that this form of desire, increasingly called monosexual (e.g., Galupo, Mitchell, \& Davis, 2015), remains static across the life course. For example, individuals are presumed to be either same-sex or different-sex attracted (e.g., gay, lesbian, straight) and to maintain a commitment to these types of relational forms across the life course (see DeLamater \& Hyde, 1998; Hammack, 2005). A queer paradigm rejects this essentialist, categorical view of sexual orientation and recognizes that many individuals have the capacity for sexual desire to both binary genders (i.e., individuals who identify as bisexual; e.g., Rust, 2002; Weinberg, Williams, \& Pryor, 1994) or desire that views gender beyond binaries (i.e., individuals who identify as pansexual; e.g., Callis, 2014). Such individuals are increasingly referred to as plurisexual (e.g., Galupo et al., 2015). A queer paradigm also acknowledges that individuals who identify totally, primarily, or mostly with a binary sexual identity label (e.g., straight, gay) may pursue intimacy that defies the normative meaning associated with a categorical sexual identity. For example, the growing number of individuals who identify as "mostly straight" engage in relational forms we argue are "queer" in the sense of defying normative prescriptions about the singularity of sexual desire (e.g., Carrillo \& Hoffman, 2018). A queer paradigm rejects a static view of intimate desire, recognizing that fluidity in sexual and/or romantic desire is possible across the life course (e.g., Diamond, 2008; Katz-Wise, 2015; SavinWilliams, 2017).

Third, a queer paradigm challenges the normative privileging of monoamory and dyadic monogamy in intimacy (e.g., Manley, Diamond, \& van Anders, 2015). The normative assumption is that intimacy occurs between two individuals who restrict their sexual and romantic lives to each other (Emens, 2004; Hidalgo, Barber, \& Hunter, 2008). The idealization of this configuration and its codification through social institutions such as marriage has an extensive history (e.g., Barash \& Lipton, 2001; Coontz, 2005; Seidman, 2015). A queer paradigm challenges the notion that monoamory and dyadic monogamy represent a single 
ideal form of intimacy and posits that meaningful forms of intimacy may occur among more than two individuals. In this article, we refer to these queer relational forms as falling within various configurations of consensual nonmonogamy, including swinging, open relationships, and polyamory.

Fourth, a queer paradigm challenges the normative privileging of intimacy framed by power symmetry and the absence of role or other forms of power play. The normative notion of role symmetry within intimate relations is actually relatively new and not as universally accepted as other norms, given the historic and contemporary subordination of women in heterosexual relations (Botkin, Weeks, \& Morris, 2000; Coontz, 2005). A queer paradigm recognizes consensual forms of asymmetric relations and forms of intimacy in which role play and power exchange are central (e.g., Ortmann \& Sprott, 2013; Weinberg, 2006). We refer to these queer relational forms as kink/fetish/BDSM ${ }^{2}$ and inclusive of many explicitly defined role-based asymmetric relations.

Fifth, a queer paradigm challenges the normative assumption that intimacy is predicated on sexual and/or romantic attraction. We recognize that meaningful forms of intimacy occur among individuals who identify as asexual, aromantic, or with some label associated with the asexual or aromantic spectrum (e.g., demisexual, graysexual; see Bogaert, 2012). A queer paradigm recognizes that these forms of intimacy are likely unique and merit both cultural recognition and empirical study.

Sixth, a queer paradigm challenges the normative conception of the biological nuclear family as the primary social unit in the lives of individuals. The normative assumption of the family concept is that it is rooted in biological relations, typically headed by two individuals (historically of different binary gender identities), and composed of biological offspring (Weston, 1991). A queer paradigm recognizes the concept of the chosen family as an alternative social unit that warrants recognition and empirical study.

Finally, a queer paradigm challenges the normative assumption that human intimacy is characterized by essential intelligibility - that essential, timeless forms of intimacy can be known or cataloged. A queer paradigm posits an axiom of open possibility - that forms of intimacy are always historically and culturally situated and in states of constant contestation and creative emergence (e.g., Foucault, 1978). Thus, a queer paradigm remains constantly open to creativity and innovation in human intimacy, balancing the need for representation of diversity with resistance to reification. In other words, a queer paradigm calls for the documentation of relational diversity in historical and cultural context but simultaneously rejects any attempt to "normalize" relational forms, as a queer stance necessarily entails a rejection of normativity in any form (e.g., Warner, 1999).

\footnotetext{
${ }^{2}$ BDSM is a compound acronym derived from the terms bondage and discipline, dominance and submission, and sadism and masochism (Ortmann \& Sprott, 2013).
}

In sum, a queer paradigm challenges normative configurations of intimacy as restricted to two individuals of symmetrical roles but different binary gender identities that coincide with sex assigned at birth, experiencing desire in a categorical and static manner across the life course, intrinsically characterized by sexual and romantic desire, resulting in a family unit defined by biological relation, and subject to essential intelligibility through taxonomic specificity. Queer relational forms deviate from this normative configuration, and to our knowledge ours is the first attempt to synthesize scientific knowledge across such forms. Similar to challenges of the normative conception of sexual orientation as a unidimensional construct (e.g., van Anders, 2015), our queer intimacies paradigm seeks to expand understandings of diversity in intimacy beyond the gender or sex of attraction. By upending normative assumptions of intimacy, a queer paradigm goes beyond description of diverse relational forms. With a radically divergent set of axioms that counters the normative assumptions that have historically (mis)guided our understanding of relationships, a queer paradigm provides an entirely new lens through which to consider the meaning and diversity of human connection.

We recognize that many queer relational forms existed prior to the 21 st century. For example, there is historical evidence to suggest that individuals of the same binary gender identity have long formed intimate bonds (e.g., Boswell, 1994) and that monogamy is not a timeless standard for relationships (e.g., Barash \& Lipton, 2001). We suggest, however, that new vocabularies and taxonomies have emerged that now give these relational forms a contemporary meaning. Thus, we focus in our review on contemporary manifestations of queer intimacy, recognizing the open possibilities of the emergence of new, unknown queer relational forms. We suggest that scholars embrace a paradigm of queer intimacies that recognizes diversity, creativity, and expansion of existing meanings and structures.

\section{Focus and Parameters}

Our selection of particular queer relational forms on which to focus was guided by our identification of the key normative assumptions about sex, gender, and the family just outlined. While we recognize that other relational forms exist that challenge aspects of normative intimacy beyond sex, gender, and the family (e.g., interracial relationships, interfaith relationships, intergenerational relationships), these other diverse relational forms are beyond the scope of our paradigm. As both scholars and practitioners of various queer relational forms ourselves (e.g., same-sex, polyamorous, kink/fetish/BDSM, chosen families), we recognized the need for a paradigmatic statement and a narrative review of existing literature in this area. We employed the method of a narrative review because our intent was to identify and synthesize a broad, emerging concept (queer relational forms that challenge normative 
assumptions about intimate relationships), rather than answer a narrowly defined research question, which would call for a systematic protocol (e.g., Bettany-Saltikov, 2010).

Guided by the seven axioms of a queer paradigm outlined, we identified seven categories of queer relational forms: (1) same-sex intimacies; (2) transgender and gender-nonbinary intimacies; (3) plurisexual (i.e., bisexual, pansexual, and sexually fluid) intimacies; (4) polyamory and other consensual nonmonogamies; (5) kink/fetish/BDSM intimacies; (6) asexual and aromantic intimacies; and (7) chosen families. Consistent with our seventh axiom of open possibility, we do not claim that these seven forms are exhaustive of contemporary expressions of queer intimacy. Rather, we highlight these forms to reveal the necessity and vitality of a queer paradigm that challenges normative assumptions of intimacy. We collected published, peer-reviewed, English-language research using three major databases (i.e., PsycINFO, EBSCOhost Academic Search Complete, and Google Scholar) and also scanned the reference lists of these research reports for relevant studies that warranted inclusion in our review, using these seven queer relational forms as a guide to determine inclusion. Importantly, articles that focused on queer individuals, instead of queer relationships, were not included in this review.

A queer paradigm has universal relevance in its interrogation of normative assumptions of intimacy. Any application, however, occurs in a particular cultural context, and we recognize the considerable variability in the meaning of gender, sexuality, and relationships across cultures (e.g., Blackwood, 2000; Parker, 2009). For example, normative assumptions of monoamory and dyadic monogamy may not extend to many societies that value plural relationships within communities (see Barash \& Lipton, 2001). Hence, the axioms of a queer paradigm are historically and culturally contingent. Ours is thus not intended as an exhaustive review of relationship diversity across cultures. Instead, the nature and significance of queer intimacies we explore here are generally limited to North American and European societies in which the normative assumptions we outlined have been constructed and deployed. The application of a queer paradigm to other cultural contexts would be extraordinarily beneficial to the identification of patterns of commonality and difference across contexts. Because our aim was to identify the nature of queer relational forms as currently documented and understood within the scientific community, we chose to review only published, peer-reviewed work, which itself tends to be narrowly focused on North American and European contexts. The inclusion of unpublished work in future reviews is recommended, especially for meta-analyses of quantitative studies.

\section{Same-Sex Intimacies}

The first axiom of a queer paradigm for the study of relationship diversity is that intimacy need not be confined to individuals of a different binary cisgender identity. Rather, intimacy may be experienced in the context of a relationship between individuals of any gender identity, including individuals of the same binary cisgender identity, a binary transgender identity, or a gender-nonbinary identity. In this section, we review empirical research on relationships between individuals of the same cisgender identity.

Empirical work on same-sex relationships began to emerge as the American Psychiatric Association removed homosexuality from the Diagnostic and Statistical Manual of Mental Disorders (DSM) in 1973 (American Psychiatric Association, 1973). After this momentous decision, empirical attention gradually shifted away from a focus on samesex attraction as a form of psychopathology and more on the unique psychological experience of same-sex-attracted individuals as sexual minorities in a cultural context of heterosexism and sexual prejudice (Herek, 1990). In the scientific literature, same-sex attraction thus came to be seen as a trait associated with diversity in intimate desire rather than an indicator of pathology (Hammack, Mayers, \& Windell, 2013; Herek, 2010).

The American Couples Study (Blumstein \& Schwartz, 1983) was the first large-scale national study of couples to include a sizable and analyzable sample of same-sex couples, even though a focus on same-sex couples was not the primary aim of the study. Additional empirical investigations into same-sex couples emerged within psychology (for historical reviews, see Kurdek, 2005; Peplau \& Fingerhut, 2007). This research followed two primary strands, which continue to characterize the current state of the field of research on same-sex couples: (a) comparative studies examining the degree to which same-sex couples are similar to and different from different-sex couples and (b) within-group studies on the unique aspects of same-sex couples that are not shared by different-sex couples, typically because of gender dynamics and/or social stigma. Comparative research has been framed as problematic by some operating within critical and queer perspectives because such studies often frame different-sex couples as the "norm" to which same-sex relationships are compared (e.g., Kitzinger \& Wilkinson, 2004). While we acknowledge the legitimacy of this perspective, we believe there is value in reviewing this comparative work to inform an emerging queer paradigm that considers the ways in which contemporary same-sex relationships navigate normativity.

\section{Similarities and Differences Between Same-Sex and Different-Sex Relationships}

Studies comparing same-sex and different-sex relationships have found very few differences between the two using a multitude of indicators of relationship quality, including satisfaction, love, commitment, conflict, and communication (e.g., Balsam, Beauchaine, Rothblum, \& Solomon, 2008; Diamond, 2006; Kurdek, 2005; Peplau \& Fingerhut, 2007), and some studies suggest that same-sex relationships evidence 
more positive functionality than different-sex couples (e.g., Kurdek, 2004). Although early research found higher rates of dissolution among same-sex couples (Blumstein \& Schwartz, 1983), such comparisons were primarily made in relation to different-sex married couples, which presents a confound for comparison (i.e., availability of legal recognition) that has plagued much of the comparative work on same-sex and different-sex relationships. Indeed, when more appropriate comparisons are made controlling for legal and parenting status - both major factors in relationship dissolution-similar levels of relationship dissolution are observed between same-sex and different-sex couples (for reviews, see Diamond, 2006; van Eeden-Moorefield, Martell, Williams, \& Preston, 2011). Even further, the processes that explain relationship commitment-namely investment, satisfaction, and the perceived quality of alternatives to the relationshipappear to be similar across both same-sex and different-sex relationships (Beals, Impett, \& Peplau, 2002; Kurdek, 2008). These similarities between different-sex and same-sex relationships have led some close-relationships researchers to conclude that relationship science should not move forward as if its core principles would be qualitatively different in the lives of same-sex couples (Finkel et al., 2017). However, there are some important ways in which same-sex relationships are uniquely experienced that warrant specific attention.

The ways in which same-sex and different-sex couples structure and negotiate their relationships are often different. For example, same-sex couples tend to report more equitable distributions of financial, household, and childrearing responsibilities than different-sex couples (Diamond, 2006; Kurdek, 2005; Goldberg, 2013; Peplau \& Fingerhut, 2007; for an ethnographic study of family life in same-sex-headed households, see Carrington, 1999). As some researchers have posited (e.g., Adam, 2006; Degges-White \& Marszalek, 2007; Green \& Mitchell, 2002; Lannutti, 2008; Weston, 1991), due to the historically stigmatized status and lack of structural recognition for same-sex couples, same-sex relationships are often structured in ways that meet their practical needs through agreements that defy "tradition" (e.g., marriage) within heteronormative societies. Studies have shown, for example, that sexual minority individuals and same-sex couples have more open views surrounding monogamy and may be more likely to have open relationships than heterosexuals (Moors, Rubin, Matsick, Ziegler, \& Conley, 2014; van Eeden-Moorefield, Malloy, \& Benson, 2016). We review research on consensual nonmonogamy among same-sex couples later in this article.

Compared to understandings of how individuals structure their relationships and come to agreements surrounding issues like monogamy, very little attention has been paid to the psychological experiences of intimacy in samesex and different-sex relationships. Studies that have compared same-sex and different-sex couples on intimacyrelated constructs have primarily adopted behavioral indicators of intimacy (e.g., amount of time spent with a partner). The results of these studies indicate similar levels of intimacy across couples and sometimes higher levels among same-sex couples (Kurdek, 1998, 2004). At the subjective experiential and psychological levels, individuals in same-sex relationships seem to attribute the same level of meaning and importance to their pursuits of intimacy and do not demonstrate differences when compared to heterosexuals in regard to the prominence of intimacy in defining the meanings of their relationship experiences (Frost, 2011a, 2011b).

\section{Unique Experiences of Same-Sex Couples}

Despite the overwhelming amount of research that indicates same-sex relationships do not meaningfully differ from different-sex relationships in most important outcomes of relational health and functioning, differences do exist in two key aspects (Diamond, 2006): gender and stigma. The nature of interactions in same-sex and different-sex relationships is unique because partners in samesex couples share the same cisgender identity. Early findings in the American Couples Study (Blumstein \& Schwartz, 1983) indicated that female couples demonstrated the lowest frequency of sex relative to differentsex couples and male couples, who reported the highest levels. Several clinicians and researchers have offered hypotheses for this finding, referencing evidence from other research that women tend to demonstrate lower levels of libido, sexual frequency, and sexual assertiveness relative to men, but such findings have since been challenged (for a review, see Nichols, 2004). Nevertheless, this finding led to some theorizing of the potential existence of what was called "lesbian bed death," in which sexual desire and frequency of sexual activity were thought to decline in female couples rapidly over time due to unhealthy levels of merger, for which there has been a tremendous amount of clinical discussion but little empirical evidence to support (e.g., Frost \& Eliason, 2014). Also, the gender similarity in same-sex relationships has been cited for female and male couples demonstrating more equitable levels of distribution of housework and greater role equality in samesex relationships relative to different-sex relationships (for a review, see Diamond, 2006).

Important questions remain unanswered regarding the role of gender in shaping the unique experiences of samesex relationships. For example, female same-sex relationships between partners who identify as "butch" (i.e., masculine presenting) and "femme" (i.e., feminine presenting) may manifest important relational gender dynamics even though both partners may identify as cisgender women. In fact, Balsam and Szymanski (2005) found a small but significant association between femme identification and experiences of LGB-specific victimization in relationships. In the only population-based study of female relationships to address gender-role dynamics, Rothblum, Balsam, and Wickham (2018) found that gender-role identities tend to 
structure relationship pairings (with butch-femme and androgynous-androgynous pairings being most common), but gender-role pairings did not impact relationship quality. They found that femme-identified women reported more autonomy in their relationships, and that partners of femme-identified women reported doing less housework, further noting the complexities of gender dynamics in same-sex couples. However, beyond these two studies, relatively little research has examined the nuances of gender expression and gender roles in cisgender women's relationships despite the importance of gender role to gender identity in general (Eves, 2004; Levitt \& Horne, 2002). These gender dynamics within same-sex relationships are increasingly important to examine, given gender and sexual fluidity (e.g., Diamond, 2017). The changing nature of sexual identity labels - away from exclusive lesbian and gay monosexual identities, toward plurisexual queer and pansexual identities - has shifted the foundations of gender and sexuality in ways that may further differentiate female and male same-sex couples from different-sex couples (e.g., Galupo, Davis, Grynkiewicz, \& Mitchell, 2014).

Another way in which the intimate relationships of same-sex couples are unique concerns the consequences of the continued stigmatized status of same-sex relationships in most areas of the world. Given the global focus on same-sex marriage over the past two decades, much of the body of research on same-sex couples has focused on how stigma as a contextual factor shapes their experiences and well-being. Stigma exists at multiple levels, including structural stigma in the form of unequal opportunities for legal recognition (Hatzenbuehler, 2016), which stigmatizes all intimate relationships that fall outside of the heterosexual norm. Although many countries in Europe and North America provide equal access to legal relationship recognition, most countries in the world do not provide equal legal recognition to same-sex and different-sex couples, including some developed Western countries (e.g., the United Kingdom, Italy, Australia). This historically differential legal treatment is one way in which the experiences of same-sex couples are uniquely different from different-sex couples.

Like different-sex couples, not all same-sex couples desire to get married (Rothblum, 2005). In addition, some sexual minority individuals may experience a high degree of uncertainty regarding their own developmental trajectories and expectations in regard to relationship formation and milestone achievement (e.g., anniversaries) due to the historical lack of social recognition and value of intimacy in same-sex relationships (Kertzner, 2001). Indeed, there has been a considerable amount of debate about whether access to legal marriage will lead to positive outcomes for same-sex couples or whether it could exert negative pressures on same-sex couples to conform to heteronormative standards (Diamond, 2017). However, large proportions of sexual minority individuals have been found to report a desire for long-term romantic relationships and to marry their partners, should same-sex marriage be an available legal option (Baiocco, Argalia, \& Laghi, 2014; D’Augelli, Rendina, Sinclair, \& Grossman, 2007; Herek, 2006; Rostosky, Riggle, Rothblum, \& Balsam, 2016).

Regardless of the research evidence on intimacy and same-sex relationships, negative stereotypes regarding the meaning of intimacy in sexual minority individuals' lives and relationships create and reaffirm heterosexist opportunity structures, which privilege heterosexuals' opportunities to express and achieve intimacy while impeding the intimacy-related goals of sexual minority individuals (Frost \& LeBlanc, 2014; Herdt \& Kertzner, 2006; Meyer \& Dean, 1998). In addition, the lack of full public support for equal relationship recognition, which remains the case even in countries with legal equality, illustrates that society constructs same-sex relationships as lesser than different-sex relationships not just on a political or legal level but on a moral level as well (Frost \& Gola, 2015). Exposure to this social discourse of devaluation likely has an effect on people in same-sex relationships above and beyond their denial of equal legal recognition (Frost \& Fingerhut, 2016; Herdt \& Kertzner, 2006; Herek, 2006). The denial of equal rights associated with partnership establishes same-sex couples as second-class citizens in such contexts and likely diminishes social and psychological well-being for people in same-sex relationships (Hatzenbuehler, McLaughlin, Keyes, \& Hasin, 2010; Herdt \& Kertzner, 2006; King \& Bartlett, 2006).

Most countries continue to discriminate against samesex couples in providing access to legal recognition, and same-sex couples are often reminded of this fact by the news media, social media, and in conversations with their neighbors, family, and coworkers in daily interactions (Frost \& Fingerhut, 2016). This experience constitutes minority stress (i.e., stress specific to the experience of occupying a sexual minority status in the context of heterosexism; see Brooks, 1981; Frost et al., 2017; LeBlanc, Frost, \& Wight, 2015; Meyer, 2003; Russell \& Richards, 2003; Riggle, Thomas, \& Rostosky, 2005; for a broader historical discussion of minority stress, including ethnic minority stress, see Meyer, 1995) that likely has additional negative effects on well-being and relationship quality in same-sex relationships (Frost \& Fingerhut, 2016). Because these devaluing discourses call into question the general value of intimacy and relationships in sexual minority individuals' lives, their negative impact is likely to be felt by many people pursuing same-sex relationships, regardless of their desire to be legally married or publicly committed. Indeed, barriers to the achievement of intimacyrelated goals stemming from interpersonal (e.g., friends, family) and structural (e.g., laws, religious institutions) sources have been shown to partially explain health disparities between sexual minorities and heterosexuals (Frost \& LeBlanc, 2014).

The effects of negative political and societal discourses surrounding same-sex intimacy may further impact 
couples' experiences in relationships. Sexual minorities in same-sex relationships experience stigmatization specific to their relationships on an interpersonal level (Diamond, 2006; Frost \& Meyer, 2009; Green \& Mitchell, 2002; Peplau \& Fingerhut, 2007). People in same-sex relationships experience greater stress related to not being accepted and being misunderstood by other people in their lives, especially by their families, compared to single sexual minorities (Lewis, Derlega, Griffin, \& Krowinski, 2003). These interpersonal stressors, along with other forms of discrimination and victimization, are associated with decreased intimacy and relationship quality (see Doyle \& Molix, 2015; Rostosky \& Riggle, 2017b).

Coleman, Rosser, and Strapko (1992) pointed out that the anxiety, shame, and devaluation of sexual minority people and oneself as a sexual minority person are likely to be most overtly manifested in interpersonal relationships with other sexual minority individuals. Studies have empirically demonstrated that similar stigma-related processes interfere with same-sex relationships in numerous ways, such as decreasing relational functioning, increasing conflict among partners, and decreasing overall relationship satisfaction (for a review, see Rostosky \& Riggle, 2017b). Although these studies have established a general connection between stigma-related processes and relational functioning and well-being, none have yet fully investigated what factors may mediate the associations between stigma and relational functioning and well-being.

In truth, many same-sex couples are in happy, healthy, and rewarding relationships despite continuing stigma at the societal level (for a review, see Rostosky \& Riggle, 2017a). Some couples may see the stigma that society continues to attach to their relationships as a challenge rather than a threat, which ultimately benefits their relationship through complicated mechanisms of resistance and adaptation (Rostosky, Riggle, Gray, \& Hatton, 2007). Specifically, some couples utilize psychological meaningmaking strategies that frame experiences of prejudice and discrimination as something initially negative that, in fighting against and overcoming, reaffirms their bond to each other and brings them closer together as a couple (Frost, 2011b, 2014). Additional research shows that same-sex couples attribute many positive qualities of their relationships to the fact that they are in a same-sex relationship. For example, sexual minority individuals attribute the ability to enjoy egalitarian relationships and experience freedom from heteronormative gender roles as positive qualities potentially resulting from being in a same-sex relationship (Riggle, Whitman, Olson, Rostosky, \& Strong, 2008; Rostosky \& Riggle, 2017a).

\section{Queering the Study of Same-Sex Relationships}

Same-sex relationships defy conventional notions of normative relationships in their very definition. The normative standard that evolved over the course of human history across cultures has been the ideal of two individuals of different binary cisgender identities, namely male and female (Rubin, 2011c). Same-sex relationships challenge this regime of relational normativity, and as homosexuality gradually shifted from the frame of pathology to that of legitimate social identity (Hammack et al., 2013), the cultural and legal recognition of these queer relational forms assumed a central place in advocacy and empirical inquiry on relationship diversity. Much of the research on same-sex relationships has been guided by the political aim to destigmatize such forms and, in the process, redress the psychological injustice of structural and direct violence against sexual minorities (e.g., Frost \& Gola, 2015; Frost \& Ouellette, 2004; Herek, 2006) - the source of health and mental health disparities for sexual minorities (Meyer, 2003).

As we have suggested, there remains much to be done to legitimize same-sex relationships on a global scale. However, with legal recognition in an increasing number of nations and waning cultural disapproval of same-sex relationships and homosexuality more generally, new research questions emerge in the study of same-sex relationships that go beyond matters of comparison to different-sex relationships and the documentation of stigma. For example, we suggest that greater attention ought to be paid to the study of how contemporary same-sex couples engage with notions of relationship normativity and social mainstreaming now that, to some extent, their relational forms are not legally disadvantaged in the same ways they were in the past.

Although we consider same-sex relationships as "queer" within the proposed paradigm (because they do not conform to heteronormativity), same-sex couples may increasingly view themselves as fitting within normative notions of "traditional relationships" (e.g., monogamous and married), as legal recognition may increase the cultural legitimacy of such relational forms. Once relegated to "gay ghettos"- as much out of a need for security as a desire to reside within a minority community (Levine, 1979) many same-sex couples may feel that they can now live safely "assimilated" into predominantly heterosexual communities, though this sentiment likely varies by numerous intersecting factors such as race, ethnicity, class, gender identity and expression, and region (see Ghaziani, 2014).

Future research ought to examine the decision-making processes and experiences of same-sex couples as they engage with normativity. Why do some same-sex couples marry while others prefer alternative ways to recognize their union? Why do some reside within sexual minority communities while others do not? Why do some seek to have children, and in what myriad ways? How do experiences and social and psychological well-being differ for same-sex couples who make different decisions about such matters? What diverse forms do same-sex-headed families assume? How do same-sex couples' experiences vary at the intersections of other identities, like race, class, faith, and age? A queer paradigm upends the traditional 
focus of research on same-sex relationships on matters of equivalence with different-sex relationships toward interrogation of intimate diversity in all its forms. Such an approach replaces concepts of normativity, a relic of 20thcentury thinking in gender and sexuality, with assumptions of diversity, fluidity, and possibility.

Same-sex relationships framed as passionate or intimate friendships rather than romantic dyads also warrant renewed study. Diamond $(2000,2002)$ and Thompson (2007) have documented these types of same-sex relationships among sexual minority women, revealing the way in which they merge traditional friendship scripts with romantic scripts and some forms of physical intimacy. Such relationships can assume a role in young women's experience of sexual fluidity by providing a safe context for them to explore same-sex intimacy (Thompson, 2007). What other forms do such same-sex friendships take? To what extent do men of diverse sexual orientations also experience such same-sex intimacy? There is some evidence to suggest that, as homophobia declines and conceptions of masculinity expand to allow for same-sex intimacy, even men who identify as heterosexual experience passionate friendship with other men (e.g., Anderson, Adams, \& Rivers, 2012; Anderson \& McCormack, 2015).

These are just a sampling of new questions we believe ought to be addressed in 21st-century inquiry on same-sex relationships. Although there is greater diversity now in the extent to which same-sex relationships are legally recognized and culturally supported, stigma and subordinate status endure in much of the world, along with resulting health inequalities (e.g., Williams \& Mann, 2017). Documentation of the impact of this diversity on individuals and couples offers an important new line of inquiry for the study of relationships, which is needed to inform attempts to address these issues through policy, public health, educational, and other community- and individual-level interventions. The assumption of an explicitly queer approach to the study of same-sex relationships in the 21st century shifts our lens away from normativity toward recognition and interrogation of intimate diversity.

\section{Transgender and Gender-Nonbinary Intimacies}

The first axiom of a queer paradigm recognizes that intimacy may occur among individuals of any gender identity. In this section, we review research on relationships in which one or more individuals identify as transgender or as gender nonbinary, genderqueer, or a similar label that defies binary conceptions of gender. Although cisgender and transgender individuals may share a process of personality development in terms of self-categorization (Tate, Youssef, \& Bettergarcia, 2014), the endurance of cissexism (i.e., the privileging of cisgender identities and denigration of transgender or gender-nonconforming individuals; Serano, 2007) and transphobia suggest that the experiences of transgender and gender- nonbinary individuals in relationships might diverge from cisgender individuals in same-sex relationships.

As Pfeffer (2017) noted in her book on queer families, "Trans people, their partnerships, and families ... throw our social identity taxonomic classification systems into wonderfully perplexing disarray" (p. 133). Such queer relational forms are rooted in a lived experience of resistance to existing taxonomies of gender, sexuality, and identity through an affirmative process predicated on authenticity. They raise a host of new and compelling questions about the diversity of human relationships in the 21 st century.

It is important to note the extraordinary diversity of possible queer relational forms that may fall within the domain of trans intimacy. The term transgender encompasses a number of distinct identities and experiences, all of which are united in their fundamental critique of the notion that natal sex (or sex assigned at birth) is destiny with regard to gender identity, gender presentation, or the lived experience of gender. In spite of this commonality, transgender individuals represent diverse groups, and caution must be taken not to consider their experience in a uniform way (Dargie, Blair, Pukall, \& Coyle, 2014; Factor \& Rothblum, 2008).

We recognize that language within the umbrella category of transgender identities is evolving, and so our attempt here should not be considered exhaustive. Such identities include individuals historically called transsexual, or more recently trans binary, and typically referred to as trans men (assigned female sex at birth and now identifying as male) or trans women (assigned male sex at birth and now identifying as female). Individuals who identify as genderqueer, gender nonbinary, and/or gender fluid are all also often considered within the larger transgender umbrella (Teich, 2012). These individuals tend to reject the gender binary and do not identify as male or female (see Richards et al., 2016). They experience transphobic discrimination (Harrison, Grant, \& Herman, 2012) and report a strong sense of connection to the larger transgender community (Factor \& Rothblum, 2008), though their experiences in relationships are likely distinct from transgender binary individuals in ways that are beginning to be empirically examined (Galupo, Pulice-Farrow, Clements, \& Morris, in press; Pulice-Farrow, Bravo, \& Galupo, in press).

Transgender and other diverse forms of gender identity challenge normative notions of human relationships, which have historically been predicated on a binary notion of gender (Galupo, Henise, \& Mercer, 2016). They also create a seemingly endless number of configurations to consider the plurality of potential queer relational forms. For example, possible monoamorous configurations include a transgender man and a cisgender woman, a transgender woman and a cisgender man, a transgender man and a cisgender man, a transgender woman and a cisgender woman, a gender-nonbinary individual and a transgender man, a gender-fluid individual and a cisgender woman, and so on. 
When one adds the layer of sexual orientation and the history of a transgender person's sexual subjectivity, the nature of these queer relational forms becomes even more complex (e.g., Devor, 1993; Galupo et al., 2016, 2015). A trans man may have previously identified as a butch lesbian and may have been in a relationship with a femme lesbian prior to gender affirmation transition. As a woman, this individual might have identified as a lesbian but may now identify as heterosexual or queer. A trans woman may have previously identified as a heterosexual man, may have been masculine presenting, and may have been married to a heterosexual woman prior to gender affirmation transition. As a woman, this individual might now identify as a lesbian. The point is that the relational experiences of transgender individuals are characterized by a fluid and historical consideration of both gender and sexual identity. Personal and relational narratives may not assume a simple linear form for these individuals and their partners, and researchers must be especially sensitive to the diverse possibilities of lived experience for transgender individuals and their partners.

Research on queer relational forms in which one or more individuals identify as transgender or gender nonbinary is in its relative infancy but has increased substantially in recent years. A number of recent studies have examined the experience of friendship for transgender individuals, studying such issues as friendship experience across diverse gender identities and sexual orientations (Galupo et al., 2014), disclosure of transgender identity and status (Galupo, Krum, Hagen, Gonzalez, \& Bauerband, 2014), and experience of microaggressions (Galupo, Henise, \& Davis, 2014). Here, we limit our review to relationships that involve sexual and/or romantic intimacy. We highlight three primary areas of research that are dominant in the existing literature: (1) identity and sexual subjectivity, (2) minority stress experiences and processes, and (3) navigating normativity.

\section{Identity and Sexual Subjectivity}

One of the most significant and unique features of trans intimacies is that at least one partner in the relationship has undertaken a gender identity shift away from the sex assigned at birth. The identity development process of transgender individuals fundamentally involves a shift not only in gender identity and presentation but also in sexual and romantic subjectivity, which often results in a shift in sexual identity label (Galupo et al., 2016; Katz-Wise, Reisner, Hughto, \& Keo-Meier, 2016; Sanger, 2010). Because a dichotomized notion of gender underlies the primary way in which sexual and romantic attraction has been historically conceived (Galupo et al., 2016), a transformation in gender identity may reorient romantic and sexual subjectivity.

Trans individuals typically report significant stress associated with changes in sexual orientation or attraction as they transition to affirm gender (Mizock \& Hopwood, 2016). For example, the shift in being "read" as a lesbian to being read as a heterosexual man might be confusing and challenging, as it may involve misrecognition. Trans individuals often report discomfort with traditional sexual orientation labels because of the static and binary conception of gender underlying such labels, often preferring the label queer, which explicitly challenges all forms of normative identity categorization (Katz-Wise et al., 2016; Kuper, Nussbaum, \& Mustanski, 2012; Mizock \& Hopwood, 2016). Studies suggest that both transgender men and transgender women are more likely to report sexual attraction to women, though notable numbers report attraction to gay men (Devor, 1993; Factor \& Rothblum, 2008). One study suggests that transgender men may experience more sexual fluidity compared to transgender women (Katz-Wise et al., 2016).

Challenges to identity or self-understanding in trans intimacies have often been studied from the perspective of the cisgender partners of trans individuals. In a qualitative study of 16 cisgender females whose partners transitioned from male to female (i.e., they are now partnered to trans women), feelings of uncertainty and confusion in sexual orientation and maintenance of a heterosexual identity emerged (Alegría, 2013). Most of these cis women maintained a heterosexual identity and explained ongoing attraction to their partners as an exception (e.g., "situational" lesbianism). Some ( $n=3,19 \%)$ reported no longer engaging in sexual activity with their partners. Most $(81 \%)$ reported an "evolving" perspective on physical intimacy, actively attempting to maintain sexual relations. A more recent qualitative study with 21 partners (who identified as both cisgender and transgender) of transgender individuals found similar themes, including issues around physical and emotional intimacy, changing sexual orientation labels, safety concerns, feelings of isolation, and new appreciation of the gender spectrum (Platt \& Bolland, 2018).

Probably the most studied configuration of trans intimacy has been relationships between trans men who previously identified as butch lesbians and cis women who identify as femme lesbians. The construction and maintenance of gender identity is critical in these relationships, particularly as trans men undergo the process of gender affirmation transition. Ward (2010) highlighted the "gender labor" that femme women do in their relationships with trans men to "validate and celebrate their partners' masculinity and to suppress the complexity of their own gender and sexual subjectivity in the service of this goal" (p. 237). Trans men reported a desire for their femme partners to behave in ways that support their masculinity (e.g., being "the girl" in the relationship, "forgetting" their partner's past identity as female, and denying the status of their relationship as "transgender"; Ward, 2010). Femme partners in Ward's (2010) study reported confusion, resentment, and hard work in these relationships, with challenges to 
their own sense of gender and sexual identity. By contrast, Pfeffer $(2010,2017)$ found that cis partners of trans men engaged in disproportionate labor but justified it as an intentional act of personal agency consistent with thirdwave feminist ideology. It is important to note that some in these types of relational configurations may not view themselves or their relationships as necessarily "queer" (Platt \& Bolland, 2018). For example, trans men who identify as heterosexual and are partnered with a heterosexual-identified cisgender woman may view their relationship as "straight" or "heterosexual."

In her research with the cisgender women partners of trans men, Pfeffer $(2012,2014,2017)$ revealed the way in which trans intimacies necessarily involve challenges to identity and self-understanding. Most notable is the issue of whether cis partners continue to identify as lesbian once the notion of being in a same-sex relationship is no longer relevant or appropriate, given their partners' male gender identity. The notion that they might identify as straight or heterosexual is not appealing to such women, as their sexual and romantic orientation is generally directed toward masculine women. Pfeffer $(2012,2014,2017)$ found that these women increasingly (and especially among younger cohorts) prefer the label queer to signify their nonnormative sexual identities, as well as the term queer to refer to the nonnormative nature of their relationship. More research is needed to understand the distinctions between those relationships defined by partners as queer versus those intentionally defined in traditional binary terms such as straight, gay, or lesbian.

\section{Minority Stress Experiences and Processes}

Although transgender individuals have unique experiences related to identity, stress, and health compared with their cisgender LGB peers (Dargie et al., 2014), studies suggest that transgender individuals are just as impacted by minority stress as cisgender LGB people (e.g., Timmins, Rimes, \& Rahman, 2017). The minority stress framework can be applied not just to individuals but also to relationships; in other words, individuals of diverse sexual or gender identities experience structural violence, but relationships as social units do as well. Much of the limited research conducted on trans intimacies has revealed the experience of minority stress on account of the nonnormative nature of the relationship.

In a survey of couples consisting of trans women and their cisgender male partners, Gamarel, Reisner, Laurenceau, Nemoto, and Operario (2014) discovered high reports of relationship stigma. They found that higher reports of discrimination were associated with lower levels of relationship quality, suggesting a possible impact of explicit stigma on the relationship. They noted that minority stress impacts not only individuals but also dyads as relational units.
The nature of structural violence and explicit prejudice for trans individuals often represents a form of homophobic transphobia in which gender and sexuality are conflated (i.e., trans individuals are read as gay and harassed for their gender nonconformity). In their study of 45 individuals who identified as transgender or gender nonconforming, Mizock and Hopwood (2016) discovered the common experience of homophobic transphobia, as well as the experience of heteronormative gender-role pressure, which placed stress on the relationships of trans individuals.

The larger ideological context of cissexism, which denies the legitimacy of transgender identity and is embodied in such transphobic policies as "bathroom bills" (see Wernick, Kulick, \& Chin, 2017), creates a pervasive situation of explicit prejudice for trans individuals and their partners. The minority stress processes that are activated include internalized stress about body image and gender presentation; stress related to concealment, disclosure, and misrecognition; internalized stigma; stigma/rejection expectations; stigma or exclusion from the sexual minority community; and social support. We review the limited literature on each of these processes in turn.

As transgender individuals undergo a process of affirmation in which their gender presentation comes to align with their internal experience of gender identity, they experience both psychological benefits and challenges. Issues of body image, objectification, and minority stress intersect for trans individuals and their partners (Velez, Breslow, Brewster, Cox, \& Foster, 2016). Pfeffer (2008) revealed the way in which many trans men experience significant body dysphoria, and she proposed the concept of relational body image to highlight the way in which cisgender female partners of trans men may actually internalize negative sentiments about their own female bodies through the course of their relationships. Body modification, especially during the period of gender affirmative transition, can create considerable stress for trans individuals and their partners, as they must negotiate criticism and reaction from those who may not be supportive (Pfeffer, 2017). Gender presentation more broadly has been identified as a source of stress for trans individuals, who must constantly navigate issues of (mis) recognition and transphobic reactions (e.g., Levitt \& Ippolito, 2014). However, research also reveals the benefits of the experience of gender affirmation transition for transgender individuals, as their sense of gender identity becomes more consistent with their experiences of their own bodies (e.g., Prunas et al., 2017; Schimmel-Bristow et al., 2018). The ability to manifest one's gender with greater authenticity is associated with numerous social and psychological benefits for transgender people (e.g., Martinez, Sawyer, Thoroughgood, Ruggs, \& Smith, 2017).

In a larger cultural context of cissexism and transphobia, trans relationships involve heightened stress around concealment and disclosure. Members of a relationship are regularly in heightened states of information control around self-presentation (Goffman, 1959, 1963), as the disclosure of their status as a trans relationship could be not only 
discrediting but also dangerous. Nuttbrock et al. (2009) reported on a survey of two cohorts of 571 trans women in New York City, finding a higher likelihood of disclosure in their younger cohort (under age 40) and among "achieved" (e.g., friends or chosen family) rather than "ascribed" (e.g., family of origin) relationships. In their survey of more than 1,200 trans people in the United States, Iantaffi and Bockting (2011) found that trans men were more likely to have disclosed their trans identity to primary partners than were trans women.

While studies focus on experiences of concealment and disclosure among trans individuals, no studies of which we are aware have systematically examined concealment or disclosure stress at the dyadic or relational level. Studies have, however, examined issues of misrecognition among trans relationships. In her research with cisgender women partners of trans men, Pfeffer (2012, 2014, 2017) discovered the common phenomenon of these couples being misrecognized as straight or heteronormative because the gender presentation of the two partners conformed to binary femininity and masculinity. The cisgender women in these couples especially experienced this misrecognition as a source of stress because they did not identify with heterosexuality. Pfeffer (2017) described the way in which these couples intentionally identified as queer to avoid this misrecognition.

A particularly challenging minority stress process occurs when trans individuals or their relationship partners internalize the dominant discourse of cissexism and transphobia, which privileges cisgender identities and denigrates gender nonconformity. Studies reveal that internalized transphobia is associated with lower selfesteem (Austin \& Goodman, 2017; Iantaffi \& Bockting, 2011) and more rigid stereotypic gender beliefs (suggesting a more rigid internalization of the gender binary; Iantaffi \& Bockting, 2011). No studies to our knowledge have examined internalized stigma in the context of a relationship in which one or more partners identify as transgender. It seems likely, however, that internalized stigma would be associated with relationship dissatisfaction and possibly higher likelihood of relationship dissolution, as well as other negative relational outcomes.

Minority stress is characterized by a persistent vigilance about the possibility of stigma or rejection (Meyer, 2003). Like internalized stigma, stigma expectations have not been studied at the relational level but have been studied at the individual level. For example, research suggests that trans individuals experience fear of rejection on an ongoing basis, particularly with sexual or romantic partners (Iantaffi \& Bockting, 2011). It is important to note that transgender individuals experience stigma and exclusion not only from a cissexist and heterosexist society but also from the mainstream lesbian, gay, and bisexual community (e.g., Pfeffer, 2017; Weiss, 2003).
Within the minority stress framework, social support functions to moderate the potential impact of minority stress processes on negative health and mental health outcomes (e.g., Frost, Meyer, \& Schwartz, 2016; Meyer, 2003). The need for social support is especially strong for those trans individuals who affirm their gender through a transition process. In her study of trans people in the United Kingdom, Hines (2006) highlighted the importance of relationships in continuity and adjustment during the transition period. In their study of diverse trans people in the United States, Levitt and Ippolito (2014) found that open communication about sex and gender was key to the long-term success of their intimate relationships. Pfeffer (2017) highlighted the challenges that cis female partners of trans men have in finding a sense of community and support, as some are rejected from the lesbian community once their partners decide to transition.

\section{Navigating Normativity}

While trans intimacies offer a clear challenge to normative relational forms, there are ways in which members of trans relationships must uniquely navigate normativity. The focus of research in this area has been on gender performance and the extent to which trans relationships reproduce a traditional gender binary. For example, in her study of trans people and their partners in the United Kingdom, Sanger (2010) discovered a "normalization of binary gender categories" in which individuals seek to conform to normative ideals of masculinity and femininity in both self-presentation and behavior within the relationship. She found that older participants in her study were especially likely to appropriate or embrace heteronormative ideals of relationships.

To the extent that individuals in trans intimacies aspire to a binary gender identity, these relationships might assume what appears to be a normative form. As noted, cis women and trans men in relationships must navigate the complexity of a relationship once defined as explicitly queer (butch and femme lesbian), now negotiating the trans man's desire to embody masculinity and be identified as male with the cis woman's desire to maintain a lesbian or queer identity (e.g., Pfeffer, 2017; Ward, 2010). Pfeffer (2012) proposed the concepts of normative resistance and inventive pragmatism to describe the strategies cis women in these relationships use to navigate normativity. Normative resistance involves explicit identification as queer and often polyamorous to intentionally mark the relationship as nonnormative (e.g., Pfeffer, 2012). Inventive pragmatism refers to strategies intended to access legal marriage, parenthood, and reproductive technologies in the context of being misrecognized or technically categorized as a normative heterosexual couple.

Trans intimacies are diverse in their configurations, and while some, such as those studied by Pfeffer (2017) and Ward (2010), may be more likely to "pass" as 
heteronormative, others may be more likely to be immediately read as queer or transgressive with regard to gender presentation. Regardless, individuals in trans forms of intimacy must make decisions about the way in which they engage with normative notions of gender and sexuality. Research is needed to examine the diverse ways in which multiple configurations of trans intimacies respond to normativity.

It is important to note that research on individuals who identify as genderqueer, gender nonbinary, or gender fluid is especially limited. There is evidence that these individuals constitute a distinct population with regard to the experience of gender, sexuality, and mental health (see Richards et al., 2016). Recent research has begun to address the experience of individuals who identify as genderqueer, gender nonbinary, or gender fluid in intimate relationships, highlighting the importance of partners' validation, affirmation, and motivation to learn about the nonbinary experience (Galupo et al., in press; Pulice-Farrow et al., in press).

\section{Plurisexual Intimacies and Sexual Fluidity}

Normative assumptions about human intimacy presume that romantic and sexual desire occur in a singular manner (i.e., one is either attracted to the same or different sex) and remain static across the life course (Diamond, 2008; Rust, 2002). These assumptions are rooted in a binary, monosexual, essentialist conception of sexual orientation and sexual identity (DeLamater \& Hyde, 1998; Hammack, 2005). A queer paradigm rejects these assumptions, instead positing the possibility of plurality (i.e., attraction to multiple gender identities simultaneously) and fluidity (i.e., change in desire and/or identity) across the life course. The queer relational forms we review in this section are those that embody this possibility and are mainly undertaken by individuals who may be classified as plurisexual (i.e., attracted to multiple genders) rather than monosexual (i.e., attracted to one gender) and identify as bisexual, pansexual, and sexually fluid, and straight, "heteroflexible," and "mostly" straight, respectively (see Galupo, Ramirez, \& PuliceFarrow, 2017).

\section{Bisexual, Pansexual, and Sexually Fluid Intimacies}

Although people who identify as bisexual (i.e., attracted to both binary gender identities of male and female) or pansexual (i.e., attracted to multiple gender identities, including nonbinary identities) in their sexual identities (see Callis, 2014), desires, and behaviors are present in research on same-sex relationships, very few studies have investigated the potentially unique experiences of intimacy these individuals experience. This dearth of research is especially troublesome given that research on the psychology of sexual orientation generally tends to minimize, ignore, or even deny the existence of the experiences of bisexual and pansexual individuals (Flanders, 2017; Rust, 2002). Also, within sexual and gender minority communities, bisexual (and perhaps pansexual) individuals often report experiences of discrimination from other community members (Hayfield, Clarke, \& Halliwell, 2014) and lower levels of community connectedness potentially as a result (Kertzner, Meyer, Frost, \& Stirratt, 2009).

One explanation for this omission from research may be that intimacy in bisexual and pansexual individuals' lives is often defined from and compared to the point of view of homosexuality and/or heterosexuality rather than as the subject of study in its own right (Gustavson, 2009; Pennington, 2009). Thus, external frames are imposed upon their relational forms and lived experiences of intimacy, rather than building new understandings of the ways that intimacy is experienced as unique to those who identify as bisexual or pansexual. These frames have also arisen in bisexual individuals' own identities in relation to their partners. For example, bisexual women face stigmatization and must negotiate negative stereotypes from straight male and lesbian female partners (DeCapua, 2017; Hayfield et al., 2014). Also, among bisexual men, the "legitimacy" of being bisexual is more likely to be recognized by partners of younger men; older men tend to conform to more heteronormative ideals in relationships (Anderson, Scoats, $\&$ McCormack, 2015). Perhaps because bisexual and pansexual individuals have been stereotyped as "hypersexual" and having multiple partners (or not existing at all except in transition to a monosexual identity such as gay, lesbian, or straight), their relationships have not been treated as deserving of specific empirical attention (Gustavson, 2009; Rust, 2002).

The small amount of research that does exist has tended to focus on bisexual individuals' experiences relative to heterosexual and gay and lesbian individuals. These studies, often with serious methodological challenges associated with defining and sampling the bisexual population (e.g., response bias, lack of accurate sampling frames, and inappropriate comparison groups; Meyer \& Wilson, 2009; Rothblum, 2006), report relatively few differences between bisexual and heterosexual and gay and lesbian individuals on expectations and desire for "love preferences and ideals," as well as the desire to be in and the prevalence of relationships (Engel \& Saracino, 1986; Rust, 2002). However, some findings do indicate that bisexual individuals tend to be less concerned with jealousy and "fidelity" in their ideal close relationships than heterosexual and lesbian and gay individuals (Engel \& Saracino, 1986). Bisexual individuals tend to report lower rates of monogamy in their relationships, although studies tend to challenge popular stereotypes of bisexual individuals, noting that very few tend to be in simultaneous relationships with male- and female-identified individuals (for a review, see Rust, 2002). Further, some research on the experiences of bisexual-identified women in different-sex relationships indicates that bisexual women are able to attain satisfaction with male relationship partners while still expressing aspects of their 
bisexual identities via sexual relationships with other women (Reinhardt, 2011).

Additional research suggests that bisexual women who are in relationships with only a single male partner tend to be less open about being bisexual and more exposed to binegativity (i.e., stigma about bisexuality) compared to bisexual women who are in relationships with male and female partners or only a female partner (Molina et al., 2015). These experiences of binegativity have been in turn associated with poorer health in the form of depression and substance use (Molina et al., 2015). Taken together, these findings highlight the potential constricting and deleterious effect that the imposition of a heteronormative frame can have for bisexual women: Not being able to fully express bisexual identity and behaviorally engage with male and female partners appears to be detrimental to bisexual women's health. More research is needed to determine whether similar effects exist for bisexual men.

It should be noted that some individuals who are categorized as bisexual in the research literature often identify as bisexual in combination with other sexual identity labels (e.g., queer) or may consider themselves to be pansexual (Callis, 2014; Elizabeth, 2013). Pansexual and other plurisexual identities are increasingly being used among younger generations as sexual identity labels (Galupo et al., 2014, 2017), with some studies estimating a prevalence of the term among adolescents and emerging adults of around 3\% (Gamarel, Walker, Rivera, \& Golub, 2014; McInroy \& Craig, 2012). However, research has yet to focus specifically on the potentially unique experiences of pansexual and other plurisexual individuals in intimate relationships. This is important, given that queer-identified women report more expansive and varied sexual behavior and desire patterns and are more likely to experience fluidity in their sexual identities than bisexual-identified women (Mereish, KatzWise, \& Woulfe, 2017). Thus, future research concerned with intimacy among individuals who have nonbinary sexual identities should attempt to oversample pansexual individuals and treat their experiences of intimacy as worthy of study in their own right rather than combining bisexual and pansexual individuals based on assumptions of similarity (Flanders, 2017).

As more insight is gained into the phenomenon of sexual fluidity (Diamond, 2008; Katz-Wise, 2015; KatzWise \& Hyde, 2015), new questions emerge regarding the ways in which shifting sexual identities, desires, and behavior patterns orient individuals and their partners within the context of intimate relationships (e.g., Better, 2014; Mereish et al., 2017). Indeed, research has shown that sexual identities can shift in the context of or resulting from intimate relationship formation and dissolution. For example, Diamond's (2008) longitudinal study of sexual fluidity in a cohort of sexual minority women found that $67 \%$ experienced changes in the target of their sexual attraction over a 10-year period. Further cross-sectional survey research by Katz-Wise (2015) replicated this finding ( $62 \%$ of women reported sexual fluidity in attraction) and extended it to men $(52 \%$ reported sexual fluidity in attraction). Diamond's (2008) longitudinal research documented instances in which women changed their sexual identities in response to falling in love with a particular individual. Such a phenomenon may in part reflect the experience of being "demisexual" (i.e., individuals who do not experience sexual attraction until they experience an emotional bond with someone else; Galupo et al., 2014) and further calls attention to unanswered questions surrounding the intimate relationship experiences of individuals who are not monosexual in their identities, behaviors, and desires.

The heightened visibility of individuals who identify as bisexual or pansexual or who report sexual fluidity has challenged categorical notions of sexual orientation as both fixed (in the case of sexual fluidity) and focused on the gender of a partner (in the case of bisexuality and pansexuality). Yet research on relationships has not sufficiently interrogated the unique experiences of individuals with these diverse identities and forms of desire. Because bisexual, pansexual, and other plurisexual identities challenge the expectation that sexual and gender identities neatly align with relationship configurations (e.g., heterosexual individuals are in different-sex relationships and sexual minorities are in same-sex relationships), a queer paradigm is needed to dismantle this narrow and limiting assumption underlying much of the previous research in relationship science (Gonzalez, Ramirez, \& Galupo, 2017). We call on social scientists to engage with diverse relational forms, both to represent and amplify these unique experiences and also to provide us with a more complete picture of the diversity of human intimacy.

\section{Queer Heterosexuality}

At the core of notions of relational normativity is the ideal of different-sex attraction, so much so that critiques of same-sex attraction have long positioned it as in conflict with the "natural" design of human bodies (Corvino, 2013). Reflective of a growing recognition of the complexity and diversity of human intimacy, even individuals whose attractions are oriented toward the normative ideal of a different sex are currently defying received notions of heterosexuality. Several scholars have argued that the success of social and political movements for sexual and gender diversity, accompanied by declining "homohysteria" (Anderson, 2011; McCormack \& Anderson, 2014) and heightened visibility of sexual minorities in an increasingly "post-closeted" culture (Dean, 2014), has resulted in new opportunities and a new level of intentionality in the construction and expression of relations among heterosexuals (e.g., Morgan, 2012; Silva, 2018).

A larger context of declining homophobia (McCormack, 2012; McCormack \& Anderson, 2014) and support for 
sexual and gender identity diversity (Keleher \& Smith, 2012) has likely created new possibilities for intimate expression among individuals who identify as heterosexual. There is evidence that substantial numbers of heterosexuals question their sexual orientation and undergo a process of exploration prior to committing to a heterosexual identity. For example, in samples of college students in the United States, Morgan and colleagues found that $53 \%$ of men and $67 \%$ of women who identified as exclusively straight reported questioning their sexual orientation (Morgan, Steiner, \& Thompson, 2010; Morgan \& Thompson, 2011). There is also evidence that a more inclusive version of masculinity has emerged in the United States and the United Kingdom in which physical intimacy between men, including same-sex behavior, is no longer necessarily considered in conflict with masculinity or an indicator of gay identity (Anderson, 2008, 2011; Anderson et al., 2012; Anderson \& McCormack, 2015; Bridges, 2014; Dean, 2014; McCormack \& Anderson, 2014).

We highlight two notable indicators of this "queering" of normative heterosexuality that have been recently documented by researchers: the emergence of individuals who identify as mostly or primarily straight (sometimes termed heteroflexible) and the proliferation of straight-identified men who have sex with men. We recognize that the notion of "queer heterosexuality" may be considered contentious, as an investment in heterosexual identity over an explicit plurisexual identity such as bisexual or pansexual would seem to signal an affinity with normativity. Nonetheless, we contend that a commitment to heterosexual identification may better speak to the lived experience of individuals with strong different-sex desire, and that their willingness to challenge the binary notion of sexual orientation through their intimate practices signals a unique critique of normativity. As Silva (2018) noted, "Normativity can be challenged not just from the fringes of sexuality and gender (e.g., LGBTQ+ individuals) but also unintentionally from within the institution of heterosexuality itself" (p. 85).

Multiple studies have confirmed the unique profile of individuals who identify as mostly or primarily straight. For example, Thompson and Morgan (2008) found significant differences between college women who identified as mostly straight versus exclusively straight, bisexual, or lesbian on such dimensions as sexual attraction, sexual behavior, and sexual identity development. In their survey of almost 1,800 individuals recruited online through social media, Vrangalova and Savin-Williams (2012) found support for a five-category classification of sexual identity (heterosexual, mostly heterosexual, bisexual, mostly gay/ lesbian, and gay/lesbian), with men more likely to identify with the exclusive ends of the continuum.

In a major review of research on individuals who identify as mostly straight, Savin-Williams and Vrangalova (2013) argued that there is sufficient evidence to consider this group a distinct sexual identity category because they show a unique profile of sexual and romantic interests and characteristics. These individuals report a small degree of same-sex attraction and only occasional same-sex behavior. They constitute a substantial prevalence in the population and report a mostly straight identity as subjectively meaningful. Consistent with the historical argument that movements for sexual and gender diversity have created space for the complication of heterosexuality (e.g., Dean, 2014), Savin-Williams and Vrangalova (2013) found evidence of both age and cohort effects, whereby younger people were more likely to identify as mostly straight. Savin-Williams (2018) has recently discovered evidence that an additional category, called primarily straight, also exists and indexes meaningful differences with individuals who identify as exclusively or mostly straight.

Individuals who identify as mostly straight are to be distinguished from those who identify as straight but engage in sex with members of the same sex (Carrillo \& Hoffman, 2018). Most of the research in this area has been conducted with straight-identified men (e.g., Carrillo \& Hoffman, 2018; Silva, 2017, 2018; Ward, 2015), as research on same-sex relations among straight-identified women has typically been situated in the literature on bisexuality and sexual fluidity (reviewed previously; see Diamond, 2008). Because same-sex behavior among straight-identified men is less frequently associated with changes in labeling or identification compared with women (cf. Silva, 2018), we suggest that this queer relational form is better situated within a flexible or evolving understanding of heterosexuality (Carrillo \& Hoffman, 2018).

Kinsey, Pomeroy, and Martin (1948) shocked the U.S. public with their finding that $37 \%$ of men reported some same-sex activity in adulthood. There is considerable evidence that, similar to the era in which Kinsey et al. (1948) conducted their research, a substantial number of heterosexual-identified men engage in some form of same-sex activity. For example, Anderson's (2008) study of former high school football players found that $40 \%$ acknowledged same-sex sexual activity in college. We distinguish between the rise in nonsexual, "homosocial" intimacy among straight-identified men that has been increasingly documented in an age of declining homohysteria (Anderson et al., 2012; Anderson \& McCormack, 2015) and explicit sexual behavior, although we acknowledge that both sexual and nonsexual intimacy among men represent queer relational practices in relation to compulsive or hegemonic masculinity (see Connell \& Messerschmidt, 2005; Pascoe, 2005; cf. Ward, 2008).

In the early 2000s, the phenomenon of same-sex behavior among straight-identified men was documented within a racialized, pathologizing discourse of the "down-low" in which it was claimed that Black and Latino men were largely rejecting sexual minority labels and placing their female partners at risk for human immunodeficiency virus (HIV) and other sexually transmitted infections (STIs) (e.g., DenizetLewis, 2003; King, 2004). Two key assumptions about this 
phenomenon have now been discredited. First, there is insufficient evidence to suggest that straight-identified men who seek sex with other men are "actually gay." In fact, these men appear to be heavily invested in heterosexuality as an identity and an institution (e.g., Silva, 2018; Ward, 2008, 2015). Second, there is now substantial evidence that straight White men also seek sex with other men in significant numbers, so the phenomenon is by no means unique to men of a particular race or ethnicity (e.g., Carrillo \& Hoffman, 2016, 2018; Ward, 2015). Even the discourse of the down-low has been appropriated by straight White men seeking sex with other men (Robinson \& Vidal-Ortiz, 2013).

Rather than viewing heterosexual-identifying men who seek and have sex with other men as closeted gay men, it appears more appropriate to view them in a manner consistent with their own self-identification: primarily attracted to women (Carrillo \& Hoffman, 2016, 2018) and heavily invested in heterosexual culture and its institutions and rituals, such as marriage and family (e.g., Persson et al., 2017; Reback \& Larkins, 2010). Many of these men are also heavily invested in versions of masculinity that emphasize sex between men as appropriate and as actually bolstering masculinity in some contexts (e.g., Silva, 2017, 2018; Ward, 2008, 2015). In fact, Ward (2008) has argued that these men ought not to be viewed as "queer" precisely because of their explicit investment in a heteropatriarchal form of normativity that denigrates women.

The motivations of straight men who seek sex with other men are diverse, and their experience of intimacy with other men also assumes a range of intensity. While one study found economic necessity (i.e., "gay for pay") as a motivation (Reback \& Larkins, 2010), others have found that straight men seek sex with other men for reasons such as stress relief (Carrillo \& Hoffman, 2018), the desire to engage in more aggressive sex thought to be undesirable to women (Carrillo \& Hoffman, 2018; Ward, 2015), or casual sexual release perceived to have no romantic attachment and thus less "threatening" than sex would be with female partners (Reback \& Larkins, 2010; Reynolds, 2015; Silva, 2017, 2018; Ward, 2008, 2015). Many of these men also report a desire for sex with other men as a form of "male bonding," revealing the way in which these men view same-sex behavior as consistent with a form of heterosexual masculinity (Reynolds, 2015; Silva, 2017, 2018; Ward, 2008, 2015). These men explicitly reject a version of mainstream gay male culture and its institutions that they see as inconsistent with masculinity (e.g., Silva, 2018; Ward, 2008, 2015). It is precisely for this reason that Ward (2008) challenged the notion that this form of heterosexuality ought even to be considered "queer," for its investment in normative ideologies of masculinity and heterosexuality seems central. We recognize the legitimacy of this contention, yet we view these intimate practices as still "queer" in the sense of challenging a cardinal principle of heteronormative masculinity: that sexual behavior must be reserved for women.
The consideration of potentially queer forms of heterosexuality is relatively new and continues to evolve as social scientists document the ways in which individuals who identify with heterosexuality as a relational form defy its hegemonic construction. Central to the production of compulsory heterosexuality has been a patriarchal ideology and accompanying cultural institutions that have historically subordinated women (Rich, 1980). In this sense, Ward's (2008) critique of male "heteroflexibility" in some forms appears consistent with compulsory heterosexuality. Yet the erosion of the "one-time rule" of homosexuality that once constrained same-sex intimacy among heterosexual men seems to have opened up space for the complication of male heterosexuality in ways that are potentially liberating, especially if decoupled from patriarchal versions of masculinity (e.g., Anderson, 2011; McCormack \& Anderson, 2014). Further research is needed to interrogate the diverse ways in which straight-identified individuals are engaging with normative and nonnormative discourses and practices related to gender, sexuality, and relationality. The number of works recently published in this area suggests this vital work is under way.

\section{Polyamory and Consensual Nonmonogamies}

In contrast to the normative assumption that restricts intimacy to two individuals (i.e., monoamory, dyadic monogamy), a queer paradigm posits that intimacy may occur with multiple partners simultaneously with consent. In this section, we review research on three forms of consensual nonmonogamy: (1) swinging, (2) open relationships, and (3) polyamory. We maintain this division to reflect the distinction between vocabularies used by particular communities at particular historical moments. For example, "swinger" communities emerged in the 1960 s and 1970s among heterosexual couples seeking to "swap" partners for a brief period, often a single night (e.g., Denfeld \& Gordon, 1970). By contrast, "poly" communities emerged in the 1990s, principally among heterosexual and bisexual communities (e.g., Barker, 2005), to reflect an interest in more enduring connections with multiple partners (e.g., Anapol, 1997). The discourse of "open relationships" has been most common in the gay male community (e.g., Bonello \& Cross, 2010). Our choice to maintain a division within our review reflects these distinctions, but we recognize that all forms of consensual nonmonogamy encounter potential stigma due to the pervasiveness of mononormativity - the widely held conviction that monogamy represents the most "natural," moral, and beneficial form of human intimacy (Barker \& Landridge, 2010; Conley, Moors, Matsick, \& Ziegler, 2013).

Similar to notions of compulsory heterosexuality (Rich, 1980), monogamy has been framed as a prescriptive cultural ideal to the exclusion of other forms of intimacy (Emens, 2004; Ritchie \& Barker, 2006). Individuals appear 
to internalize a hierarchy of value that positions monogamous relationships as more favorable or superior to various forms of nonmonogamy (Conley et al., 2013; Grunt-Mejer \& Campbell, 2016). Even many who practice consensual nonmonogamy appear to privilege monogamy as an ideal, likely internalizing stigma about their own nonnormative practices (Conley et al., 2013). Over time, forms of consensual nonmonogamy have grown from "deviant" practices (e.g., Denfeld \& Gordon, 1970) into full-fledged identities and communities (e.g., Barker, 2005). Our review considers each of the three common forms of consensual nonmonogamy in the context of their historical emergence in the literature.

\section{Swinging}

Swinging emerged as a documented form of consensual nonmonogamy among heterosexual couples in the 1970s, as the cultural revolution of the time challenged received notions of sexual exclusivity (Hunt, 1974; Smith \& Smith, 1970). Denfeld and Gordon (1970) argued that the institutionalization of swinging emerged over the 20th century with shifting views on female sexuality (e.g., greater recognition of women's sexual desire), premarital and marital sex (e.g., greater recognition that sex could be for recreation and not just procreation), and technological developments related to contraception (e.g., the pill).

Swinging, or "comarital sex" (Jenks, 1998; Smith \& Smith, 1970), is a particular form of consensual nonmonogamy in which married, heterosexual couples agree to swap partners (Buunk \& van Driel, 1989). The limited research on swinging has focused on the characteristics of individuals and couples who engage in this practice or who explicitly identify as swingers. Swingers tends to be White, politically conservative (except with regard to sexuality), not religious, highly educated, middle or upper class, and in professional or white-collar careers (e.g., Jenks, 1985; for a review, see Jenks, 1998). Jenks (1998) proposed a social psychological process model to explain the development of interest in swinging, emphasizing strong sexual desire, significant premarital sexual exploration, liberal sexual predisposition, a low degree of jealousy, and contact with a larger swinging community. Research suggests that marital satisfaction and other indicators of relationship quality are similar for swingers and nonswingers (see Rubel \& Bogaert, 2015).

Although swingers, like other practitioners of consensual nonmonogamy, may be less likely to experience jealousy (Jenks, 1998), the management of emotions among swinging couples likely requires careful intention and communication. In one of the few explicit studies on swinging since Jenks's (1998) review, de Visser and McDonald (2007) studied the management of jealousy among four active heterosexual swinging couples in England. Through in-depth couple and individual interviews, they discovered specific strategies couples used to manage their emotions.
They emphasized the importance of a strong dyadic identity (i.e., emotional commitment to each other and to the couple as a unit) with explicit communication about feelings in the context of negotiated rules regarding both sexual and emotional connection with extradyadic partners.

Kimberly and Hans (2017) examined the process married couples experience as they transition from monogamy to swinging. They interviewed 32 individuals from 16 couples and analyzed their data using a grounded theory approach, which resulted in a conceptual model of the path from initial interest to fulfillment of a swinger lifestyle. They found that the maintenance of marital satisfaction involved a commitment to long-term friendship, shared activities, enhanced trust, and open communication. Their analysis also revealed the importance of rules to construct and maintain boundaries of consensual sexual activity outside the marriage.

Although swinging continues to exist as a specific form of consensual nonmonogamy (see Bergstrand \& Sinski, 2010; Kimberly \& Hans, 2017), it is less well documented than more recent forms, such as open relationships and polyamory. We suspect that use of these newer vocabularies and forms of consensual nonmonogamy are driven by historical change in the meanings of intimacy, sex, and marriage. Swinging emerged as a practice and an identifiable community at a particular historical moment. While it retains some usage, other forms have become more common in the popular lexicon of relationships.

\section{Open Relationships}

While the concept of swinging and a swinger identity and community seems to have developed in the context of heterosexual marriage, the idea of open relationships has flourished largely in sexual minority communities, especially among gay men. Open relationships are typically characterized by exclusivity of emotional commitment to one primary partner but sexual nonexclusivity (e.g., Bonello \& Cross, 2010). Such relationships are distinguished from polyamory in their tendency to make a firm distinction between sexual and emotional commitment and to generally prohibit or discourage multiple emotional or romantic attachments. Almost all research using the vocabulary of "open relationships" has occurred among gay men, with studies of nonmonogamous different-sex or same-sex female couples using the language of either swinging or polyamory (e.g., Munson \& Stelbaum, 1999; Sheff, 2005, 2006; cf. Bettinger, 2005).

Open relationships have long been a part of the gay male community and have been well documented since the mid20th century (Adam, 2004; Bell \& Weinberg, 1978; Blumstein \& Schwartz, 1983; Sadownick, 1996). Having decreased somewhat with the acquired immunodeficiency syndrome (AIDS) epidemic when sexual exclusivity emerged as a common HIV prevention mechanism (e.g., Berger, 1990; Davidson, 1991; cf. Hickson et al., 1992), 
open relationships now thrive among gay men and appear to constitute a common form of relational structure (e.g., Haupert, Gesselman, Moors, Fisher, \& Garcia, 2017). Shifts in the meaning of HIV and the availability of new highly effective tools for prevention have likely created a sexual culture that facilitates open relationships among gay men (see Hammack, Frost, Meyer, \& Pletta, 2018).

Research comparing gay men in open versus monogamous relationships has consistently discovered no differences in relationship quality or satisfaction between types of relationship (Blasband \& Peplau, 1985; Bonello, 2009; Bricker \& Horne, 2007; Kurdek \& Schmitt, 1985; LaSala, 2004a). Gay men commonly report a desire for sexual variety and independence as motivating nonmonogamy in their relationships (e.g., Blasband \& Peplau, 1985; Bonello, 2009; LaSala, 2004b), and many gay men are either explicitly critical of monogamy or endorse the view that monogamy represents an unsustainable ideal (e.g., Coelho, 2011; Worth, Reid, \& McMillan, 2002). Relationships between gay men also change over time, with partners often beginning with monogamy but the expectation that the relationship will ultimately be open, given cultural norms and expectations within the gay male community (Philpot et al., 2018).

Research has examined strategies gay men in open relationships use to manage their emotions and the overall health of their relationships. Bonello and Cross (2010) identified three common strategies: compartmentalization, boundary affirmation, and secretiveness. Compartmentalization entails cognitive separation of the type of intimacy experienced with one's primary partner and that of other, purely sexual encounters (see also LaSala, 2004b). That is, the couple engages in emotional exclusivity with regard to intimacy, and sexual partners outside of the primary relationship do not involve romantic or emotional cognition. Boundary affirmation occurs when couples engage in casual sex together and, through this act, affirm the boundaries of their openness. Secretiveness entails a "don't ask, don't tell" strategy in which sexual activity outside the primary relationship is not disclosed between partners.

Although early research suggested that sexual exclusivity represents a continuum among gay male couples (Blasband \& Peplau, 1985), only recently have studies begun to move away from a simple dichotomy of "open" versus "closed." Hoff and Beougher (2010) interviewed 39 gay male couples in San Francisco about sexual agreements and relationship dynamics, discovering that these agreements were best understood on a continuum rather than in terms of discrete categories. Couples reported agreements that ranged from explicit monogamy (i.e., no sexual or emotional intimacy outside of the relationship) to varying levels of openness. For example, some couples described agreements that allowed for threesomes under certain conditions, while others allowed for sex outside of the primary relationship without the knowledge of the partner. Couples were largely motivated to have an agreement to provide structure or build trust within the relationship. Couples were not generally motivated to establish agreements for sexual health or HIV prevention reasons, which may be related to the shifting cultural meaning of HIV/AIDS among gay men (Hammack et al., 2018).

In a larger study of HIV risk and relationship agreements among 566 gay male couples in San Francisco, Hoff and colleagues found about equal numbers of monogamous $(45 \%)$ versus nonmonogamous (47\%) couples, and the majority of couples (64\%) reported explicit discussions regarding relationship agreements (Hoff, Beougher, Chakravarty, Darbes, \& Neilands, 2010). Only seroconcordant HIV-negative couples (who comprised 55\% of the sample) indicated HIV or STI prevention as a motivating factor in their agreements. While monogamous couples reported greater intimacy, trust, commitment, and attachment to their partners (as well as equality in the relationship), there were no differences between monogamous and nonmonogamous couples in relationship satisfaction or autonomy. Similar studies of gay men have not detected differences between relationship types on indicators of relationship quality (e.g., sexual satisfaction, communication, or frequency; Parsons, Starks, Gamarel, \& Grov, 2012) and health and well-being (Parsons, Starks, DuBois, Grov, \& Golub, 2013). Taken together, research on gay men in relationships suggests that consensual nonmonogamy is common, takes on many forms that are explicitly negotiated, and is associated with positive indicators of relationship quality.

An important future direction for research on open relationships is to expand to include other relationship configurations beyond gay men. Our review of the published literature revealed that studies of consensual nonmonogamy among other types of relationships tend to classify them within either swinging or polyamory. We suggest that the vocabulary of open relationships is probably more common among a diversity of couples than researchers may realize, and thus we believe future research ought to expand beyond its traditional focus on same-sex male couples.

\section{Polyamory}

Polyamory emerged as a concept in the 1990s with the growth of an intentional community committed to a form of nonmonogamy in which multiple emotional and sexual attachments are supported and valued. Prior to systematic empirical research, which commenced in earnest in the 2000s, several popular books were published that outlined a set of principles to anchor the polyamorous community (Anapol, 1997; Easton \& Liszt, 1997). These works outlined guidelines for ethical nonmonogamy, including consent, honesty, commitment, boundary setting, and agreement negotiation (Anapol, 1997; Easton \& Liszt, 1997).

Around the same time that empirical research on polyamory began to blossom, Emens (2004) presented a legal analysis emphasizing the question of why the marriage 
equality movement for same-sex couples should not include recognition of "plural marriages." She asked why the "numerosity requirement" of marriage (i.e., one man and one woman) was not a subject of challenge while the gender component was. She argued that resistance to polyamory is rooted in historical visions of Mormon polygyny, which conflicts with contemporary ideals and ethics of polyamory. Emens (2004) proposed five principles of polyamory based on analysis of the limited available data on the community: self-knowledge, radical honesty, consent, selfpossession, and privileging of love and sex over jealousy. She highlighted the way in which compulsory monogamy is enshrined in law.

Research on polyamory has examined the principles and practices of its practitioners (e.g., Klesse, 2006), the ways in which polyamorous individuals engage with normative ideas about relationships (e.g., Aviram, 2007), gender roles and dynamics in poly communities (e.g., Sheff, 2006), issues of legal recognition (e.g., Sheff, 2011), and issues of identity, community, and meaning (e.g., Barker, 2005). In one of the earliest empirical studies of polyamory, McLean (2004) interviewed 60 bisexual men and women in Australia, about $75 \%$ of whom were in consensually nonmonogamous relationships. Honesty and communication emerged as critical "ground rules" for relationship agreements. In a pattern that persists across most existing research on polyamory, McLean (2004) found the common configuration of a primary male-female dyad, with male partners preferring their female partners engage in samesex rather than different-sex relations (see also Sheff, 2006, 2011). Klesse (2006) interviewed 44 practitioners of polyamory in the United Kingdom, including gay and bisexual men and bisexual women, finding "love" and "friendship" as central to definitions of polyamory practitioners use to distinguish between practices of casual sex that occur in swinging. Klesse's (2006) participants emphasized the idea of polyamory as a form of "responsible nonmonogamy" to distinguish themselves from swingers.

An emphasis on the ethical behavior of polyamorists relative to others who practice nonmonogamy is consistent across other qualitative studies. Barker and Ritchie (2007) analyzed focus group data from women in nonmonogamous relationships in the United Kingdom, finding that participants likened polyamory to a feminist practice in which monogamy is explicitly critiqued for its historic oppression of women. Their participants challenged the common notion that polyamory primarily benefits heterosexual men. Wosick-Correa (2010) surveyed 343 practitioners of polyamory in the United States, finding that $96 \%$ have explicit agreements or rules within the relationship. She found that commitment emerged as a central concept in polyamorous relationships and that rule violations are not generally interpreted as "cheating" but rather as opportunities to renegotiate agreements. Wosick-Correa (2010) introduced the concept of agentic fidelity to describe the polyamorous form of commitment that "relies upon acute self-knowledge and choice exercised through the ability to express needs and boundaries" (p. 44). Conley and Moors (2014) highlighted four ways in which polyamory might benefit marital relationships: increasing social capital through connection beyond the dyad, better distributing household labor, enhancing communication, and safely managing attraction beyond the dyad. They suggested that marital quality could be enhanced through strategies in use by polyamorists for communication, household resource management, and conflict management (Conley \& Moors, 2014).

Research suggests that practitioners of polyamory are highly intentional as they engage with normative ideas about relationships. In their study of more than 60 women in monogamous, open, and polyamorous relationships, Pallotta-Chiarolli and Lubowitz (2003) revealed the way in which the relationships of poly women can often "pass" as normatively heterosexual, using the concept of "outside belonging" to describe the "border existence" of poly women. In their discourse analysis of online discussions, Web sites, and self-help books in the U.K. poly community, Ritchie and Barker (2006) discovered the way in which existing language that privileges mononormativity constrains potentials for polyamory. They highlighted the way in which the poly community has developed an alternative language that challenges or repudiates mononormative concepts of partnership, infidelity, and jealousy. For example, the reclaiming of the term slut among the poly community illustrates the way in which normative concepts are challenged (Ritchie \& Barker, 2006; see also Hardy \& Easton, 2017).

Polyamorists must also make decisions about marriage, which is a cultural and legal institution grounded in mononormativity or compulsory monogamy (Emens, 2004). Aviram (2007) interviewed 35 polyamory activists regarding marriage equality, finding them critical and distrustful of the law as a tool to recognize their relationships. Sheff's (2011) ethnographic research in poly communities in the United States identified the way in which practitioners of polyamory constructed alternative family forms (e.g., "chosen families"), commonly anchored by a male-female primary dyad and their constellations. She found that poly families typically had made some legal arrangements to protect themselves, but they were highly critical of marriage as an institution and, in contrast to same-sex couples, did not generally advocate for legal recognition through marriage. Similarly, Aguilar's (2013) ethnography of two communal groups of poly practitioners revealed the way in which group members engaged in "ideological work" associated with feminism and the repudiation of traditional institutions like marriage.

The study of gender roles and dynamics has figured prominently in research on polyamory, particularly because most poly communities studied have been composed chiefly of heterosexual men and heterosexual and bisexual women. Sheff's $(2005,2006)$ ethnographic research 
especially focused on gender dynamics. Sheff (2005) found that participation in a polyamorous community and explicit rejection of monogamy facilitated women's opportunities to question prescribed sex roles and gender norms more generally. Some women in her research did, however, report feelings of objectification by heterosexual men in the polyamory community who sought sex with multiple women. Yet many of her participants also noted strong emotional connections between female partners and the characterization of men in the community as "sex toys." Most women in Sheff's (2005) research reported a strong sense of power in the community, though some reported a more "traditional" power structure in which men relied upon women in the community to do the "emotion work" needed to develop and maintain relationships. Sheff (2006) noted the way in which the men in her study resisted hegemonic forms of masculinity. She introduced the concept of polyhegemonic masculinity as an idealized form of masculinity in the (straight) poly community in which men develop emotional intimacy and multiple relationships with women who also have other lovers. Hence, this form of masculinity is characterized by more emotional availability and lack of possessiveness and dominance compared to hegemonic heterosexual masculinity. Many poly men appear to espouse and practice more egalitarian, sex-positive, and gender-neutral relational styles (Sheff, 2006).

One of the most compelling issues studied among practitioners of polyamory focuses on issues of identity, community, and meaning. Barker (2005) interviewed 30 members of the bisexual polyamory community in the United Kingdom, discovering that participant discourse diverged with regard to the meaning of polyamory. While some of their interviewees considered polyamory to constitute an identity or sexual/relational orientation, invoking ideas of bioessentialism, others framed polyamory as an intentional practice, eschewing the notion of a "poly" sexual orientation or identity.

Based on interviews with 40 bisexual women in Toronto, Robinson (2013) argued that polyamory is better conceived as a "strategic identity" rather than a relational or sexual "orientation" that would imply immutability (see also Benson, 2017). Manley et al. (2015) compared 55 polyamorous individuals with 61 monoamorous individuals regarding sexual identity, attraction, and partnering behaviors at two time points seven months apart. They found that polyamorous individuals were more likely to identify their sexuality using nontraditional labels and nonpolar ways (e.g., bisexual and mostly straight rather than straight, lesbian, or gay) and that polyamorous women shifted their attractions at a higher rate than polyamorous men or monoamorous men and women. They argued that their findings suggest the need to consider relational identity as a concept and to further study diverse relationship identities.

The conception of polyamory as an identity or orientation versus a practice likely emerges from the legal debate on same-sex marriage in which advocates tended to advance a bioessentialist view of sexual orientation to argue for rights and recognition (Emens, 2004; Kitzinger \& Wilkinson, 2004). Existing research suggests that polyamorous communities are concerned with legal recognition but are often critical of marriage (e.g., Aviram, 2007; Sheff, 2011). Klesse (2014) reviewed this issue and argued that "the equation of polyamory with sexual orientation may undermine the disruptive potential of the category polyamory, achieve only selective protection under the law, obstruct the ability of poly movements to pursue broader alliances, and foster a politics of recognition at the expense of a more transformative political agenda" (p. 92). Similar to critiques of the marriage equality movement within the gay and lesbian community (e.g., Warner, 1999), Klesse (2014) suggested that integration into the existing institution of marriage might dilute the radical potential of polyamory.

Several notable critiques are warranted of the existing research on polyamory, chiefly on issues of sampling and representation. In spite of calls for greater representation of non-White samples (e.g., Haritawon, Lin, \& Klesse, 2006), the overwhelming majority of studies have examined largely White middle- and upper-class communities in anglophone (especially the United Kingdom and the United States) societies (Sheff \& Hammers, 2011). There may be deterrents to participate in research among individuals of nondominant race or social class based on fears of discrimination, tokenism, or the rejection of poly communities as largely White (Sheff \& Hammers, 2011).

In addition, most research has focused on communities consisting chiefly of heterosexual men and bisexual or heterosexual women. Polyamorous communities consisting chiefly of gay men have been perhaps understudied or typically classified within the open relationships literature, as noted (see Bettinger, 2005). Polyamory within the lesbian community has also been relatively understudied (see Munson \& Stelbaum, 1999).

As Easton and Hardy (2009) noted, the practice of polyamory likely varies across different communities of sexual minority groups. Polyamory among other gender and sexual minority groups, such as those who identify as asexual, transgender, gender nonbinary, and other diverse sexual and gender minority identities, has not been systematically studied (see Richards, 2010). An intersectional approach (Crenshaw, 1989) that takes race, class, gender, sexual identity, ability status, and other social identities into account is much needed within the literature on polyamory.

Dyadic monogamy represents a privileged and hegemonic relational form enshrined in law (Emens, 2004). Polyamory is both morally derided and without legal recognition as a legitimate form of intimacy (Emens, 2004). Polyamorists thus occupy a place of structural disadvantage and social stigma that might impact their psychological well-being. Research on polyamory would benefit from the application of a minority stress lens that examines the 
way in which polyamorists navigate mononormativity and a social structure that explicitly denigrates polyamory. We know little about how individuals who identify as polyamorous manage prejudice, stigma, and stress related to concealment and disclosure.

\section{Kink/Fetish/BDSM Intimacies}

The fourth axiom of a queer paradigm is that intimacy may be characterized by consensual asymmetry based on power exchange either during particular intimate acts or on a full-time basis. While same-sex relationships and forms of consensual nonmonogamy challenge normative ideas about gender and numerosity in intimate relations, respectively, kink/fetish relationships challenge normative ideas about power, equality, and symmetry. Visibility of kink practices and relationships has increased dramatically in the 21 st century, in part as the result of popular media successes such as Fifty Shades of Grey (James, 2012). Though impossible to fully estimate in terms of prevalence, in a nationally representative sample of more than 19,000 Australians conducted in 2001-2002, 1.8\% of respondents reported involvement in BDSM (Richters, de Visser, Rissel, Grulich, \& Smith, 2008).

Here we use the terms kink, fetish, and BDSM relatively interchangeably, as we recognize the diverse usage to convey largely synonymous meanings. Ortmann and Sprott (2013) noted that the terms kink and kinky emerged from within the community rather than representing language that was imposed from the medical or scientific community, which is the case with both fetish and sadomasochism. In practice, all of these labels are in general use within diverse communities of kink practitioners.

Our review of the literature focuses on the defining features of kink relationships and the social and psychological experience of individuals in such relationships. We note that overt discussion of kink relationships is extraordinarily rare in the literature. Rather, most research emphasizes kinky "practices" or "scenes" in a way that implicitly delegitimizes the connection among partners as ephemeral or linked mainly to individual pleasure. We call for increased research on relationships that are explicitly defined as kinky.

Our review highlights four common features or experiences of kink relationships. First, such relationships are typically characterized by recreational power exchange. Second, they are consensual and collaboratively scripted. Third, they are typically embedded in a larger community of shared meaning and practices. Finally, because such relationships may be stigmatized, individuals may experience minority stress related to issues of concealment, disclosure, stigma, and internalized kinkphobia.

\section{Recreational Power Exchange}

Central to most kink relationships and dynamics is the experience of power asymmetry and exchange, in contrast to contemporary normative relationships in which symmetry and equality are idealized though often not achieved (van Hooff, 2013). While early pathologizing perspectives assumed that the administration and receipt of physical pain was central to BDSM (e.g., Krafft-Ebing, 1886), research has consistently revealed that the core of kink dynamics is a desire for power exchange and role-play associated with power (e.g., Cross \& Matheson, 2006; Faccio, Casini, \& Cipolletta, 2014; for review, see Weinberg, 1987, 1994, 2006). Weinberg (1987) referred to the "ritualization of dominance and submission" as the "essence" of BDSM.

In one of the first nonpathologizing empirical studies of the kink community, Kamel (1980) conducted participant observation in the gay male leather community. He discussed scenes among men as role performances in which power differentials were enacted. Physical restraints, for example, were utilized to establish roles, and consensual humiliation was a central part of role performance. He argued that "leathermen" constitute a distinct subculture with ritualized norms around contact, action, and relationships, and he highlighted the development of a leatherman identity among gay men centering on masculine gender roles, progressing from disenchantment with the mainstream gay community. More recent research conducted by Mosher, Levitt, and Manley (2006) among gay leathermen found very similar patterns decades later, revealing the endurance of this subcultural identity.

Based on eight years of fieldwork in both straight and gay kink communities in San Francisco and New York, Weinberg, Williams, and Moser (1984) identified rolebased power exchange, consent, and mutual definition of roles as core features of kink dynamics. More recent studies have affirmed the centrality of power exchange as a core feature of kink relationships (e.g., Chaline, 2010). Taylor and Ussher (2001) interviewed 24 selfidentified sadomasochists in the United Kingdom and the Netherlands. Thematic discourse analysis of interview data revealed consensuality, unequal balance of power, sexual arousal, and compatibility of definition (i.e., mutual or collaboratively scripted roles) as definitional components of kink relationships and dynamics. Langdridge and Butt (2005) conducted a discourse analysis of BDSM community Web sites and forums, finding a primary emphasis on erotic power exchange. In their qualitative interview study of six self-identified leathermen, Mosher et al. (2006) found an emphasis on power exchange in relationships, but participant narratives highlighted displays of hypermasculinity and nurturance, as well as interdependence between partners regardless of dominant and submissive roles. Their findings support the notion that power exchange in kink relationships is collaboratively scripted and interdependent. Faccio et al. 
(2014) interviewed 50 heterosexual BDSM practitioners about the meaning of their sexual experiences, finding that participants described their activities as consensual "games" with specific rules. Their participants emphasized sexual pleasure and power exchange, often in relation to traditional gender roles between men and women (either affirming or reversing such roles).

Often the power exchange in kink relationships can take the form of explicit play with social categories of race, gender, or age (see Ortmann \& Sprott, 2013). Among self-identified dyke/trans BDSM practitioners, Bauer (2008) discovered a comfort with age, gender, and sexuality role-play over race or class play. Participants cited their strong commitments to anti-racism and other critical perspectives. Participants noted that this type of consensual power play represented a tool for personal growth and healing, providing a level of agency that contrasted to their everyday lives. In a more recent study of self-identified les-bi-trans-queer BDSM individuals in the United States and Europe, Bauer (2018) found that all who engaged in age or intergenerational play disavowed pedophilia and sexual abuse. Participants reported age play as an opportunity for exploration of difference in role.

The power exchange in kink relationships is best described as recreational in nature, as it involves the assumption of roles and often alternative personas, either strictly within sex or in a $24 / 7$ manner in the relationship context. In the only study that, to our knowledge, has examined the $24 / 7$ fetish relationship in depth, Dancer, Kleinplatz, and Moser (2006) surveyed 146 self-identified "slaves" about their relationships. Respondents reported high levels of relationship satisfaction and fulfillment and described specific rituals and behaviors enacted to maintain a slave mind-set on an ongoing, 24/7 basis. They reported the ability to step "out of role" when appropriate (e.g., in workplace settings). Their relationships were structured to safeguard their well-being, and they reported the ability to leave the relationship if they so desired.

Other studies have taken an explicit framing of kink relationships as a form of "play" or "serious leisure." Weiss (2006) conducted ethnographic fieldwork in San Francisco's heterosexual/pansexual BDSM community from 2001-2003. Based on her research, she argued that BDSM represents "working at play" and a "serious game" in which performance involving real-world structures of dominance (e.g., relating to gender) are enacted. Newmahr (2010) conducted a four-year ethnography of a BDSM community in the northeastern United States. Based on her research, she argued for a framing of BDSM practices as "serious leisure" rather than simply "kinky sex." She detailed the learning processes involved in play (e.g., learning the specifics of assuming a top/dominant role versus a bottom/submissive one), the self-actualization and self-expression involved, as well as empowerment and efficacy. She detailed the way in which many practitioners discuss their relations as involving a regeneration or renewal of self, overcoming trauma, and facilitating "flow" or optimal psychological experience (Newmahr, 2010).

In their recent study of "pup play" (i.e., simulating the role of puppies in sexual and social interaction) among gay and bisexual men in the United Kingdom, Wignall and McCormack (2017) argued that this newly popular form of kink activity and subculture can best be interpreted as "serious leisure" in Newmahr's (2010) framework. Through interviews with men who engage in pup play, Wignall and McCormack (2017) revealed the way in which this practice has transformed into a larger subcultural community with specific relational structures (e.g., "Handler/pup"). Their data revealed that this activity is not associated with zoophilia or bestiality in any way among practitioners but rather involves a type of role-play in which a "headspace" is achieved in both sexual and nonsexual activities. Journalistic accounts of this growing subculture also reveal a more elaborate social organization that would benefit from future study (e.g., specific role relationships among pups, such as "Alpha/beta"; the formation of family units organized as "Packs"; see Bannon, 2016; Bieschke, 2015).

\section{Consent and Collaborative Scripting}

The second defining feature of kink relationships documented in the literature is consent and collaborative scripting of roles. Central to all kink relationships and dynamics - and distinguishing them from expressions of power in abusive relationships - is the full consent of all participants (see Langdridge \& Barker, 2007; Weinberg, 1987, 1994, 2006). Individuals assuming a dominant or submissive role do so through explicit consent, and this feature of kink dynamics has long been documented in empirical studies (e.g., Weinberg et al., 1984). Studies of community Web site content (Langdridge \& Butt, 2004) and interview data from both straight (Faccio et al., 2014; Taylor \& Ussher, 2001) and gay (Chaline, 2010) kink practitioners consistently reveal the centrality of consent to understandings of BDSM relationships.

Key to the consensual nature of kink relations is the collaborative scripting of roles. Dynamics such as "Daddy Dom/little girl," "Master/slave," and "Handler/pup" (to name just a few of current popular relational forms) represent jointly constructed stories that involve role-play outside the boundaries of everyday life. The relationships are highly interdependent, and the power exchange of the relationship is typically play focused, as the dynamic requires the individual in the submissive role to consent for the exchange to occur. Research reveals that BDSM practitioners are fully aware of this interdependence (e.g., Mosher et al., 2006; Taylor \& Ussher, 2001; Weinberg et al., 1984).

Although some of these asymmetrical scripts rely on existing or historical power differentials related to race or 
gender and thus might appear problematic to those concerned with inequality (e.g., Linden, 1982), individuals in such relationships often report therapeutic value in coping with anxieties or traumas (e.g., Lindemann, 2011) and the opportunity to engage with and psychologically manage the experience of historical forms of dominance in safe and potentially therapeutic ways (see Bauer, 2008, 2018). Rather than power being forced on the relationship by implicit outside forces, the power exchange in kink relationships involves a performance that highlights the way in which all roles are fundamentally performative - an experience that participants in Mosher et al.'s (2006) study linked to the way in which the performative nature of gender is apparent in drag. These role performances often also represent reversals of historical dynamics of domination, as is the case in the relationship between a Mistress and a slave (Ortmann \& Sprott, 2013; see Faccio et al., 2014). These relationships can often involve an explicit grappling with and reframing of injustice as a form of pleasure and intimacy, giving participants a sense of power and agency over social forces like racism and sexism that are normally so systemic and widespread as to be beyond control (e.g., Lindemann, 2010). Centrally, these relationships are collaboratively scripted and consensual in nature, in contrast to popular representations such as Fifty Shades of Grey (James, 2012; see Barker, 2013). In fact, key aspects of kink relationships such as consent, direct communication, and benefits of power play stand to inform nonkink relationships in ways that may enhance satisfaction, pleasure, and safety (see Ortmann \& Sprott, 2013).

\section{Community and Cultural Meaning}

The third defining feature of kink relationships we highlight here is their grounding in a larger community that provides resources, support, and a system of cultural meaning. Early research on BDSM recognized the way in which kink practices were embedded in rich, alternative cultural systems existing apart from mainstream society. Much of this work has centered on the study of the gay male leather subculture (e.g., Brodsky, 1993; Kamel, 1980; Mosher et al., 2006; Rubin, 2011a; for a historical discussion of this kink community, see Ridinger, 2002). Kamel's (1980) ethnographic study of the gay leather community revealed the elaborate way in which subcultural norms were used to establish contact, action, roles, and specific sexual interests. Rubin's (2011a) ethnography of the Catacombs, a San Francisco sex club, emphasized the way in which the venue facilitated new forms of intimate connection among gay men, as well as the eventual formation of a mixed-gender BDSM community. Brodsky's (1993) retrospective ethnography of the Mineshaft, a gay male BDSM club in New York, emphasized the social and cultural function at the communal level for gay men at the time (1970s-1980s) who had encountered both the liberating era of the post-Stonewall "gay ghettos" (Levine, 1979) of the 1970s and the devastation of the AIDS epidemic in the 1980s (see Hammack et al., 2018). Brodsky (1993) argued that the kink practices of the Mineshaft were not strictly concerned with sexual gratification; they also represented transformative experiences through ritual participation in the creation of a gay male masculine culture and identity. A study of gay leathermen by Mosher et al. (2006) affirmed this central role of the kink community and its ritualized practices for the creation of a positive and meaningful identity. The men in their study identified leather communities as sites of interpersonal validation in which they achieved enhanced selfconfidence through unconditional acceptance, with the community's emphasis on family, fraternity, and brotherhood. Their interviewees viewed the gay leather community as more inclusive than the mainstream gay scene, with its emphasis on social status. In the leather community, men reported feeling united in their commitment to the kink subculture, with its alternative vision of gay male identity and sexuality (Mosher et al., 2006).

Studies have also revealed the significance of a kink community among heterosexual, bisexual, and pansexual individuals. Weinberg and Falk's (1980) early study of the social organization of BDSM for heterosexuals revealed the significance and value of community, chiefly for locating potential relationship partners. Like Kamel (1980) and Rubin (2011a), Weinberg and Falk (1980) identified the community as a site for cultural meaning, and they also noted that community organizations provided invaluable psychological resources for kinky individuals to understand their desires through a nonpathologizing lens. Hence, the community not only represents a cultural system and mechanism to locate relationship partners but also assumes a vital role in stigma management. More recent research affirms the significance of community for heterosexual and pansexual kinky individuals. For example, Weiss's (2006) ethnography revealed the emphasis on a collective community among practitioners in her study, and Barker (2013) has argued for an evolving understanding of consent among BDSM practitioners toward community, rather than solely individual or dyadic, responsibility.

As Weinberg (2006) noted and as our review here reveals, there is no singular "kink community," and the plural subcultures that exist are largely organized according to gender and sexual orientation. The limited research that has been conducted has revealed the meaning and value of community for diverse practitioners of kink. Further research is needed to document the diverse subcultures that have developed to support individuals in kink relationships.

\section{Stigma and Minority Stress}

The final feature of kink relationships we highlight centers on the potential experience of stigma and minority stress. Due to the way in which kink challenges normative ideas and structures about relationships, individuals who identify as kinky or are involved in kink relationships 
appear to experience many of the same minority stressors as sexual and gender identity minorities (e.g., Wright, 2006; see Ortmann \& Sprott, 2013). Research in this area, however, has been very limited and warrants further study.

The stigmatization of kink practices and relationships creates a context of structural disadvantage for kinky individuals in which their relationships are regarded as culturally inferior and their consensual activities considered illegal in some contexts (see Langdridge, 2006; Ridinger, 2006). In addition, kink relationships are stigmatized from several constituencies, including many communities typically seen as supportive of other forms of sexual diversity (e.g., gay and feminist communities). In these communities, objection to kink often comes either in the form of concerns about how the larger group is viewed from normative society (e.g., opposition to the gay leather scene from the mainstream gay male community; Kamel, 1980; Mosher et al., 2006) or about how the power exchange in kink relations defies values of equality (e.g., sex-negative feminist opposition to BDSM; see Rubin, 2011b; Wright, 2006). This societal stigma may result in prejudice and activate minority stress processes such as stigma expectations, stress related to disclosure/concealment, and internalized stigma (e.g., Lin, 2017; Stiles \& Clark, 2011). Consistent with minority stress theory (Meyer, 2003), these processes may in turn lead to elevated rates of depression and other negative mental health outcomes, although the limited research on mental health among kink practitioners has not indicated elevated rates of depression or other forms of psychological distress (e.g., Breslow, Evans, \& Langley, 1986; Moser \& Levitt, 1987; Richters et al., 2008). To our knowledge, no research has systematically examined a minority stress model among individuals who identify as kinky or who are engaged in kink relationships. Such research would be invaluable for our growing understanding of the social and psychological experience of kink. How do partners in kink relationships navigate kinkphobic prejudice and minority stress processes such as concealment/disclosure stress and internalized stigma? What distinctions exist in the experience or expression of kink/BDSM intimacies across diverse gender, race, class, and sexual identities?

\section{Asexual and Aromantic Intimacies}

One of the most enduring assumptions about intimate relationships is that they are inherently sexual and romantic. The growth of the asexual community and its heightened visibility has challenged this normative assumption, and the number of people who identify as asexual or on the "ace spectrum" calls for empirical attention to the unique experiences and issues of these individuals. The fifth axiom of a queer paradigm for the study of relationship diversity is that intimacy may occur in the absence or limited experience of both sexual or romantic desire.
We note that the language to describe diverse experiences and expressions of asexuality is in a state of emergence, but here we define some key terms (for further discussion of terminology, see Bogaert, 2012; Cowan \& LeBlanc, 2018). Asexual, or colloquially "ace," refers to individuals who do not experience sexual attraction to other individuals, though they are physiologically capable of sexual arousal and may engage in practices such as masturbation (Bogaert, 2012). Aromantic refers to individuals who do not experience romantic attraction to other individuals. Terms such as graysexual or demisexual (or grayromantic, demiromantic) refer to individuals located somewhere on what is colloquially called the "ace spectrum." These individuals report some degree of sexual attraction within a broader identification with asexuality (i.e., graysexual) or report being unable to experience sexual attraction unless romantically attracted to someone (i.e., demisexual). This review of terminology is not exhaustive, and we note that a common practice within the larger asexual community is to make a distinction between sexual and romantic desire.

A study of so-called "Boston marriages" (Rothblum \& Brehony, 1993) is often cited as the beginning of academic inquiry into the relationship experiences of asexual people. However, the study conceptualized nonsexual relationships essentially as lesbian marriages rather than identifying asexuality as its own category. This conceptualization is part of a tradition we described earlier of depicting lesbian relationships as less sexual than other relationship configurations, controversially termed "lesbian bed death" (see Blumstein \& Schwartz, 1983). Contemporary research on asexuality has abandoned this lesbian-only framework and has understood asexuality not as a single, stable category but instead as a spectrum that is often experienced fluidly and differently for people who are in relationships compared with those who are not (Scott \& Dawson, 2015).

Asexual-identified people often differentiate between sexual and romantic attraction to identify the forms that intimate relationships can take (Bogaert, 2006; Carrigan, 2011; MacNeela \& Murphy, 2015; Scherrer, 2008). Importantly, sexual and romantic attraction do not always "match" in terms of gender identity (Diamond, 2003), with some asexuals experiencing no sexual attraction toward anyone while experiencing romantic attraction toward men, or others reporting sexual attraction toward men but romantic attraction only toward women. Among asexual people, a wide variety of configurations of intimate relationships has been identified, from relationships with sexual partners which do involve consensual sex, even if sex is not explicitly desired (Brotto, Knudson, Inskip, Rhodes, \& Erskine, 2010; MacNeela \& Murphy, 2015), to romantic relationships without sexual activity and close relationships that resemble friendships (Scherrer, 2008). Some who identify as asexual may be responsive to sexual stimuli but choose not to seek intimate relationships at all (Bogaert, 2017). This distinction between sex and romance in relationships is also useful for understanding the relationships 
of nonasexual, nonaromantic people, who happen to be in a relationship that is primarily sexual or primarily romantic, such as "friends with benefits" relationships or companionate love (e.g., Garcia, Reiber, Massey, \& Merriwether, 2012; Sternberg, 1986).

Two of the most frequent questions asked by recent researchers in this area focus on whether there are gender differences in the prevalence of asexuality, and if people who self-identify as on the asexual spectrum are more or less likely to be involved in intimate relationships than sexual people. Findings on these questions have been mixed. Several studies have concluded that asexual-identified people are less likely to report being currently involved in an intimate relationship than sexual people (Bogaert, 2004; Yule, Brotto, \& Gorzalka, 2014), but at least one study has not been able to replicate this finding (Prause \& Graham, 2007). Likewise, van Houdenhove, Gijs, T'Sjoen, and Enzlin (2015) found that asexual men were more likely to be single than asexual women, while Prause and Graham (2007) and MacNeela and Murphy (2015) did not find gender differences in reported relationship status. Further research is needed to examine gender differences, especially beyond the binary of male-female.

While asexual people often experience stigma because of their relationships being devalued (Chasin, 2015) or for being ostracized or misunderstood (Robbins, Low, \& Query, 2016), recent research has identified a handful of relationship strategies used by people on the asexual spectrum to manage stigma. For example, some asexual people have found that BDSM-oriented relationships have provided them with tools to negotiate and demarcate the boundaries in their relationships, as well as to reject and change dominant scripts of sexual behavior (Sloan, 2015). By organizing these relationships around consensual BDSM, some asexuals have found the ability to experience intense forms of nonsexual intimacy. Others have found that engaging in the community of people who also identify on the asexual spectrum has provided them with the language to "make sense" of their relationships and desires (Chasin, 2015). Finally, other research has cited coming out to partners as a strategy to manage sexual conflicts, although the dissolution of those relationships has sometimes been a consequence of coming out (Robbins et al., 2016).

The emergence of a community of people who identify as asexual has taken the experience of little or no desire for sex as a core part of intimacy from the realm of pathology to a legitimate form of sexual diversity. With heightened visibility and community cohesion has come significantly more social science research on the meaning and experience of asexuality, yet we note that very little research has directly examined the experiences of asexual people in relationships. The unique relationships of asexual individuals constitute a queer relational form in need of legitimization through empirical documentation and systematic inquiry. What diverse forms of asexual and aromantic intimacies exist, and how do individuals make meaning of these relationships in a cultural context that privileges sex and romance? How do partners in asexual or aromantic relationships navigate stigma from multiple sources, including sexual and gender identity minority communities? What distinctions exist in the experience or expression of asexuality or aromanticism across diverse gender, race, class, and sexual identities?

\section{Chosen Families}

I am Pepper Lebeija, the legendary mother of the House of Lebeija... . It's important to me to be the mother because there's so many little kids that I have to look out for, although they don't listen to me, and they buck my authority. I still think I rule it pretty well.

In the opening minutes of Jennie Livingston's 1991 documentary Paris Is Burning, we are introduced to Pepper Lebeija, "the legendary mother of the House of Lebeija," and with that the concept of the drag house-a relational form intended to provide meaning, support, and mentorship in the context of stigma and rejection. Such configurations defy the normative assumption that the family represents a unit in which individuals are primarily linked by biological relation in a traditional nuclear structure. The sixth axiom of a queer paradigm is that the family unit as a social institution may be defined by intentionality, identity, and community connection rather than biological relation. We refer to such units as chosen families.

A hegemonic notion of the heteronormative family, defined in biological terms and composed of two different-sex individuals and their genetic offspring, has long defined the historical imagination (Coontz, 2016). Historical evidence suggests that this notion of family has always been grounded in a degree of mythology, hegemonic in its compulsory nature and in the way in which it supported patriarchy (Lindsey, 1981). Since at least the early 1980 s, social scientists have critically interrogated this construction and revealed the diversity of family forms that exist (e.g., Macklin, 1980). Lindsey's (1981) early analysis identified specific forms of chosen kin, including honorary kin (e.g., individuals unrelated by biology who assume labels such as "uncle" and "aunt"), workplace families, and friend networks as families.

In the 1980s and 1990s, the clearest empirical examples of chosen families appeared within gay, lesbian, and queer communities across the United States, including the community depicted in Paris Is Burning. Weston (1991) developed a historical argument related to gay and lesbian culture to explain the emergence of explicit chosen family forms in this era. The success of the gay and lesbian movement led to the formation of visible, organized urban communities in the 1970 s and to the ability of scores to come 
out at younger ages. Weston (1991) suggested that the formation of smaller units of social organization within gay and lesbian communities was likely in part a reaction to both the diversity and size of a visible sexual minority subculture. In addition, the AIDS crisis of the 1980s and 1990 s and the lesbian "baby boom" (i.e., the rise in lesbians choosing to parent) called for creativity in the provision of emotional support. It is also noteworthy that cultural stigma toward homosexuality and sexual diversity was extremely high across the United States at this time (e.g., Herek \& Glunt, 1988). Finally, this historical era was defined by universal prohibition of same-sex marriage, which required sexual minorities to envision alternative family forms to the heteronormative family. In short, the 1980s and 1990s were a period in which the chosen family form thrived in sexual minority communities out of social and psychological necessity.

This period has remained among the best documented for the study of chosen families. Weston's (1991) ethnographic study of lesbians and gay men in the San Francisco Bay area discovered that sexual minorities use a kinship discourse ("families we choose") in response to rejection from their families of origin. She argued that gay and lesbian kinship ideologies challenge conventional notions of kinship as fundamentally driven by biology. Key to motivations of her participants was the desire to signal a clear separation from the dominant heterosexual culture and to find support among other sexual minorities.

Studies conducted with gay men and lesbians in the 1990s discovered similar patterns of chosen family configuration, especially organized around friendship networks. Nardi's (1999) study of gay men highlighted the use of the language of family to describe friend networks and the reliance of these men on friends for social and emotional support. In her study of midlife lesbians in the United States (ages 40 to 65, born between 1935 and 1961), Weinstock (2000) discovered a strong ethic of "friends as family." She argued that this cohort (whose ages in 2018 range from the late fifties to early eighties) was unique in experiencing early development in prefeminist and pre-LGBT rights eras, young adulthood during the rise of feminism and gay liberation, and at the time of her study experiencing midlife with the consequences of these movements. In their study of nonheterosexuals in the United Kingdom conducted in the mid-1990s, Weeks, Heaphy, and Donovan (2001) discovered the use of family terminology to describe friendship circles. They defined families of choice as "creative adaptations to rapid change based on voluntary association" (p. 20), situating their analysis in the historical context of late modernity and its changes in the meaning of intimacy (e.g., Giddens, 1992; Plummer, 1995).

As the AIDS crisis shifted in meaning, with treatment and prevention advances and marriage equality moved to center stage in the gay and lesbian political movement by the turn of the 21 st century, research on chosen families as alternative kin structures largely receded. However, a small body of research on the topic has examined such factors as the composition and diverse configurations of chosen families, the social psychological processes associated with chosen family formation, and the role of chosen families in the provision of social and emotional support.

Most research on the composition and configuration of chosen families has continued the research line of the 1990s (e.g., Nardi, 1999) by emphasizing friend networks. Stacey's (2005) ethnographic study of gay male family forms in Los Angeles from 1999 to 2003 discovered highly diverse configurations of family and kinship. She argued that gay men are unique in their intentionality and creativity in constructing alternative family structures: "Gay male genres of intimacy and kinship represent more visible and experimental responses ... to the irreversible postmodern family condition that beckons and bedevils us all" (pp. 1931-1932).

Much research on the significance of friends as family has continued to focus on older cohorts of sexual minorities who developed in eras of especially heightened stigma and inequality, especially in terms of the ability to choose more normative family forms through marriage. De Vries and Megathlin (2009) interviewed 53 gay/lesbian and 106 heterosexual men and women 50 to 88 years old about the meaning of friendship and family, finding that gay men and lesbians were more likely than heterosexuals to include cognitive (trust, loyalty) and affective (care, compatibility) processes in their definitions. More recently, Traies (2015) reported on a mixed-methods study of more than 400 lesbians aged 60 to 90 in the United Kingdom, finding a strong emphasis on friendship and social networks, including relationships with ex-partners, in the provision of support for emotional and mental health problems.

The social support function of friend networks or explicitly identified chosen families has been especially well documented in recent studies. Research on friendship among transgender individuals suggests the significance of LGBT friends as a benefit for creating a sense of shared community and family belonging (Galupo et al., 2014). In an online survey of 698 adults in Canada and the United States of diverse sexual orientations, Blair and Pukall (2015) examined differences in past experiences of relationship disapproval from friends and family and levels of importance assigned to sources of social support for relationships. They found that individuals in same-sex relationships reported less support from parents than friends and placed more value on friends (as "chosen family") compared to those in different-sex relationships. Levitt and colleagues examined alternative kinship structures in the Southern United States among African American gay and transgender adolescents and adults, finding cross-generational structures that provide support in coping with experiences of racism, heterosexism, and transphobia (Levitt, Horne, Puckett, Sweeney, \& Hampton, 2015). Frost et al. (2016) compared sources and meaning of social support 
among diverse lesbian, gay, and bisexual people in New York with heterosexual peers, finding that gay and bisexual men relied more on chosen families for major support than lesbian or bisexual women or straight people (across races). Their study revealed possible distinctions within sexual minority subcultures and the possibility that lesbians and bisexual women may be more likely to maintain and draw support from biological family compared with gay and bisexual men. A recent study by Soler and colleagues among young adult gay and bisexual men revealed that they explicitly identify chosen family members and continue to rely on these individuals for social and emotional support (Soler, Caldwell, Córdova, Harper, \& Bauermeister, 2018).

Although research on chosen families has been conducted nearly exclusively among sexual minorities, there is evidence to suggest that this queer relational form has relevance to heterosexuals as well. Muraco (2006) interviewed 46 members of 23 friendship dyads consisting of gay man/straight woman or lesbian/straight man, providing narrative data on identification as family and key functions of family in terms of financial and emotional support. She found evidence of meaning of chosen family for straight as well as gay and lesbian participants. In their community-based participatory research project with diverse youth in Chicago, Zaleski, Martin, and Messinger (2015) used multiple methods to investigate conversations about sex and sexuality with both given and chosen families. They found that youth were more comfortable discussing sex and sexuality with chosen family members (defined as "the supportive community you put together outside of the family you were given," p. 133). They also found that $80 \%$ of asexual youth reported talking with chosen family over given family, suggesting the need to educate older generations about asexuality. Youth distinguished between needs and support met by chosen (emotional, advice, intellectual) versus given (educational, financial, emotional) families. Almost $76 \%$ of youth reported "I can talk to my chosen family about anything." More than $80 \%$ reported that they had a chosen family, with support listed as the most frequent quality looked for in forming a chosen family. Many noted the chosen family as a "survival mechanism."

Similar to other normative relational forms, the idea of family and its idealized configuration has historically marginalized individuals whose desires deviate from hegemonic notions of intimacy. The model of a social unit headed by two adults of different sexes, producing biologically related offspring and viewing their unit as the primary source of social and emotional support, has been increasingly challenged for its exclusionary construction. Such a model may always have been illusory and hegemonic, even for individuals who otherwise largely conform to heteronormativity in their romantic and sexual desires (e.g., Coontz, 2016).
The limited research on chosen families as a queer relational form - an alternative construction of kinship that provides meaning, coherence, and support for individuals-reveals its historic and continued relevance. Indeed, with marriage equality achieved among sexual minorities, the construction of chosen families may assume a new intentionality (Oswald, 2002), as some resist the allure of normativity in favor of alternative kinship structures (e.g., Bannon, 2016). That is, the construction of chosen families may come to represent, for individuals of diverse sexual and gender identities and romantic orientations, a willful form of queer intimacy that may reshape larger cultural notions of family.

To interrogate the contemporary meaning of chosen families, however, researchers of sexual and gender diversity must aim to document these diverse family forms beyond what appear to be appropriations of heteronormativity (e.g., gay men parenting via donor insemination). Here, we do not claim that any one family form within queer subcultures is superior to another. Rather, we seek to draw attention to the disproportionate representation of what might be called "homonormative" forms of family within the social science literature, though we recognize that such configurations certainly are "queer" in their deviation from heteronormativity (see Pfeffer, 2017). With rare exception (e.g., Levitt et al., 2015), missing are contemporary investigations akin to that carried out by Weston (1991) or by Livingston (1991) in Paris Is Burning.

What forms of chosen family exist in the 21 st century? What social psychological processes are involved in the construction and maintenance of chosen families (Oswald, 2002)? What unique experiences related to chosen families exist for transgender and gender-nonbinary individuals? How does the meaning and construction of chosen families vary across diverse gender, race, class, and sexual identities? How have drag families (i.e., drag houses) adapted and transformed in the context of a larger culture in which gender diversity has become highly visible and a version of drag culture has become mainstream thanks to RuPaul's Drag Race? These are the kinds of questions more likely taken up by queer theorists and cultural studies scholars in the humanities. We suggest that social scientists increase their empirical attention to document queer family forms on the margins of the margins to understand the way in which diversity and creativity thrive and reveal new possibilities of human intimacy.

\section{Conclusion}

At the dawn of the scientific study of sexuality in the late 19th century, any form of same-sex sexual intimacy was severely condemned as either criminal or pathological (Foucault, 1978). By the mid-20th century, an idealized form of intimacy was codified in cultural products such as television and mass advertising as the union of two 
individuals of different sexes, married and monogamous, actively procreating and raising a social unit as a contained, nuclear family (e.g., Coontz, 2016).

While provisions of relational norms are no doubt transhistorical, the nature and meaning of relational forms shifts across time and place. Social scientists, charged with the documentation and analysis of human social and psychological life, possess the tools to describe and disseminate information about the diversity of human relationships. Our goal ought not to be the construction of knowledge that privileges some forms of intimacy over others, either explicitly or implicitly (through the failure to represent diverse forms). Rather, our goal ought to be to catalog the diversity of human intimacy and to understand the unique sources of meaning possible in relationality. In short, the norm of human relationships is diversity, and our science serves the public interest by recognizing and documenting the diverse relational forms that thrive in the 21 st century. A queer paradigm for the study of intimate diversity reveals human relationships as historically and culturally contingent sites for meaning making, promoting authenticity and social creativity.

In the 21st century, diversity in human relationships flourishes and new forms of intimacy and relational formations continue to proliferate. As a consequence, we live in an era that demands "retooling" (Kuhn, 1996) of the scientific paradigm of human relationships. Terms are in use in popular culture that are barely even cataloged by social scientists, let alone studied for their significance. Examples in our review include terms such as pansexual, graysexual, and demisexual. The discourse and meaning of human intimacy is in a perpetual state of flux, and even inherited taxonomies about gender and sexual orientation are suspect. In such a context of lived experience, we need a paradigm that is not allied with hegemonic discourses of intimacy that have historically privileged certain relational forms (e.g., heterosexuality, monogamy). Rather, we need a flexible paradigm that recognizes the diverse ways in which individuals engage with normative discourses of sex, romance, and relationships. A queer paradigm, we believe, is ideally suited for this task.

At its core, a queer paradigm rejects essentialism in all its forms and views the social world as constructed (Hostetler \& Herdt, 1998). This view need not render biology irrelevant (Fausto-Sterling, 2000, 2012; Hammack, 2005; Roughgarden, 2013; van Anders, 2015). Rather, social scientific approaches that take history and culture seriously are well equipped to consider the way in which human development is always an interaction of biology and culture, bodies and minds, and relationships, coconstructed in context (e.g., Elder, 1998; Hammack, 2005; Rogoff, 2003; Roughgarden, 2013; Shanahan, 2000). A queer paradigm privileges the link between body and mind as mediated through a rich social context (e.g., Fausto-Sterling, 2012; van Anders, 2015), itself the product of generations of intersecting narratives about the nature and meaning of human life (e.g., Fivush, Habermas, Waters, \& Zaman, 2011; Hammack, 2008; McAdams \& Guo, 2017), including narratives about gender and sexuality (Fausto-Sterling, 1985; Fine, 2010; Hammack et al., 2013). Central to this process of mediation is language (e.g., Bakhtin, 1981; Hammack \& Toolis, 2015), and the language we use about sex, gender, and intimacy creates possibilities for meaningful social practice. As social scientists, we can choose to participate in this process fully, engaging in new opportunities for "thick description" (Geertz, 1973) of the improvisations in intimacy currently under way. Our role is to document and understand, in the service of ever greater understanding of human creativity, all the while recognizing this documentation as always incomplete and ephemeral, given the open possibilities of intimate relational forms a queer paradigm acknowledges.

A new paradigm for the study of diversity in human intimacy must do more than catalog differences and similarities in different-sex and same-sex relations, in the service of acknowledging just one of countless forms of diversity. New and diverse social and political contexts call for a more expansive study of sexual and relational diversity. As we have suggested throughout this review, there are distinctions in the types of questions that need to be addressed across the various queer relational forms we have reviewed. The questions a queer paradigm provokes are all fundamentally concerned with the documentation of diversity and meaning in intimacy, so many of these questions are fundamental and descriptive in nature. In some cases, these questions have begun to be addressed in empirical research (e.g., questions of the navigation of normativity among same-sex and transgender intimacies) but require much more interrogation to represent the diversity within queer relational forms. In other cases, these questions have not been adequately addressed in empirical research (e.g., questions of the meaning and experience of intimacy among individuals who identify as graysexual; unique chosen family structures within straight communities). The questions of a queer paradigm are exponential, as they focus our lens on the documentation of diversity in a constantly shifting social landscape. The queer axiom of open possibility shifts our epistemology from one concerned with essential intelligibility of human intimacy in some transhistorical form to one fundamentally concerned with meaning in context.

A queer paradigm goes beyond the documentation of diversity, however. Its axioms reorient our fundamental assumptions about the nature and meaning of human intimacy. A queer paradigm posits that intimacy may occur among individuals of any gender identity, may change across the life course, need not be restricted to a dyadic form, may play with power and roles, need not be sexual or romantic, and may take a family form defined by intention rather than biological relation. A queer paradigm is further open to novel possibilities in a postnormative, postbinary context in which individuals are no longer constrained by 
the hegemonic assumptions of a normative paradigm of gender, sexuality, and relationships. In short, a queer paradigm upends our cultural and scientific worldview of human intimacy to better reflect the lived experience of intimate diversity in the 21 st century.

Our historical moment is characterized by the flourishing of diverse relational forms, which we see as reflective of new possibilities for the achievement of radical authenticity in human intimacy. More individuals have the opportunity to realize their desires free from the constraints of historical normativity. Their intimate projects can increasingly be codified in recognizable and legitimized vocabularies. There is a new deployment of discourse to describe human intimacy beyond the confines of the state and its institutions. Ours is thus a radically divergent historical moment from the late19th-century birth of sexology and its creation of new intimate taxonomies through the discourse of the day (Foucault, 1978). As possibilities of human connection have increased exponentially with new media environments, so too has the language of intimate desire expanded (e.g., Craig \& McInroy, 2014; Hillier \& Harrison, 2007; Saraswati, 2013).

We believe that newly visible and historically marginal forms of intimacy deserve recognition as legitimate experiences and expressions of intimacy-relationality that has meaning and value for those who practice and embody these forms. The task now for sexual and relationship science is to legitimize relational diversity through a rigorous process of documentation, oriented not toward the formation of transhistorical "truth" but rather a deep and nuanced understanding of the meaning individuals make of relationships in context. Anchored in axioms that highlight diversity and pluralism in human intimacy, a queer paradigm stands to anchor our understanding of relationships in principles that recognize and value social creativity and integrity in the expression of desire. In the process, a queer paradigm reconfigures our understanding of the meaning and possibility of human connection.

\section{Acknowledgments}

We grateful acknowledge Sari van Anders, three anonymous reviewers, and members of the Society for Personology, all of whom provided valuable feedback on earlier versions of this article.

\section{Funding}

This manuscript was completed in part while the first author was supported by a scholar award from the William T. Grant Foundation and a fellowship from the Center for Advanced Study in the Behavioral Sciences at Stanford University.

\section{References}

Adam, B. D. (2004). Care, intimacy and same-sex partnership in the 21st century. Current Sociology, 52, 265-279. doi:10.1177/0011392104041813

Adam, B. D. (2006). Relationship innovation in male couples. Sexualities, 9, 5-26. doi:10.1177/1363460706060685

Aguilar, J. (2013). Situational sexual behaviors: The ideological work of moving toward polyamory in communal living groups. Journal of Contemporary Ethnography, 42, 104-129. doi:10.1177/0891241612464886

Alegría, C. A. (2013). Relational and sexual fluidity in females partnered with male-to-female transsexual persons. Journal of Psychiatric and Mental Health Nursing, 20, 142-149. doi:10.1111/j.13652850.2011.01863.x

American Psychiatric Association. (1973). Diagnostic and statistical manual of mental disorders. Washington, D.C.: Author.

Anapol, D. M. (1997). Polyamory: The new love without limits. San Rafael, CA: IntiNet Resource Center.

Anderson, E. (2008). "Being masculine is not about who you sleep with...": Heterosexual athletes contesting masculinity and the onetime rule of homosexuality. Sex Roles, 58, 104-115. doi:10.1007/ s11199-007-9337-7

Anderson, E. (2011). The rise and fall of Western homohysteria. Journal of Feminist Scholarship, 1, 80-94.

Anderson, E., Adams, A., \& Rivers, I. (2012). "I kissed them because I love them": The emergence of heterosexual men kissing in British institutes of education. Archives of Sexual Behavior, 41, 421-430. doi:10.1007/s10508-010-9678-0

Anderson, E., \& McCormack, M. (2015). Cuddling and spooning: Heteromasculinity and homosocial tactility among student-athletes. Men and Masculinities, 18, 214-230. doi:10.1177/1097184X14523433

Anderson, E., Scoats, R., \& McCormack, M. (2015). Metropolitan bisexual men's relationships: Evidence of a cohort effect. Journal of Bisexuality, 15, 21-39. doi:10.1080/15299716.2014.994055

Austin, A., \& Goodman, R. (2017). The impact of social connectedness and internalized transphobic stigma on self-esteem among transgender and gender non-conforming adults. Journal of Homosexuality, 64, 825-841. doi:10.1080/00918369.2016.1236587

Aviram, H. (2007). Make love, not law: Perceptions of the marriage equality struggle among polyamorous activists. Journal of Bisexuality, 7, 263-286. doi:10.1080/15299710802171332

Baiocco, R., Argalia, M., \& Laghi, F. (2014). The desire to marry and attitudes toward same-sex family legalization in a sample of Italian lesbians and gay men. Journal of Family Issues, 35, 181-200. doi: $10.1177 / 0192513 \times 12464872$

Bakhtin, M. M. (1981). The dialogic imagination. (C. Emerson \& M. Holquist, Trans.). Austin, TX: University of Texas Press.

Balsam, K. F., Beauchaine, T. P., Rothblum, E. D., \& Solomon, S. E. (2008). Three-year follow-up of same-sex couples who had civil unions in Vermont, same-sex couples not in civil unions, and heterosexual married couples. Developmental Psychology, 44, 102-116. doi:10.1037/0012-1649.44.1.102

Balsam, K. F., \& Szymanski, D. M. (2005). Relationship quality and domestic violence in women's same-sex relationships: The role of minority stress. Psychology of Women Quarterly, 29, 258-269. doi:10.1111/j.1471-6402.2005.00220.x

Bannon, R. (2016, May 12). All in the family: Leather and kink families redefined. The Bay Area Reporter. Retrieved from http://www.ebar. $\mathrm{com} /$ bartab/article.php?sec $=$ leather\&article $=333$.

Barash, D. P., \& Lipton, J. E. (2001). The myth of monogamy: Fidelity and infidelity in animals and people. New York, NY: Freeman.

Barker, M. (2005). This is my partner, and this is my...partner's partner: Constructing a polyamorous identity in a monogamous world. Journal of Constructivist Psychology, 18, 75-88. doi:10.1080/ 10720530590523107

Barker, M. (2013). Consent is a grey area? A comparison of understandings of consent in Fifty Shades of Grey and on the BDSM blogosphere. Sexualities, 16, 896-914. doi:10.1177/1363460713508881 
Barker, M., \& Landridge, D. (2010). Whatever happened to non-monogamies? Critical reflections on recent research and theory. Sexualities, 13, 748-772. doi: $10.1177 / 1363460710384645$

Barker, M., \& Ritchie, A. (2007). Hot bi babes and feminist families: Polyamorous women speak out. Lesbian \& Gay Psychology Review, $8,141-151$.

Bauer, R. (2008). Transgressive and transformative gendered sexual practices and white privilege: The case of the dyke/trans/BDSM communities. Women's Studies Quarterly, 36, 233-253. doi:10.1353/ wsq. 0.0100

Bauer, R. (2018). Bois and grrrls meet their daddies and mommies on gender playgrounds: Gendered age play in the les-bi-trans-queer BDSM communities. Sexualities, 21, 139-155. doi:10.1177/ 1363460716676987

Beals, K. P., Impett, E. A., \& Peplau, L. A. (2002). Lesbians in love: Why some relationships endure and others end. Journal of Lesbian Studies, 6, 53-63. doi:10.1300/j155v06n01_06

Bell, A. P., \& Weinberg, M. S. (1978). Homosexualities: A study of diversity among men and women. New York, NY: Simon \& Schuster.

Benson, K. (2017). Tensions of subjectivity: The instability of queer polyamorous identity and community. Sexualities, 20, 24-40. doi: $10.1177 / 1363460716642154$

Berger, R. M. (1990). Men together: Understanding the gay couple. Journal of Homosexuality, 19, 31-49. doi:10.1300/j082v19n03 02

Bergstrand, C., \& Sinski, J. B. (2010). Swinging in America: Love, sex, and marriage in the 21st century. Santa Barbara, CA: Praeger.

Bettany-Saltikov, J. (2010). Learning how to undertake a systematic review: Part 1. Nursing Standard, 24, 47-55. doi:10.7748/ ns2010.08.24.50.47.c7939

Better, A. (2014). Redefining queer: Women's relationships and identity in an age of sexual fluidity. Sexuality \& Culture, 18, 16-38. doi:10.1007/s12119-013-9171-8

Bettinger, M. (2005). Polyamory and gay men: A family systems approach. Journal of GLBT Family Studies, 1, 97-116. doi:10.1300/ j461v01n01 07

Bieschke, M. (2015). Young gays are sniffing out the pup life. Vice Magazine. Retrieved from https://www.vice.com/en us/article/ mv5yw4/young-gays-are-sniffing-out-the-pup-life-979

Blackwood, E. (2000). Culture and women's sexualities. Journal of Social Issues, 56, 223-238. doi:10.1111/0022-4537.00162

Blair, K. L., \& Pukall, C. F. (2015). Family matters, but sometimes chosen family matters more: Perceived social network influence in the dating decisions of same- and mixed-sex couples. Canadian Journal of Human Sexuality, 24, 257-270. doi:10.3138/cjhs.243-a3

Blank, H. (2012). Straight: The surprisingly short history of heterosexuality. Boston, MA: Beacon.

Blasband, D., \& Peplau, L. A. (1985). Sexual exclusivity versus openness in gay male couples. Archives of Sexual Behavior, 14, 395-412. doi:10.1007/bf01542001

Blumstein, P., \& Schwartz, P. (1983). American couples. New York, NY: William Morrow \& Co.

Bogaert, A. F. (2004). Asexuality: Prevalence and associated factors in a national probability sample. Journal of Sex Research, 41, 279-287. doi:10.1080/00224490409552235

Bogaert, A. F. (2006). Toward a conceptual understanding of asexuality. Review of General Psychology, 10, 241-250. doi:10.1037/10892680.10.3.241

Bogaert, A. F. (2012). Understanding asexuality. New York, NY: Rowman \& Littlefield.

Bogaert, A. F. (2017). What asexuality tells us about sexuality. Archives of Sexual Behavior, 46, 629-630. doi:10.1007/s10508-016-0892-2

Bonello, K. (2009). Gay monogamy and extra-dyadic sex: A critical review of the theoretical and empirical literature. Counselling Psychology Review, 24, 51-65.

Bonello, K., \& Cross, M. C. (2010). Gay monogamy: I love you but I can't have sex with only you. Journal of Homosexuality, 57, 117-139. doi:10.1080/00918360903445962
Boswell, J. (1994). Same-sex unions in premodern Europe. New York, NY: Villard.

Botkin, D. R., Weeks, M. N., \& Morris, J. E. (2000). Changing marriage role expectations: 1961-1996. Sex Roles, 42, 933-942. doi:10.1023/ A: 1007006702410

Breslow, N., Evans, L., \& Langley, J. (1986). Comparisons among heterosexual, bisexual, and homosexual male sadomasochists. Journal of Homosexuality, 13, 83-107. doi:10.1300/j082v13n01 06

Bricker, M. E., \& Horne, S. E. (2007). Gay men in long-term relationships: The impact of monogamy and non-monogamy on relational health. Journal of Couple \& Relationship Therapy, 6, 27-47. doi:10.1300/j398v06n04_02

Bridges, T. (2014). A very "gay" straight? Hybrid masculinities, sexual aesthetics, and the changing relationship between masculinity and homophobia. Gender \& Society, 28, 58-82. doi:10.1177/0891243213503901

Brodsky, J. I. (1993). The Mineshaft: A retrospective ethnography. Journal of Homosexuality, 24, 233-252. doi:10.1300/j082v24n03_16

Brooks, V. R. (1981). Minority stress and lesbian women. Lanham, MD: Lexington Books.

Brotto, L. A., Knudson, G., Inskip, J., Rhodes, K., \& Erskine, Y. (2010). Asexuality: A mixed-methods approach. Archives of Sexual Behavior, 39, 599-618. doi:10.1007/s10508-008-9434-x

Butler, J. (1990). Gender trouble: Feminism and the subversion of identity. New York, NY: Routledge.

Buunk, B. P., \& van Driel, B. (1989). Alternative lifestyles and relationships. Newbury Park, CA: Sage.

Callis, A. S. (2014). Bisexual, pansexual, queer: Non-binary identities and the sexual borderlands. Sexualities, 17, 63-80. doi:10.1177/ 1363460713511094

Carrigan, M. (2011). There's more to life than sex? Difference and commonality within the asexual community. Sexualities, 14, 462478. doi: $10.1177 / 1363460711406462$

Carrillo, H., \& Hoffman, A. (2016). From MSM to heteroflexibilities: Non-exclusive straight male identities and their implications for HIV prevention and health promotion. Global Public Health, 11, 923-936. doi:10.1080/17441692.2015.1134272

Carrillo, H., \& Hoffman, A. (2018). "Straight with a pinch of bi": The construction of heterosexuality as an elastic category among adult US men. Sexualities, 21, 90-108. doi:10.1177/1363460716678561

Carrington, C. (1999). No place like home: Relationships and family life among lesbians and gay men. Chicago, IL: University of Chicago Press.

Chaline, E. R. (2010). The construction, maintenance, and evolution of gay SM sexualities and sexual identities: A preliminary description of gay SM sexual identity practices. Sexualities, 13, 338-356. doi: $10.1177 / 1363460709363323$

Chasin, C. D. (2015). Making sense in and of the asexual community: Navigating relationships and identities in a context of resistance. Journal of Community \& Applied Social Psychology, 25, 167-180. doi:10.1002/casp.2203

Chauncey, G. (1994). Gay New York: Gender, urban culture, and the making of the gay male world, 1890-1940. New York, NY: Basic Books.

Coelho, T. (2011). Hearts, groins and the intricacies of gay male open relationships: Sexual desire and liberation revisited. Sexualities, 14, 653-668. doi:10.1177/1363460711422306

Coleman, E., Rosser, B. R., \& Strapko, N. (1992). Sexual and intimacy dysfunction among homosexual men and women. Psychiatric Medicine, 10, 257-271.

Conley, T. D., \& Moors, A. C. (2014). More oxygen please! How polyamorous relationship strategies might oxygenate marriage. Psychological Inquiry, 25, 56-63. doi:10.1080/1047840x.2014.876908

Conley, T. D., Moors, A. C., Matsick, J. L., \& Ziegler, A. (2013). The fewer the merrier? Assessing stigma surrounding consensually nonmonogamous romantic relationships. Analyses of Social Issues and Public Policy, 13, 1-30. doi:10.1111/j.1530-2415.2012.01286.x 
Connell, R. W., \& Messerschmidt, J. W. (2005). Hegemonic masculinity: Rethinking the concept. Gender \& Society, 19, 829-859. doi:10.1177/ 0891243205278639

Coontz, S. (2005). Marriage, a history: From obedience to intimacy or how love conquered marriage. New York, NY: Viking.

Coontz, S. (2016). The way we never were: American families and the nostalgia trap. Revised and updated ed. New York, NY: Basic Books.

Corvino, J. (2013). What's wrong with homosexuality? New York, NY: Oxford University Press.

Cowan, T., \& LeBlanc, A. (2018). Feelings under dynamic description: The asexual spectrum and new ways of being. Journal of Theoretical and Philosophical Psychology, 38, 29-41. doi:10.1037/teo0000076

Craig, S. L., \& McInroy, L. (2014). You can form a part of yourself online: The influence of new media on identity development and coming out for LGBTQ youth. Journal of Gay \& Lesbian Mental Health, 18, 95 109. doi:10.1080/19359705.2013.777007

Crenshaw, K. (1989). Demarginalizing the intersection of race and sex: A Black feminist critique of antidiscrimination doctrine, feminist theory and antiracist politics. University of Chicago Legal Forum, 139, 139-167.

Cross, P. A., \& Matheson, K. (2006). Understanding sadomasochism: An empirical examination of four perspectives. Journal of Homosexuality, 50, 133-166. doi:10.1300/j082v50n02 07

D’Augelli, A. R., Rendina, H. J., Sinclair, K. O., \& Grossman, A. H. (2007). Lesbian and gay youth's aspirations for marriage and raising children. Journal of LGBT Issues in Counseling, 1, 77-98. doi:10.1300/j462v01n04_06

Dancer, P., Kleinplatz, P., \& Moser, C. (2006). 24/7 SM slavery. Journal of Homosexuality, 50, 81-101. doi:10.1300/j082v50n02_05

Dargie, E., Blair, K. L., Pukall, C. F., \& Coyle, S. M. (2014). Somewhere under the rainbow: Exploring the identities and experiences of trans persons. Canadian Journal of Human Sexuality, 23, 60-74. doi: $10.3138 /$ cjhs. 2378

Davidson, A. G. (1991). Looking for love in the age of AIDS: The language of gay personals, 1978-1988. Journal of Sex Research, 28 , 125-137. doi:10.1080/00224499109551598

de Lauretis, T. (1991). Queer theory: Gay and lesbian sexualities. Differences: A Journal of Feminist Cultural Studies, 3, iii-xviii.

de Visser, R., \& McDonald, D. (2007). Swings and roundabouts: Management of jealousy in heterosexual "swinging" couples. British Journal of Social Psychology, 46, 459-476. doi:10.1348/ 014466606x143153

de Vries, B., \& Megathlin, D. (2009). The meaning of friendships for gay men and lesbians in the second half of life. Journal of GLBT Family Studies, 5, 82-98. doi:10.1080/15504280802595394

Dean, J. J. (2014). Straights: Heterosexuality in post-closeted culture. New York, NY: New York University Press

DeCapua, S. R. (2017). Bisexual women's experiences with binegativity in romantic relationships. Journal of Bisexuality, 17, 451-472. doi:10.1080/15299716.2017.1382424

Degges-White, S., \& Marszalek, J. (2007). An exploration of long-term, same-sex relationships: Benchmarks, perceptions, and challenges. Journal of LGBT Issues in Counseling, 1, 99-119. doi:10.1300/ j462v01n04 07

DeLamater, J. D., \& Hyde, J. S. (1998). Essentialism vs. social constructionism in the study of human sexuality. Journal of Sex Research, 35 10-18. doi:10.1080/00224499809551913

Denfeld, D., \& Gordon, M. (1970). The sociology of mate swapping: Or the family that swings together clings together. Journal of Sex Research, 6, 85-100. doi:10.1080/00224497009550653

Denizet-Lewis, B. (2003, August 3). Living (and dying) on the down low: Double lives, AIDS and the black homosexual underground. New York Times Magazine, 28-33(48), 52-53.

Devor, H. (1993). Sexual orientation identities, attractions, and practices of female-to-male transsexuals. Journal of Sex Research, 30, 303315. doi:10.1080/00224499309551717

Diamond, L. M. (2000). Passionate friendships among adolescent sexualminority women. Journal of Research on Adolescence, 10, 191-209. doi:10.1207/SJRA1002 4
Diamond, L. M. (2002). "Having a girlfriend without knowing it": Intimate friendships among adolescent sexual-minority women. Journal of Lesbian Studies, 6, 5-16. doi:10.1300/J155v06n01_02

Diamond, L. M. (2003). What does sexual orientation orient? A biobehavioral model distinguishing romantic love and sexual desire. Psychological Review, 110, 173-192. doi:10.1037//0033-295x.110.1.173

Diamond, L. M. (2006). The intimate same-sex relationships of sexual minorities. In A. L. Vangelisti \& D. Perlman (Eds.), The Cambridge handbook of personal relationships (pp. 293-312). New York, NY: Cambridge University Press.

Diamond, L. M. (2008). Sexual fluidity: Understanding women's love and desire. Cambridge, MA: Harvard University Press.

Diamond, L. M. (2017). Three critical questions for future research on lesbian relationships. Journal of Lesbian Studies, 21, 106-119. doi:10.1080/10894160.2016.1143756

Doyle, D. M., \& Molix, L. (2015). Social stigma and sexual minorities' romantic relationship functioning: A meta-analytic review. Personality and Social Psychology Bulletin, 41, 1363-1381. doi: $10.1177 / 0146167215594592$

Easton, D., \& Hardy, J. W. (2009). The ethical slut: A practical guide to polyamory, open relationships and other adventures (2nd ed.). Berkeley, CA: Celestial Arts.

Easton, D., \& Liszt, C. A. (1997). The ethical slut: A guide to infinite sexual possibilities. San Francisco, CA: Greenery.

Elder, G. H. (1998). The life course as developmental theory. Child Development, 69, 1-12. doi:10.2307/1132065

Elizabeth, A. (2013). Challenging the binary: Sexual identity that is not duality. Journal of Bisexuality, 13, 329-337. doi:10.1080/ 15299716.2013 .813421

Ellis, H. (1925). Sexual inversion: Studies in the psychology of sex (3rd rev ed.). Philadelphia, FA: Davis.

Emens, E. F. (2004). Monogamy's law: Compulsory monogamy and polyamorous existence. New York University Review of Law and Social Change, 29, 277-376. doi:10.2139/ssrn.506242

Engel, J. W., \& Saracino, M. (1986). Love preferences and ideals: A comparison of homosexual, bisexual, and heterosexual groups. Contemporary Family Therapy, 8, 241-250. doi:10.1007/bf00902949

Evans, D. T. (2007). Sexual citizenship. Hoboken, NJ: Wiley.

Eves, A. (2004). Queer theory, butch/femme identities and lesbian space. Sexualities, 7, 480-496. doi:10.1177/1363460704047064

Faccio, E., Casini, C., \& Cipolletta, S. (2014). Forbidden games: The construction of sexuality and sexual pleasure by BDSM "players.". Culture, Health \& Sexuality, 16, 752-764. doi:10.1080/ 13691058.2014.909531

Factor, R. J., \& Rothblum, E. D. (2008). Exploring gender identity and community among three groups of transgender individuals in the United States: MTFs, FTMs, and genderqueers. Health Sociology Review, 17, 235-253. doi:10.5172/hesr.451.17.3.235

Fausto-Sterling, A. (1985). Myths of gender: Biological theories about women and men. New York, NY: Basic Books.

Fausto-Sterling, A. (2000). Sexing the body: Gender politics and the construction of sexuality. New York, NY: Basic Books.

Fausto-Sterling, A. (2012). Sex/gender: Biology in a social world. New York, NY: Routledge.

Fine, C. (2010). Delusions of gender: How our minds, society, and neurosexism create difference. New York, NY: Norton.

Finkel, E. J., Simpson, J. A., \& Eastwick, P. W. (2017). The psychology of close relationships: Fourteen core principles. Annual Review of Psychology, 68, 383-411. doi:10.1146/annurev-psych-010416044038

Fivush, R., Habermas, T., Waters, T., \& Zaman, W. (2011). The making of autobiographical memory: Intersections of culture, narratives and identity. International Journal of Psychology, 46, 321-345. doi: $10.1080 / 00207594.2011 .596541$

Flanders, C. E. (2017). Under the bisexual umbrella: Diversity of identity and experience. Journal of Bisexuality, 17, 1-6. doi:10.1080/ 15299716.2017.1297145 
Foldy, M. S. (1997). The trials of Oscar Wilde: Deviance, morality, and late-Victorian society. New Haven, CT: Yale University Press.

Foucault, M. (1978). The history of sexuality, Vol. 1: An introduction. New York, NY: Pantheon.

Frost, D., LeBlanc, A. J., de Vries, B., Alston-Stepnitz, E., Stephenson, R., \& Woodyatt, C. (2017). Couple-level minority stress: An examination of same-sex couples' unique experiences. Journal of Health and Social Behavior, 58, 455-472. doi:10.1177/0022146517736754

Frost, D. M. (2011a). Similarities and differences in the pursuit of intimacy among sexual minority and heterosexual individuals: A personal projects analysis. Journal of Social Issues, 67, 282-301. doi:10.1111/j.1540-4560.2011.01698.x

Frost, D. M. (2011b). Stigma and intimacy in same-sex relationships: A narrative approach. Journal of Family Psychology, 25, 1-10. doi: $10.1037 / \mathrm{a} 0022374$

Frost, D. M. (2014). Redemptive framings of minority stress and their association with closeness in same-sex relationships. Journal of Couple \& Relationship Therapy, 13, 219-239. doi:10.1080/ 15332691.2013.871616

Frost, D. M., \& Eliason, M. J. (2014). Challenging the assumption of fusion in female same-sex relationships. Psychology of Women Quarterly, 38, 65-74. doi:10.1177/0361684313475877

Frost, D. M., \& Fingerhut, A. W. (2016). Daily exposure to negative campaign messages decreases same-sex couples' psychological and relational well-being. Group Processes \& Intergroup Relations, 19 477-492. doi:10.1177/1368430216642028

Frost, D. M., \& Gola, K. A. (2015). Meanings of intimacy: A comparison of members of heterosexual and same-sex couples. Analyses of Social Issues and Public Policy, 15, 382-400. doi:10.1111/asap.12072

Frost, D. M., \& LeBlanc, A. J. (2014). Nonevent stress contributes to mental health disparities based on sexual orientation: Evidence from a personal projects analysis. American Journal of Orthopsychiatry, 84, 557-566. doi:10.1037/ort0000024

Frost, D. M., \& Meyer, I. H. (2009). Internalized homophobia and relationship quality among lesbians, gay men, and bisexuals. Journal of Counseling Psychology, 56, 97-109. doi:10.1037/a0012844

Frost, D. M., Meyer, I. H., \& Schwartz, S. (2016). Social support networks among diverse sexual minority populations. American Journal of Orthopsychiatry, 86, 91-102. doi:10.1037/ort0000117

Frost, D. M., \& Ouellette, S. C. (2004). Meaningful voices: How psychologists, speaking as psychologists, can inform social policy. Analyses of Social Issues and Public Policy, 4, 219-226. doi:10.1111/j.15302415.2004.00044.x

Galupo, M. P., Bauerband, L. A., Gonzalez, K. A., Hagen, D. B., Hether, S. D., \& Krum, T. E. (2014). Transgender friendship experiences: Benefits and barriers of friendships across gender identity and sexual orientation. Feminism \& Psychology, 24, 193-215. doi:10.1177/ 0959353514526218

Galupo, M. P., Davis, K. S., Grynkiewicz, A. L., \& Mitchell, R. C. (2014). Conceptualization of sexual orientation identity among sexual minorities: Patterns across sexual and gender identity. Journal of Bisexuality, 14, 433-456. doi:10.1080/15299716.2014.933466

Galupo, M. P., Henise, S. B., \& Davis, K. S. (2014). Transgender microaggressions in the context of friendship: Patterns of experience across friends' sexual orientation and gender identity. Psychology of Sexual Orientation and Gender Diversity, 1, 461-470. doi:10.1037/ $\operatorname{sgd} 0000075$

Galupo, M. P., Henise, S. B., \& Mercer, N. L. (2016). "The labels don't work very well": Transgender individuals' conceptualizations of sexual orientation and sexual identity. International Journal of Transgenderism, 17, 93-104. doi:10.1080/15532739.2016.1189373

Galupo, M. P., Krum, T. E., Hagen, D. B., Gonzalez, K. A., \& Bauerband, L. A. (2014). Disclosure of transgender identity and status in the context of friendship. Journal of LGBT Issues in Counseling, 8, 2542. doi:10.1080/15538605.2014.853638

Galupo, M. P., Mitchell, R. C., \& Davis, K. S. (2015). Sexual minority self-identification: Multiple identities and complexity. Psychology of
Sexual Orientation and Gender Diversity, 2, 355-364. doi:10.1037/ $\operatorname{sgd} 0000131$

Galupo, M. P., Pulice-Farrow, L., Clements, Z. A., \& Morris, E. R. (in press). "I love you as both and I love you as neither": Romantic partners' affirmations of nonbinary transgender individuals. International Journal of Transgenderism.

Galupo, M. P., Ramirez, J. L., \& Pulice-Farrow, L. (2017). "Regardless of their gender": Descriptions of sexual identity among bisexual, pansexual, and queer identified individuals. Journal of Bisexuality, 17, 108-124. doi:10.1080/15299716.2016.1228491

Gamarel, K. E., Reisner, S. L., Laurenceau, J., Nemoto, T., \& Operario, D. (2014). Gender minority stress, mental health, and relationship quality: A dyadic investigation of transgender women and their cisgender male partners. Journal of Family Psychology, 28, 437-447. doi:10.1037/a0037171

Gamarel, K. E., Walker, J. N. J., Rivera, L., \& Golub, S. A. (2014). Identity safety and relational health in youth spaces: A needs assessment with LGBTQ youth of color. Journal of LGBT Youth, 11, 289315. doi: $10.1080 / 19361653.2013 .879464$

Garcia, J. R., Reiber, C., Massey, S. G., \& Merriwether, A. M. (2012). Sexual hookup culture: A review. Review of General Psychology, 16, 161-176. doi:10.1037/a0027911

Geertz, C. (1973). The interpretation of cultures. New York, NY: Basic Books. Ghaziani, A. (2014). There goes the gayborhood? Princeton, NJ: Princeton University Press.

Giddens, A. (1992). The transformation of intimacy: Sexuality, love and eroticism in modern societies. Cambridge, England: Polity Press.

Goffman, E. (1959). The presentation of self in everyday life. Garden City, NY: Doubleday Anchor.

Goffman, E. (1963). Stigma: Notes on the management of spoiled identity. New York, NY: Simon \& Schuster.

Goldberg, A. E. (2013). "Doing" and "undoing" gender: The meaning and division of housework in same-sex couples. Journal of Family Theory \& Review, 5, 85-104. doi:10.1111/jftr.12009

Gonzalez, K. A., Ramirez, J. L., \& Galupo, M. P. (2017). "I was and still am": Narratives of bisexual marking in the \#StillBisexual campaign. Sexuality \& Culture, 21, 493-515. doi:10.1007/s12119-016-9401-y

Gould, S. J. (1996). The mismeasure of man (2nd ed.). New York, NY: Norton.

Green, R., \& Mitchell, V. (2002). Gay and lesbian couples in therapy: Homophobia, relational ambiguity, and social support. In A. S. Gurman (Ed.), Clinical handbook of couple therapy (3rd ed., pp. 546-568). New York, NY: Guilford.

Grunt-Mejer, K., \& Campbell, C. (2016). Around consensual nonmonogamies: Assessing attitudes toward nonexclusive relationships. Journal of Sex Research, 53, 45-53. doi:10.1080/00224499.2015.1010193

Gustavson, M. (2009). Bisexuals in relationships: Uncoupling intimacy from gender ontology. Journal of Bisexuality, 9, 407-429. doi:10.1080/15299710903316653

Hammack, P. L. (2005). The life course development of human sexual orientation: An integrative paradigm. Human Development, 48, 267290. doi: $10.1159 / 000086872$

Hammack, P. L. (2008). Narrative and the cultural psychology of identity. Personality and Social Psychology Review, 12, 222-247. doi: $10.1177 / 1088868308316892$

Hammack, P. L., Frost, D. M., Meyer, I. H., \& Pletta, D. (2018). Gay men's health and identity: Social change and the life course. Archives of Sexual Behavior, 47, 59-74. doi:10.1007/s10508-0170990-9

Hammack, P. L., Mayers, L., \& Windell, E. P. (2013). Narrative, psychology, and the politics of sexual identity in the United States: From "sickness" to "species" to "subject.". Psychology \& Sexuality, 4, 219 243. doi:10.1080/19419899.2011.621131

Hammack, P. L., \& Toolis, E. (2015). Identity, politics, and the cultural psychology of adolescence. In L. Jensen (Ed.), The Oxford handbook of human development and culture (pp. 396-409). New York, NY: Oxford University Press. 


\section{QUEER INTIMACIES}

Hardy, J. W., \& Easton, D. (2017). The ethical slut: A practical guide to polyamory, open relationships, and other freedoms in sex and love (3rd ed.). Berkeley, CA: Ten Speed Press.

Haritaworn, J., Lin, C., \& Klesse, C. (2006). Poly/logue: A critical introduction to polyamory. Sexualities, 9, 515-529. doi:10.1177/ 1363460706069963

Harrison, J., Grant, J., \& Herman, J. L. (2012). A gender not listed here: Genderqueers, gender rebels, and otherwise in the National Transgender Discrimination Survey. LGBTQ Public Policy Journal at the Harvard Kennedy School, 2. Retrieved from https://escholar ship.org/uc/item/2zj46213

Hatzenbuehler, M. L. (2009). How does sexual minority stigma "get under the skin"? A psychological mediation framework. Psychological Bulletin, 135, 707-730. doi:10.1037/a0016441

Hatzenbuehler, M. L. (2014). Structural stigma and the health of lesbian, gay, and bisexual populations. Current Directions in Psychological Science, 23, 127-132. doi:10.1177/0963721414523775

Hatzenbuehler, M. L. (2016). Structural stigma: Research evidence and implications for psychological science. American Psychologist, 71, 742-751. doi:10.1037/amp0000068

Hatzenbuehler, M. L., McLaughlin, K. A., Keyes, K. M., \& Hasin, D. S. (2010). The impact of institutional discrimination on psychiatric disorders in lesbian, gay, and bisexual populations: A prospective study. American Journal of Public Health, 100, 452-459. doi:10.2105/ajph.2009.168815

Haupert, M. L., Gesselman, A. N., Moors, A. C., Fisher, H. E., \& Garcia, J. R. (2017). Prevalence of experiences with consensual nonmonogamous relationships: Findings from two national samples of single Americans. Journal of Sex \& Marital Therapy, 43, 424-440. doi:10.1080/0092623x.2016.1178675

Hayfield, N., Clarke, V., \& Halliwell, E. (2014). Bisexual women's understandings of social marginalisation: "The heterosexuals don't understand us but nor do the lesbians.". Feminism \& Psychology, 24, 352372. doi: $10.1177 / 0959353514539651$

Herdt, G., \& Kertzner, R. (2006). I do, but I can't: The impact of marriage denial on the mental health and sexual citizenship of lesbians and gay men in the United States. Sexuality Research and Social Policy, 3, 33-49. doi:10.1525/srsp.2006.3.1.33

Herek, G. M. (1990). The context of anti-gay violence: Notes on cultural and psychological heterosexism. Journal of Interpersonal Violence, 5, 316-333. doi:10.1177/088626090005003006

Herek, G. M. (2006). Legal recognition of same-sex relationships in the United States: A social science perspective. American Psychologist, 61, 607-621. doi:10.1037/0003-066x.61.6.607

Herek, G. M. (2010). Sexual orientation differences as deficits: Science and stigma in the history of American psychology. Perspectives on Psychological Science, 5, 693-699. doi:10.1177/1745691610388770

Herek, G. M., \& Glunt, E. K. (1988). An epidemic of stigma: Public reactions to AIDS. American Psychologist, 43, 886-891. doi:10.1037//0003-066x.43.11.886

Hickson, F. C. I., Davies, P. M., Hunt, A. J., Weatherburn, P., McManus, T. J., \& Coxon, A. P. M. (1992). Maintenance of open gay relationships: Some strategies for protection against HIV. AIDS Care, 4, 409-419. doi:10.1080/09540129208253112

Hidalgo, D. A., Barber, K., \& Hunter, E. (2008). The dyadic imaginary: Troubling the perception of love as dyadic. Journal of Bisexuality, 7, 171-189. doi:10.1080/15299710802170797

Hillier, L., \& Harrison, L. (2007). Building realities less limited than their own: Young people practising same-sex attraction on the Internet. Sexualities, 10, 82-100. doi:10.1177/1363460707072956

Hines, S. (2006). Intimate transitions: Transgender practices of partnering and parenting. Sociology, 40, 353-371. doi:10.1177/0038038506062037

Hoff, C. C., \& Beougher, S. C. (2010). Sexual agreements among gay male couples. Archives of Sexual Behavior, 39, 774-787. doi:10.1007/s10508-008-9393-2

Hoff, C. C., Beougher, S. C., Chakravarty, D., Darbes, L. A., \& Neilands, T. B. (2010). Relationship characteristics and motivations behind agreements among gay male couples: Differences by agreement type and couple serostatus. AIDS Care, 22, 827-835. doi:10.1080/ 09540120903443384

Hostetler, A. J., \& Herdt, G. H. (1998). Culture, sexual lifeways, and developmental subjectivities: Rethinking sexual taxonomies. Social Research, 65, 249-290.

Hunt, M. (1974). Sexual behavior in the 1970s. Chicago, IL: Playboy Press.

Iantaffi, A., \& Bockting, W. O. (2011). Views from both sides of the bridge? Gender, sexual legitimacy and transgender people's experiences of relationships. Culture, Health \& Sexuality, 13, 355-370. doi:10.1080/13691058.2010.537770

James, E. L. (2012). Fifty shades of grey. New York, NY: Vintage.

Jenks, R. J. (1985). Swinging: A test of two theories and a proposed new model. Archives of Sexual Behavior, 14, 517-527. doi:10.1007/ bf01541752

Jenks, R. J. (1998). Swinging: A review of the literature. Archives of Sexual Behavior, 27, 507-521. doi:10.1023/A:1018708730945

Kamel, G. W. L. (1980). Leathersex: Meaningful aspects of gay sadomasochism. Deviant Behavior, 1, 171-191. doi:10.1080/01639625.1980.9967521

Katz, J. N. (2007). The invention of heterosexuality. Chicago, IL: University of Chicago Press.

Katz-Wise, S. L. (2015). Sexual fluidity in young adult women and men: Associations with sexual orientation and sexual identity development. Psychology \& Sexuality, 6, 189-208. doi:10.1080/19419899.2013.876445

Katz-Wise, S. L., \& Hyde, J. S. (2015). Sexual fluidity and related attitudes and beliefs among young adults with a same-gender orientation. Archives of Sexual Behavior, 44, 1459-1470. doi:10.1007/ s10508-014-0420-1

Katz-Wise, S. L., Reisner, S. L., Hughto, J. W., \& Keo-Meier, C. L. (2016). Differences in sexual orientation diversity and sexual fluidity in attractions among gender minority adults in Massachusetts. Journal of Sex Research, 53, 74-84. doi:10.1080/ 00224499.2014.1003028

Keleher, A., \& Smith, E. R. (2012). Growing support for gay and lesbian equality since 1990. Journal of Homosexuality, 59, 1307-1326. doi:10.1080/00918369.2012.720540

Kertzner, R. M. (2001). The adult life course and homosexual identity in midlife gay men. Annual Review of Sex Research, 12, 75-92.

Kertzner, R. M., Meyer, I. H., Frost, D. M., \& Stirratt, M. J. (2009). Social and psychological well-being in lesbians, gay men, and bisexuals: The effects of race, gender, age, and sexual identity. American Journal of Orthopsychiatry, 79, 500-510. doi:10.1037/a0016848

Kimberly, C., \& Hans, J. D. (2017). From fantasy to reality: A grounded theory of experiences in the swinging lifestyle. Archives of Sexual Behavior, 46, 789-799. doi:10.1007/s10508-015-0621-2

King, J. L. (2004). On the down low: A journey into the lives of "straight" black men who sleep with men. New York, NY: Harlem Moon.

King, M., \& Bartlett, A. (2006). What same-sex civil partnerships may mean for health. Journal of Epidemiology \& Community Health, 60, 188-191. doi:10.1136/jech.2005.040410

Kinsey, A., Pomeroy, W., \& Martin, C. (1948). Sexual behavior in the human male. Philadelphia, PA: W. B. Saunders.

Kitzinger, C., \& Wilkinson, S. (2004). Social advocacy for equal marriage: The politics of "rights" and the psychology of "mental health.". Analyses of Social Issues and Public Policy, 4, 173-194. doi:10.1111/j.1530-2415.2004.00040.x

Klesse, C. (2006). Polyamory and its "others": Contesting the terms of nonmonogamy. Sexualities, 9, 565-583. doi:10.1177/1363460706069986

Klesse, C. (2014). Polyamory: Intimate practice, identity, or sexual orientation? Sexualities, 17, 81-99. doi:10.1177/1363460713511096

Krafft-Ebing, R. (1886). Psychopathia sexualis. New York, NY: Bell.

Kuhn, T. S. (1996). The structure of scientific revolutions (3rd ed.). Chicago, IL: University of Chicago Press.

Kuper, L. E., Nussbaum, R., \& Mustanski, B. (2012). Exploring the diversity of gender and sexual orientation identities in an online sample of transgender individuals. Journal of Sex Research, 49, 244-254. doi:10.1080/00224499.2011.596954

Kurdek, L. A. (1998). Relationship outcomes and their predictors: Longitudinal evidence from heterosexual married, gay cohabiting, 
and lesbian cohabiting couples. Journal of Marriage and Family, 553-568. doi: $10.2307 / 353528$

Kurdek, L. A. (2004). Are gay and lesbian cohabiting couples really different from heterosexual married couples? Journal of Marriage and Family, 66, 880-900. doi:10.1111/j.0022-2445.2004.00060.x

Kurdek, L. A. (2005). What do we know about gay and lesbian couples? Current Directions in Psychological Science, 14, 251-254. doi:10.1111/j.0963-7214.2005.00375.x

Kurdek, L. A. (2008). A general model of relationship commitment: Evidence from same-sex partners. Personal Relationships, 15, 391405. doi:10.1111/j.1475-6811.2008.00205.x

Kurdek, L. A., \& Schmitt, J. P. (1985). Relationship quality of gay men in closed or open relationships. Journal of Homosexuality, 12, 85-99. doi: $10.1300 / \mathrm{j} 082 \mathrm{v} 12 \mathrm{n} 02 \_06$

Langdridge, D. (2006). Voices from the margins: Sadomasochism and sexual citizenship. Citizenship Studies, 10, 373-389. doi:10.1080/ 13621020600857940

Langdridge, D., \& Barker, M. (Eds.). (2007). Safe, sane, and consensual: Contemporary perspectives on sadomasochism. New York, NY: Palgrave MacMillan.

Langdridge, D., \& Butt, T. (2004). A hermeneutic phenomenological investigation of the construction of sadomasochistic identities. Sexualities, 7, 31-53. doi:10.1177/1363460704040137

Langdridge, D., \& Butt, T. (2005). The erotic construction of power exchange. Journal of Constructivist Psychology, 18, 65-73. doi:10.1080/10720530590523099

Lannutti, P. J. (2008). Attractions and obstacles while considering legally recognized same-sex marriage. Journal of GLBT Family Studies, 4, 245-264. doi:10.1080/15504280802096914

LaSala, M. (2004a). Extradyadic sex and gay male couples: Comparing monogamous and nonmonogamous relationships. Families in Society, 85, 405-412. doi:10.1606/1044-3894.1502

LaSala, M. (2004b). Monogamy of the heart: A qualitative study of extradyadic sex among gay male couples. Journal of Gay \& Lesbian Social Services, 17, 1-24. doi:10.1300/j041v17n03_01

LeBlanc, A. J., Frost, D. M., \& Wight, R. G. (2015). Minority stress and stress proliferation among same-sex and other marginalized couples. Journal of Marriage and Family, 77, 40-59. doi:10.1111/jomf.12160

Levine, M. P. (1979). Gay ghetto. Journal of Homosexuality, 4, 363-377. doi: $10.1300 /$ j082v04n04 04

Levitt, H. M., \& Horne, S. G. (2002). Explorations of lesbian-queer genders: Butch, femme, androgynous or "other". Journal of Lesbian Studies, 6, 25-39. doi:10.1300/j155v06n02_05

Levitt, H. M., Horne, S. G., Puckett, J., Sweeney, K. K., \& Hampton, M. L. (2015). Gay families: Challenging racial and sexual/gender minority stressors through social support. Journal of GLBT Family Studies, 11, 173-202. doi:10.1080/1550428X.2014.958266

Levitt, H. M., \& Ippolito, M. R. (2014). Being transgender: The experience of transgender identity development. Journal of Homosexuality, 61, 1727-1758. doi:10.1080/00918369.2014.951262

Lewis, R. J., Derlega, V. J., Griffin, J. L., \& Krowinski, A. C. (2003). Stressors for gay men and lesbians: Life stress, gay-related stress, stigma consciousness, and depressive symptoms. Journal of Social and Clinical Psychology, 22, 716-729. doi:10.1521/jscp.22.6.716.22932

Lin, K. (2017). The medicalization and demedicalization of kink: Shifting contexts of sexual politics. Sexualities, 20, 302-323. doi:10.1177/ 1363460716651420

Lindemann, D. (2011). BDSM as therapy? Sexualities, 14, 151-172. doi: $10.1177 / 1363460711399038$

Lindemann, D. J. (2010). Scripting pain: Gender, eroticism, and control in the Dominatrix's dungeon (Doctoral dissertation). New York, NY: Columbia University.

Linden, R. R. (1982). Against sadomasochism: A radical feminist analysis. East Palo Alto, CA: Frog in the Well.

Lindsey, K. (1981). Friends as family. Boston, MA: Beacon.

Livingston, J., (Producer \& Director). (1991). Paris is burning. [Motion Picture]. Los Angeles, CA: Miramax.
Macklin, E. (1980). Nontraditional family forms: A decade of research. Journal of Marriage and Family, 42, 905-922. doi:10.2307/351832

MacNeela, P., \& Murphy, A. (2015). Freedom, invisibility, and community: A qualitative study of self-identification with asexuality. Archives of Sexual Behavior, 44, 799-812. doi:10.1007/s10508-014-0458-0

Manley, M. H., Diamond, L. M., \& van Anders, S. M. (2015). Polyamory, monoamory, and sexual fluidity: A longitudinal study of identity and sexual trajectories. Psychology of Sexual Orientation and Gender Diversity, 2, 168-180. doi:10.1037/sgd0000107

Martinez, L. R., Sawyer, K. B., Thoroughgood, C. N., Ruggs, E. N., \& Smith, N. A. (2017). The importance of being "me": The relation between authentic identity expression and transgender employees' work-related attitudes and experiences. Journal of Applied Psychology, 102, 215-226. doi:10.1037/ap10000168

McAdams, D. P., \& Guo, J. (2017). The cultural shaping of life stories. In A. T. Church (Ed.), The Praeger handbook of personality across cultures (Vol. 2, pp. 185-210). Santa Barbara, CA: Praeger.

McCormack, M. (2012). The declining significance of homophobia: How teenage boys are redefining masculinity and heterosexuality. New York, NY: Oxford University Press.

McCormack, M., \& Anderson, E. (2014). The influence of declining homophobia on men's gender in the United States: An argument for the study of homohysteria. Sex Roles, 71, 109-120. doi:10.1007/ s11199-014-0358-8

McInroy, L., \& Craig, S. L. (2012). Articulating identities: Language and practice with multiethnic sexual minority youth. Counselling Psychology Quarterly, 25, 137-149. doi:10.1080/09515070.2012.674685

McLean, K. (2004). Negotiating (non)monogamy: Bisexuality and intimate relationships. Journal of Bisexuality, 4, 83-97. doi:10.1300/ j159v04n01_07

Mereish, E. H., Katz-Wise, S. L., \& Woulfe, J. (2017). We're here and we're queer: Sexual orientation and sexual fluidity differences between bisexual and queer women. Journal of Bisexuality, 17, 125-139. doi:10.1080/15299716.2016.1217448

Meyer, I. H. (1995). Minority stress and mental health in gay men. Journal of Health and Social Behavior, 36, 38-56. doi:10.2307/ 2137286

Meyer, I. H. (2003). Prejudice, social stress, and mental health in lesbian, gay, and bisexual populations: Conceptual issues and research evidence. Psychological Bulletin, 129, 674-697. doi:10.1037/00332909.129.5.674

Meyer, I. H., \& Dean, L. (1998). Internalized homophobia, intimacy, and sexual behavior among gay and bisexual men. In G. M. Herek (Ed.), Stigma and sexual orientation: Understanding prejudice against lesbians, gay men, and bisexuals (pp. 160-186). Thousand Oaks, CA: Sage. doi:10.4135/9781452243818.n8

Meyer, I. H., \& Wilson, P. A. (2009). Sampling lesbian, gay, and bisexual populations. Journal of Counseling Psychology, 56, 23-31. doi: $10.1037 / \mathrm{a} 0014587$

Mizock, L., \& Hopwood, R. (2016). Conflation and interdependence in the intersection of gender and sexuality among transgender individuals. Psychology of Sexual Orientation and Gender Diversity, 3, 93-103. doi: $10.1037 /$ sgd0000157

Mohr, J. A. (2010). Oppression by scientific method: The use of science to "other" sexual minorities. Journal of Hate Studies, 77, 21-45.

Molina, Y., Marquez, J. H., Logan, D. E., Leeson, C. J., Balsam, K. F., \& Kaysen, D. L. (2015). Current intimate relationship status, depression, and alcohol use among bisexual women: The mediating roles of bisexual-specific minority stressors. Sex Roles, 73, 43-57. doi:10.1007/s11199-015-0483-Z

Moors, A. C., Rubin, J. D., Matsick, J. L., Ziegler, A., \& Conley, T. D. (2014). It's not just a gay male thing: Sexual minority women and men are equally attracted to consensual non-monogamy. Journal Für Psychologie, 22, 1-13. doi:10.1037/e512142015-901

Morgan, E. M. (2012). Not always a straight path: College students' narratives of heterosexual identity development. Sex Roles, 66, 7993. doi:10.1007/s11199-011-0068-4 


\section{QUEER INTIMACIES}

Morgan, E. M., Steiner, M. G., \& Thompson, E. M. (2010). Processes of sexual orientation questioning among heterosexual men. Men and Masculinities, 12, 425-443. doi:10.1177/1097184x08322630

Morgan, E. M., \& Thompson, E. M. (2011). Processes of sexual orientation questioning among heterosexual women. Journal of Sex Research, 48, 16-28. doi:10.1080/00224490903370594

Moser, C., \& Levitt, E. (1987). An exploratory-descriptive study of a sadomasochistically oriented sample. Journal of Sex Research, 23, 322-337. doi:10.1080/00224498709551370

Mosher, C., Levitt, E., \& Manley, E. (2006). Layers of leather: The identity formation of leathermen as a process of transforming meanings of masculinity. Journal of Homosexuality, 51, 93-123. doi: $10.1300 / \mathrm{j} 082 \mathrm{v} 51 \mathrm{n} 03 \quad 06$

Munson, M., \& Stelbaum, J. (Eds.). (1999). The lesbian polyamory reader: Open relationships, non-monogamy, and casual sex. Philadelphia, PA: Haworth.

Muraco, A. (2006). Intentional families: Fictive kin ties between crossgender, different sexual orientation friends. Journal of Marriage and Family, 68, 1313-1325. doi:10.1111/j.1741-3737.2006.00330.x

Nardi, P. M. (1999). Gay men's friendships: Invincible communities. Chicago, IL: University of Chicago Press.

Newmahr, S. (2010). Rethinking kink: Sadomasochism as serious leisure. Qualitative Sociology, 33, 313-331. doi:10.1007/s11133-010-9158-9

Nichols, M. (2004). Lesbian sexuality/female sexuality: Rethinking "lesbian bed death". Sexual and Relationship Therapy, 19, 363-371. doi:10.1080/14681990412331298036

Normative. (2018). The Oxford English dictionary. Retrieved from http:// www.oed.com.oca.ucsc.edu/view/Entry/128294?redirectedFrom= normative\#eid

Nuttbrock, L. A., Bockting, W. O., Hwahng, S., Rosenblum, A., Mason, M., Macri, M., \& Becker, J. (2009). Gender identity affirmation among male-to-female transgender persons: A life course analysis across types of relationships and cultural/lifestyle factors. Sexual and Relationship Therapy, 24, 108-125. doi:10.1080/14681990902926764

Ordover, N. (2003). American eugenics: Race, queer anatomy, and the science of nationalism. Minneapolis, MN: University of Minnesota Press.

Ortmann, D. M., \& Sprott, R. A. (2013). Sexual outsiders: Understanding BDSM sexualities and communities. New York, NY: Rowman \& Littlefield.

Oswald, R. (2002). Resilience within the family networks of lesbians and gay men: Intentionality and redefinition. Journal of Marriage and Family, 64, 374-384. doi:10.1111/j.1741-3737.2002.00374.x

Pallotta-Chiarolli, M., \& Lubowitz, S. (2003). Outside belonging: Multisexual relationships as border existence. Journal of Bisexuality, 3, 55-85. doi:10.1300/j159v03n01_05

Parker, R. (2009). Sexuality, culture and society: Shifting paradigms in sexuality research. Culture, Health \& Sexuality, 11, 251-266. doi:10.1080/13691050701606941

Parsons, J. T., Starks, T. J., DuBois, S., Grov, C., \& Golub, S. A. (2013). Alternatives to monogamy among gay male couples in a community survey: Implications for mental health and sexual risk. Archives of Sexual Behavior, 42, 303-312. doi:10.1007/s10508-011-9885-3

Parsons, J. T., Starks, T. J., Gamarel, K. E., \& Grov, C. (2012). Non-monogamy and sexual relationship quality among same-sex male couples. Journal of Family Psychology, 26, 669-677. doi:10.1037/a0029561

Pascoe, C. J. (2005). "Dude, you're a fag": Adolescent masculinity and the fag discourse. Sexualities, 8, 329-346. doi:10.1177/1363460705053337

Pennington, S. (2009). Bisexuals "doing gender" in romantic relationships. Journal of Bisexuality, 9, 33-69. doi:10.1080/ 15299710802660029

Peplau, L. A., \& Fingerhut, A. W. (2007). The close relationships of lesbians and gay men. Annual Review of Psychology, 58, 405-424. doi:10.1146/annurev.psych.58.110405.085701

Persson, A., Newman, C. E., Manolas, P., Holt, M., Callander, D., Gordon, T., \& de Wit, J. (2017). Challenging perceptions of "straight": Heterosexual men who have sex with men and the cultural politics of sexual identity categories. Men and Masculinities. Advanced online publication. doi:10.1177/1097184x17718586
Pfeffer, C. A. (2008). Bodies in relation-Bodies in transition: Lesbian partners of trans men. Journal of Lesbian Studies, 12, 325-345. doi: $10.1080 / 10894160802278184$

Pfeffer, C. A. (2010). "Women's work"? Women partners of transgender men doing housework. Journal of Marriage and Family, 72, 165183. doi:10.1111/j.1741-3737.2009.00690.x

Pfeffer, C. A. (2012). Normative resistance and inventive pragmatism: Negotiating structure and agency in transgender families. Gender \& Society, 26, 574-602. doi:10.1177/0891243212445467

Pfeffer, C. A. (2014). "I don't like passing as a straight woman": Queer negotiations of identity and social group membership. American Journal of Sociology, 120, 1-44. doi:10.1086/677197

Pfeffer, C. A. (2017). Queering families: The postmodern partnerships of cisgender women and transgender men. New York, NY: Oxford University Press

Philpot, S. P., Duncan, D., Ellard, J., Bavinton, B. R., Grierson, J., \& Prestage, G. (2018). Negotiating gay men's relationships: How are monogamy and non-monogamy experienced and practised over time? Culture, Health \& Sexuality, 20, 915-928. doi:10.1080/ 13691058.2017.1392614

Platt, L. F., \& Bolland, K. S. (2018). Relationship partners of transgender individuals: A qualitative exploration. Journal of Social and Personal Relationships, 35, 1251-1272. doi:10.1177/0265407517709360

Plummer, K. (1995). Telling sexual stories: Power, change and social worlds. New York, NY: Routledge.

Plummer, K. (2003). Intimate citizenship: Private decisions and public dialogues. Seattle, WA: University of Washington Press.

Prause, N., \& Graham, C. A. (2007). Asexuality: Classification and characterization. Archives of Sexual Behavior, 36, 341-356. doi:10.1007/s10508-006-9142-3

Prunas, A., Fisher, A. D., Bandini, E., Maggi, M., Pace, V., Todarello, O., ... Bini, M. (2017). Eudaimonic well-being in transsexual people, before and after gender confirming surgery. Journal of Happiness Studies, 18, 1305-1317. doi:10.1007/s10902-016-9780-7

Pulice-Farrow, L., Bravo, A. P., \& Galupo, M. P. (in press). "Your gender is valid": Microaffirmations in the romantic relationships of transgender individuals. Journal of LGBT Issues in Counseling.

Reback, C. J., \& Larkins, S. (2010). Maintaining a heterosexual identity: Sexual meanings among a sample of heterosexually identified men who have sex with men. Archives of Sexual Behavior, 39, 766-773. doi:10.1007/s10508-008-9437-7

Reinhardt, R. U. (2011). Bisexual women in heterosexual relationships: A study of psychological and sociological patterns. Journal of Bisexuality, 11, 439-447. doi:10.1080/15299716.2011.620472

Reynolds, C. (2015). "I am super straight and I prefer you be too": Constructions of heterosexual masculinity in online personal ads for "straight" men seeking sex with men. Journal of Communication Inquiry, 39, 213-231. doi:10.1177/0196859915575736

Rich, A. (1980). Compulsory heterosexuality and lesbian existence. Signs, 5, 631-660. doi:10.1353/jowh.2003.0079

Richards, C. (2010). Trans and non-monogamies. In M. Barker \& D. Langdridge (Eds.), Understanding non-monogamies (pp. 121-133). New York, NY: Routledge. doi:10.4324/9780203869802

Richards, C., Bouman, W. P., Seal, L., Barker, M. J., Nieder, T. O., \& T'Sjoen, G. (2016). Non-binary or genderqueer genders. International Review of Psychiatry, 28, 95-102. doi:10.3109/ 09540261.2015 .1106446

Richters, J., de Visser, R. O., Rissel, C. E., Grulich, A. E., \& Smith, A. M. A. (2008). Demographic and psychosocial features of participants in bondage and discipline, "sadomasochism" or dominance and submission (BDSM): Data from a national survey. Journal of Sexual Medicine, 5, 1660-1668. doi:10.1111/j.1743-6109.2008.00795.x

Ridinger, R. (2002). Things visible and invisible: The Leather Archives and Museum. Journal of Homosexuality, 43, 1-9. doi:10.1300/ j082v43n01 01

Ridinger, R. (2006). Negotiating limits: The legal status of SM in the United States. Journal of Homosexuality, 50, 189-216. doi:10.1300/ j082v50n02 09 
Riggle, E. D., Thomas, J. D., \& Rostosky, S. S. (2005). The marriage debate and minority stress. PS: Political Science \& Politics, 38, $221-$ 224. doi:10.1017/S1049096505056337

Riggle, E. D., Whitman, J. S., Olson, A., Rostosky, S. S., \& Strong, S. (2008). The positive aspects of being a lesbian or gay man. Professional Psychology: Research and Practice, 39, 210-217. doi: $10.1037 / 0735-7028.39 .2 .210$

Ritchie, A., \& Barker, M. (2006). "There aren't words for what we do or how we feel so we have to make them up": Constructing polyamorous languages in a culture of compulsory monogamy. Sexualities, 9, 584-601. doi:10.1177/1363460706069987

Robbins, N. K., Low, K. G., \& Query, A. N. (2016). A qualitative exploration of the "coming out" process for asexual individuals. Archives of Sexual Behavior, 45, 751-760. doi:10.1007/s10508-015-0561-x

Robinson, B. A., \& Vidal-Ortiz, S. (2013). Displacing the dominant "down low" discourse: Deviance, same-sex desire, and Craigslist.org. Deviant Behavior, 34, 224-241. doi:10.1080/01639625.2012.726174

Robinson, M. (2013). Polyamory and monogamy as strategic identities. Journal of Bisexuality, 13, 21-38. doi:10.1080/15299716.2013.755731

Rogoff, B. (2003). The cultural nature of human development. New York, NY: Oxford University Press.

Rostosky, S. S., \& Riggle, E. D. (2017a). Same-sex couple relationship strengths. Psychology of Sexual Orientation and Gender Diversity, 4 1-13. doi: $10.1037 / \mathrm{sgd} 0000216$

Rostosky, S. S., \& Riggle, E. D. (2017b). Same-sex relationships and minority stress. Current Opinion in Psychology, 13, 29-38. doi: $10.1037 /$ sgd0000216

Rostosky, S. S., Riggle, E. D., Rothblum, E. D., \& Balsam, K. F. (2016). Same-sex couples' decisions and experiences of marriage in the context of minority stress: Interviews from a population-based longitudinal study. Journal of Homosexuality, 63, 1019-1040. doi:10.1080/00918369.2016.1191232

Rostosky, S. S., Riggle, E. D. B., Gray, B. E., \& Hatton, R. L. (2007). Minority stress experiences in committed same-sex couple relationships. Professional Psychology: Research and Practice, 38, 392-400. doi:10.1037/0735-7028.38.4.392

Rothblum, E. D. (2005). Same-sex marriage and legalized relationships: I do, or do I? Journal of GLBT Family Studies, 1, 21-31. doi:10.1300/ j461v01n01_03

Rothblum, E. D. (2006). From science fiction to computer-generated technology: Sampling lesbian, gay, and bisexual individuals. In I. H. Meyer \& M. E. Northridge (Eds.), The health of sexual minorities: Public health perspectives on lesbian, gay, bisexual and transgender populations (pp. 442-454). New York, NY: Springer. doi:10.1007/ 978-0-387-31334-4 17

Rothblum, E. D., Balsam, K. F., \& Wickham, R. E. (2018). Butch, femme, and androgynous gender identities within female same-sex couples: An actor-partner analysis. Psychology of Sexual Orientation and Gender Diversity, 5, 72-81. doi:10.1037/sgd0000258

Rothblum, E. D., \& Brehony, K. A. (Eds). (1993). Boston marriages. Romantic but asexual relationships among contemporary lesbians. Amherst, MA: University of Massachusetts Press.

Roughgarden, J. (2013). Evolution's rainbow: Diversity, gender, and sexuality in nature and people (10th anniversary ed.). Berkeley, CA: University of California Press.

Rubel, A. N., \& Bogaert, A. F. (2015). Consensual nonmonogamy: Psychological well-being and relationship quality correlates. Journal of Sex Research, 52, 961-982. doi:10.1080/ 00224499.2014.942722

Rubin, G. S. (2011a). The Catacombs: A temple of the butthole. In G. S. Rubin (Ed.), Deviations: A Gayle Rubin reader (pp. 224-240). Durham, NC: Duke University Press. (Original work published 1991).

Rubin, G. S. (2011b). The leather menace: Comments on politics and S/ M. In G. S. Rubin (Ed.), Deviations: A Gay Rubin reader (pp. 109-136). Durham, NC: Duke University Press. (Original work published 1981)

Rubin, G. S. (2011c). Thinking sex: Notes for a radical theory of the politics of sexuality. In G. S. Rubin (Ed.), Deviations: A Gayle Rubin reader (pp. 137-181). Durham, NC: Duke University Press. (Original work published 1984). doi:10.1215/9780822394068-006

Russell, G. M., \& Richards, J. A. (2003). Stressor and resilience factors for lesbians, gay men, and bisexuals confronting antigay politics. American Journal of Community Psychology, 31, 313-328. doi:10.1023/a:1023919022811

Rust, P. C. R. (2002). Bisexuality: The state of the union. Annual Review of Sex Research, 13, 180-240. doi:10.1080/ 10532528.2002.10559805

Sadownick, D. (1996). Sex between men: An intimate history of the sex lives of gay men postwar to present. San Francisco, CA: Harper San Francisco.

Sanger, T. (2010). Trans people's partnerships: Towards an ethics of intimacy. New York, NY: Palgrave Macmillan.

Saraswati, L. A. (2013). Wikisexuality: Rethinking sexuality in cyberspace. Sexualities, 16, 587-603. doi:10.1177/1363460713487368

Savin-Williams, R. C. (2017). Mostly straight: Sexual fluidity among men. Cambridge, MA: Harvard University Press.

Savin-Williams, R. C. (2018). An exploratory study of exclusively heterosexual, primarily heterosexual, and mostly heterosexual young men. Sexualities, 21, 16-29. doi:10.1177/1363460716678559

Savin-Williams, R. C., \& Vrangalova, Z. (2013). Mostly heterosexual as a distinct sexual orientation group: A systematic review of the empirical evidence. Developmental Review, 33, 58-88. doi:10.1016/j. dr 2013.01.001

Scherrer, K. S. (2008). Coming to an asexual identity: Negotiating identity, negotiating desire. Sexualities, 11, 621-641. doi:10.1177/ 1363460708094269

Schimmel-Bristow, A., Haley, S. G., Crouch, J. M., Evans, Y. N., Ahrens, K. R., McCarty, C. A., \& Inwards-Breland, D. J. (2018). Youth and caregiver experiences of gender identity transition: A qualitative study. Psychology of Sexual Orientation and Gender Diversity, 5, 273-281. doi:10.1037/sgd0000269

Scott, S., \& Dawson, M. (2015). Rethinking asexuality: A symbolic interactionist account. Sexualities, 18, 3-19. doi:10.1177/1363460714531273

Sedgwick, E. K. (1990). Epistemology of the closet. Berkeley, CA: University of California Press.

Seidman, S. (Ed.). (1996). Queer theory/sociology. Malden, MA: Blackwell.

Seidman, S. (2015). The social construction of sexuality (3rd ed.). New York, NY: Norton.

Serano, J. (2007). Whipping girl: A transsexual woman on sexism and the scapegoating of femininity. Emeryville, CA: Seal Press.

Shanahan, M. J. (2000). Pathways to adulthood in changing societies: Variability and mechanisms in life course perspective. Annual Review of Sociology, 26, 667-692. doi:10.1146/annurev. soc.26.1.667

Sheff, E. (2005). Polyamorous women, sexual subjectivity and power. Journal of Contemporary Ethnography, 34, 251-283. doi:10.1177/ 0891241604274263

Sheff, E. (2006). Poly-hegemonic masculinities. Sexualities, 9, 621-642. doi: $10.1177 / 1363460706070004$

Sheff, E. (2011). Polyamorous families, same-sex marriage, and the slippery slope. Journal of Contemporary Ethnography, 40, 487-520. doi: $10.1177 / 0891241611413578$

Sheff, E., \& Hammers, C. (2011). The privilege of perversities: Race, class and education among polyamorists and kinksters. Psychology \& Sexuality, 2, 198-223. doi:10.1080/19419899.2010.537674

Silva, T. (2017). Bud-sex: Constructing normative masculinity among rural straight men that have sex with men. Gender \& Society, 31, 51-73. doi:10.1177/0891243216679934

Silva, T. J. (2018). "Helpin' a buddy out": Perceptions of identity and behaviour among rural straight men that have sex with each other. Sexualities, 21, 68-89. doi:10.1177/1363460716678564

Sloan, L. J. (2015). Ace of (BDSM) clubs: Building asexual relationships through BDSM practice. Sexualities, 18, 548-563. doi:10.1177/ 1363460714550907

Smith, J. R., \& Smith, L. G. (1970). Co-marital sex and the sexual freedom movement. Journal of Sex Research, 6, 131-142. doi:10.1080/ 00224497009550656 


\section{QUEER INTIMACIES}

Soler, J. H., Caldwell, C. H., Córdova, D., Harper, G., \& Bauermeister, J. A. (2018). Who counts as family? Family typologies, family support, and family undermining among young adult gay and bisexual men. Sexuality Research and Social Policy, 15, 123-138. doi:10.1007/ s13178-017-0288-7

Stacey, J. (2005). The families of man: Gay male intimacy and kinship in a global metropolis. Signs, 30, 1911-1935. doi:10.1086/426797

Sternberg, R. J. (1986). A triangular theory of love. Psychological Review, 93, 119-135. doi:10.4135/9781412956253.n591

Stiles, B. L., \& Clark, R. E. (2011). BDSM: A subcultural analysis of sacrifices and delights. Deviant Behavior, 32, 158-189. doi:10.1080/ 01639621003748605

Szymanski, D. M., \& Mikorski, R. (2016). External and internalized heterosexism, meaning in life, and psychological distress. Psychology of Sexual Orientation and Gender Diversity, 3, 265274. doi: $10.1037 / \mathrm{sgd} 0000182$

Tate, C. C., Youssef, C. P., \& Bettergarcia, J. N. (2014). Integrating the study of transgender spectrum and cisgender experiences of selfcategorization from a personality perspective. Review of General Psychology, 18, 302-312. doi:10.1037/gpr0000019

Taylor, G., \& Ussher, J. (2001). Making sense of S\&M: A discourse analytic account. Sexualities, 4, 293-314. doi:10.1177/136346001004003002

Teich, N. M. (2012). Transgender 101: A simple guide to a complex issue. New York, NY: Columbia University Press.

Thompson, E. M. (2007). Girl friend or girlfriend? Same-sex friendship and bisexual images as a context for flexible sexual identity among young women. Journal of Bisexuality, 6, 47-67. doi:10.1300/ $\mathrm{J} 159 \mathrm{v} 06 \mathrm{n} 03$ 04

Thompson, E. M., \& Morgan, E. M. (2008). "Mostly straight" young women: Variations in sexual behavior and identity development. Developmental Psychology, 44, 15-21. doi:10.1037/00121649.44.1.15

Timmins, L., Rimes, K. A., \& Rahman, Q. (2017). Minority stressors and psychological distress in transgender individuals. Psychology of Sexual Orientation and Gender Diversity, 4, 328-340. doi:10.1037/ sgd0000237.supp

Traies, J. (2015). Old lesbians in the UK: Community and friendship. Journal of Lesbian Studies, 19, 35-49. doi:10.1080/ 10894160.2015.959872

van Anders, S. M. (2015). Beyond sexual orientation: Integrating gender/ sex and diverse sexualities via Sexual Configurations Theory. Archives of Sexual Behavior, 44, 1177-1213. doi:10.1007/s10508015-0490-8

van Eeden-Moorefield, B., Malloy, K., \& Benson, K. (2016). Gay men's (non) monogamy ideals and lived experience. Sex Roles, 75, 43-55. doi:10.1007/s11199-015-0566-x

van Eeden-Moorefield, B., Martell, C. R., Williams, M., \& Preston, M. (2011). Same-sex relationships and dissolution: The connection between heteronormativity and homonormativity. Family Relations, 60, 562-571. doi:10.1111/j.1741-3729.2011.00669.x

van Hooff, J. (2013). Modern couples? Continuity and change in heterosexual relationships. New York, NY: Routledge.

van Houdenhove, E., Gijs, L., T'Sjoen, G., \& Enzlin, P. (2015). Asexuality: A multidimensional approach. Journal of Sex Research, 52, 669-678. doi:10.1080/00224499.2014.898015

Velez, B. L., Breslow, A. S., Brewster, M. E., Cox, R., \& Foster, A. B. (2016). Building a pantheoretical model of dehumanization with transgender men: Integrating objectification and minority stress theories. Journal of Counseling Psychology, 63, 497-508. doi:10.1037/cou0000136

Vrangalova, Z., \& Savin-Williams, R. C. (2012). Mostly heterosexual and mostly gay/lesbian: Evidence for new sexual orientation identities. Archives of Sexual Behavior, 41, 85-101. doi:10.1007/s10508-0129921-y

Ward, J. (2008). Dude-sex: White masculinities and "authentic" heterosexuality among dudes who have sex with dudes. Sexualities, 11, 414-434. doi:10.1177/1363460708091742
Ward, J. (2010). Gender labor: Transmen, femmes, and the collective work of transgression. Sexualities, 13, 236-254. doi:10.1177/ 1363460709359114

Ward, J. (2015). Not gay: Sex between straight white men. New York, NY: New York University Press.

Warner, M. (1999). The trouble with normal: Sex, politics, and the ethics of queer life. Cambridge, MA: Harvard University Press.

Weeks, J., Heaphy, B., \& Donovan, C. (2001). Same-sex intimacies: Families of choice and other life experiments. London, UK: Routledge.

Weinberg, M. S., Williams, C. J., \& Pryor, D. W. (1994). Dual attraction: Understanding bisexuality. New York, NY: Oxford University Press.

Weinberg, T. S. (1987). Sadomasochism in the United States: A review of recent sociological literature. Journal of Sex Research, 23, 50-69. doi:10.1080/00224498709551341

Weinberg, T. S. (1994). Research in sadomasochism: A review of sociological and social psychological literature. Annual Review of Sex Research, 5, 257-279. doi:10.1080/10532528.1994.10559898

Weinberg, T. S. (2006). Sadomasochism and the social sciences: A review of the sociological and social psychological literature. Journal of Homosexuality, 50, 17-40. doi:10.1300/j082v50n02_02

Weinberg, T. S., \& Falk, G. (1980). The social organization of sadism and masochism. Deviant Behavior, 1, 379-393. doi:10.1080/ 01639625.1980.9967534

Weinberg, T. S., Williams, C. J., \& Moser, C. (1984). The social constituents of sadomasochism. Social Problems, 31, 379-393. doi: $10.2307 / 800385$

Weinstock, J. S. (2000). Lesbian friendships at midlife: Patterns and possibilities for the 21st century. Journal of Gay \& Lesbian Social Services, 11, 1-32. doi:10.1300/j041v11n02 01

Weiss, J. T. (2003). GL vs. BT: The archeology of biphobia and transphobia within the U.S. gay and lesbian community. Journal of Bisexuality, 3, 25-55. doi:10.1300/j159v03n03_02

Weiss, M. D. (2006). Working at play: BDSM sexuality in the San Francisco Bay area. Anthropologica, 8, 229-245. doi:10.2307/25605313

Wernick, L. J., Kulick, A., \& Chin, M. (2017). Gender identity disparities in bathroom safety and wellbeing among high school students. Journal of Youth and Adolescence, 46, 917-930. doi:10.1007/ s10964-017-0652-1

Weston, K. (1991). Families we choose: Lesbians, gays, kinship. New York, NY: Columbia University Press.

Wignall, L., \& McCormack, M. (2017). An exploratory study of a new kink activity: "Pup play.". Archives of Sexual Behavior, 26, 801-811. doi:10.1007/s10508-015-0636-8

Williams, S. L., \& Mann, A. K. (2017). Sexual and gender minority health disparities as a social issue: How stigma and intergroup relations can explain and reduce health disparities. Journal of Social Issues, 73, 450-461. doi:10.1111/josi.12236

Worth, H., Reid, A., \& McMillan, K. (2002). Somewhere over the rainbow: Love, trust, and monogamy in gay relationships. Journal of Sociology, 38, 237-253. doi:10.1177/144078302128756642

Wosick-Correa, K. (2010). Agreements, rules, and agentic fidelity in polyamorous relationships. Psychology \& Sexuality, 1, 44-61. doi:10.1080/19419891003634471

Wright, S. (2006). Discrimination of SM-identified individuals. Journal of Homosexuality, 50, 217-231. doi:10.1300/j082v50n02_10

Yule, M. A., Brotto, L. A., \& Gorzalka, B. B. (2014). Biological markers of asexuality: Handedness, birth order, and finger length ratios in selfidentified asexual men and women. Archives of Sexual Behavior, 43, 299-310. doi:10.1007/s10508-013-0175-0

Zaleski, N., Martin, P., \& Messinger, J. (2015). Given and chosen: Youth-led research on family-supported conversations about sexuality. Family \& Community Health: the Journal of Health Promotion \& Maintenance, 38, 120-130. doi:10.1097/ fch.0000000000000051 